Modified Airy Function and WKB Solutions to the Wave Equation

A. K. Ghatak, R. L. Gallawa, and I. C. Goyal

$-Q C$ 100 .0556 \#176 



\title{
Modified Airy Function and WKB Solutions to the Wave Equation
}

\author{
A. K. Ghatak \\ R. L. Gallawa \\ I. C. Goyal
}

Elcctromagnetic Technology Division

Elcctronics and Electrical Engineering Laboratory

National Institute of Standards and Technology

Boulder, CO 80303

This monograph was prepared, in part, under the auspices of the Indo-US Collaborative Program in Materials Sciences.

Pcrmanent Affiliation of Professors Ghatak and Goyal is

Physics Department, Indian Institute of Technology

Ncw Delhi, India.

November 1991

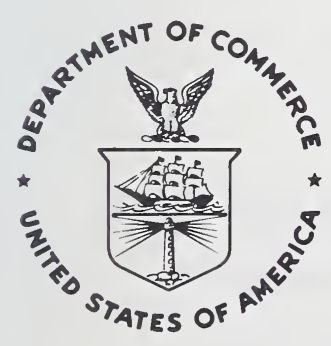

U.S. Department of Commerce

Robert A. Mosbacher, Secretary

National Institute of Standards and Technology John W. Lyons, Director 
National Institute of Standards and Technology Monograph 176

Natl. Inst. Stand. Technol. Mono. 176, 172 pages (Nov. 1991)

CODEN: NIMOEZ

\section{U.S. GOVERNMENT PRINTING OFFICE}

WASHINGTON: 1991

For sale by the Superintendent of Documents, U.S. Government Printing Office, Washington, DC 20402-9325 


\section{TABLE OF CONTENTS}

PREFACE $\ldots \ldots \ldots \ldots \ldots \ldots \ldots$ vii

1. INTRODUCTION $\ldots \ldots \ldots \ldots \ldots \ldots$

2. WKB SOLUTIONS TO INITIAL VALUE PROBLEMS . . . . . . . . . . . . 6

2.1 Introduction $\ldots \ldots \ldots \ldots \ldots \ldots$

2.2 The WKB Solutions . . . . . . . . . 6

2.3 An Alternative Derivation . . . . . . . . . . 11

2.4 The General WKB Solution . . . . . . . . . . 14

Case I: Barrier to the Right . . . . . . . . 15

Case II: Barrier to the Left . . . . . . . . . . 19

2.5 Examples . . . . . . . . . . . . . . 21

Example $2.1 \ldots \ldots \ldots \ldots \ldots \ldots . \ldots 21$

Example $2.2 \ldots \ldots \ldots \ldots \ldots \ldots$

3. AIRY FUNCTIONS . . . . . . . . . . . 32

3.1 Introduction $\ldots \ldots \ldots \ldots \ldots \ldots \ldots \ldots$

3.2 The Airy Functions . . . . . . . . . . . . . 32

3.3 Asymptotic Forms and the Connection Formulas . . . . . . . . . . . . 37

4. MAF SOLUTION TO INITIAL VALUE PROBLEMS . . . . . . . . . . . . . . 42

4.1 Introduction $\ldots \ldots \ldots \ldots \ldots \ldots \ldots$ 
4.2 The $M A F$ Solution ............ 43

4.3 Examples .................... 47

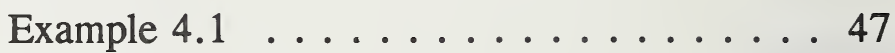

4.4 Summary ..................... 49

5. WKB SOLUTIONS TO EIGENVALUE

PROBLEMS ....................... 53

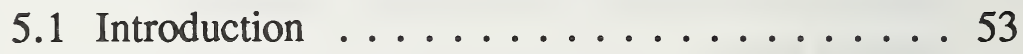

5.2 The WKB Quantization Condition ...... 59

5.3 Examples ................... 63

Example $5.1 \ldots \ldots 63$

Example $5.2 \ldots \ldots 69$

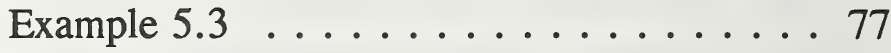

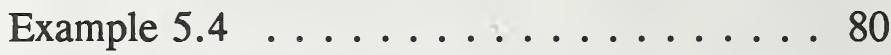

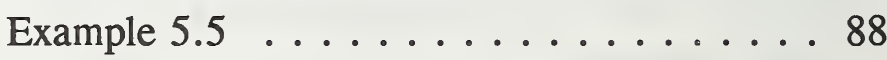

5.4 The WKB Method for Three-Dimensional

Problems ................... 92

Example $5.6 \ldots \ldots . \ldots . \ldots 94$

6. MAF SOLUTIONS TO EIGENVALUE

PROBLEMS ........................ 97

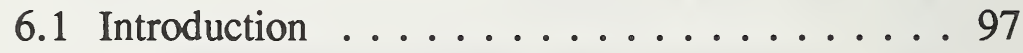

6.2 Formulation of the Problem ......... 98

6.3 Examples ................... 100

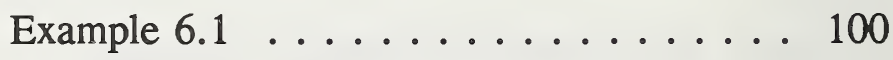

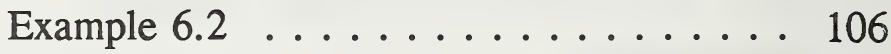

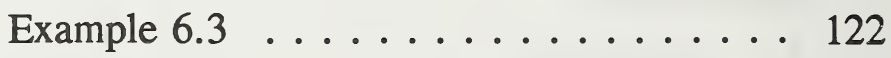

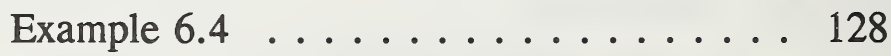

Example $6.5 \ldots \ldots . \ldots . \ldots 135$ 
6.4 Conclusions . . . . . . . . . . . . 140

7. EXTENSION OF MAF METHOD FOR EIGENVALUES . . . . . . . . . . . . . 143

7.1 Introduction $\ldots \ldots \ldots \ldots \ldots \ldots \ldots . \ldots . \ldots . \ldots$

7.2 Formulation and Example $7.1 \ldots \ldots . . .143$

Example $7.2 \ldots \ldots \ldots \ldots . . . \ldots 149$

Example $7.3 \ldots \ldots \ldots \ldots \ldots \ldots$

7.3 Conclusions $\ldots \ldots \ldots \ldots \ldots \ldots \ldots$ 



\section{PREFACE}

The WKB method has been known and used by students of mathematical physics since the mid-1920s. It has served amazingly well in a variety of problems, especially in problems of quantum mechanics and nonuniform optical waveguides, the discipline treated in this monograph. The success and popularity of the method occurs in spite of a serious deficiency, namely that it does not hold at the turning points unless suitable modifications are invoked. It is, at once, intuitive and powerful, and it sometimes yields very accurate eigenvalues in spite of its inherent approximate nature. Its use by the optics community is well documented. The real power of the method lies in its utility; it is useful in an amazing array of physics problems.

The WKB method springs from a knowledge of the solution to the governing differential equation when the nonuniform refractive index (for waveguide problems) or potential function (for quantum well problems) is uniform. The resulting method yields the exact solution if the nonuniform region becomes uniform. In an analogous manner, this monograph concentrates on a method that becomes exact if the nonuniform region takes on a linear variation. The method is thus based on the solution to the governing equation when the nonuniform region varies linearly with $x$; this suggests that the result is a higher order approximation than the WKB approximation. Numerical tests indicate that this seems to be the case. In addition, and in contrast to the WKB method, these equations have the significant advantage of being valid even in the 
vicinity of and at the turning point. For problems of interest in optical telecommunications devices, there is, in fact, a turning point, at which there is a first order zero. It is this class of problems that is discussed in this monograph.

What we present in this monograph uses a modification of the Airy functions to describe the wave functions; we have, therefore, chosen to refer to the method as the Modified Airy Function or $M A F$ method. The method used here is not new. It was developed 60 years ago in a classic paper by Langer (1931), in which he developed an asymptotic solution to the Helmholtz equation. The $M A F$ equations can be recast in the form used by Langer. Langer used Bessel functions; we choose to use Airy functions. The two are equivalent. In working with Langer's equations, we have found, to our dismay, that the method has been dramatically underutilized by the scientific community. It is, therefore, appropriate to show, through illustrative examples, the power, the simplicity, and the profound utility of the $M A F$ method. Herein lies the purpose of this monograph.

This monograph discusses the $W K B$ and the $M A F$ methods in considerable detail, to the end that the reader gains an appreciation of the strengths and weaknesses of each. We treat eigenvalue problems as well as initial value problems. The two methods could be illustrated via any of several disciplines, but we chose to use problems that are of current interest to the optical waveguide community. We have also used Schrödinger's equation for typical potential well problems to further illustrate the method. Both the wave functions and the eigenvalues are found. We have also 
included several exercises, to be solved by the reader. We trust and hope that, by giving several examples to illustrate the method, the reader will appreciate the utility of the method.

Advancements in the understanding of physical science often spring from the harmonic convergence of fortuitous but unpredictable (sometimes serendipitous) events. Techniques in mathematical physics, being intimately related to the physical sciences, follow a similar trend. The fortuitous events that led, ultimately, to the writing of this monograph are traceable to the vision, encouragement, and dynamic leadership of colleagues and friends to whom we are profoundly indebted. They include (in alphabetical order) Dr. B. Stephen Carpenter (NIST Director of International Relations), Dr. P.L.M. Heydemann (Science Counselor, U.S. Embassy, New Delhi), Dr. R.A. Kamper (NIST, Boulder), Professor N.C. Nigam (Director, IIT New Delhi), and Dr. Alfons Weber (NIST, Gaithersburg). Their encouragement and support (both financial and moral) is deeply appreciated. Finally, a special thanks to Mr. Aaron A. Sanders (NIST, Boulder), whose enthusiastic support was pivotal to the writing of this monograph. 



\section{INTRODUCTION}

The purpose of this monograph is to revisit a basic equation of mathematical physics,

$$
\frac{d^{2} \psi}{d x^{2}}+\Gamma^{2}(x) \psi(x)=0,
$$

and to give approximate solutions based on the WKB method and on a modification of the Airy function. All of the examples that we will use to illustrate the methods are based on optical waveguides and quantum mechanical problems. We hope that this monograph will prove to be tutorial, giving insight and understanding to the use of Airy functions in addressing the scalarwave equation.

Equation (1) is encountered in many areas of physics and engineering. In quantum mechanics, for example, the onedimensional Schrödinger equation is of the same form as Eq. (1) with

$$
\Gamma^{2}(x)=\frac{2 m}{\hbar^{2}}[E-V(x)],
$$

where $m$ is the mass of the particle, $E$ the total energy, $V(x)$ the potential energy function and $\hbar \equiv h / 2 \pi$, h being Planck's constant. In Eq. (2) $E$ appears as an eigenvalue in a bound state problem and 
as a given (variable) parameter in a scattering problem [see, e.g., Schiff (1968)]. Similarly, in optical waveguide theory, for a medium characterized by the refractive index variation $n^{2}(x)$, the $y$-component of the electric field can be written in the form

$$
E_{y}(x, z, t)=\psi(x) \exp [i(\omega t-\beta z)]
$$

where $\Psi(x)$ satisfies Eq. (1) with

$$
\Gamma^{2}(x)=k_{o}^{2} n^{2}(x)-\beta^{2}
$$

and $k_{0}=\omega / c$ is the free space wave number. Equation (3) describes the TE modes of the slab waveguide. In Eqs. (3) and (4), $\beta$ is the propagation constant which forms a discrete set for guided modes and a continuum for iadiation modes [see, e.g., Ghatak and Thyagarajan (1989)].

For some specific profiles, that is, for some specific forms of $\Gamma^{2}$ $(x)$, we can obtain analytical solutions of Eq. (1). However, for profiles which do not lead to exact solutions, we usually resort to one of the three approximate methods: the perturbation method, the variational method, and the $W K B$ method. The perturbation method is based on a closely related problem which yields an exact solution and we usually resort to first-order perturbation; even then it is extremely difficult to calculate the perturbed eigenfunction as it would involve the summation over an infinite series. On the other hand, the variational method can give a good estimate for the lowest-order mode by choosing an appropriate trial function and carrying out an optimization; the method becomes quite cumbersome when one has to apply it to higher-order modes. 
The WKB method was first proposed in the mid-1920s by Wentzel (1926), Kramers (1926), Brillouin (1926), and Jeffreys (1923); indeed because of the contributions made by Jeffreys, the method is often referred to as the WKBJ or even JWKB method.

The traditional WKB method is well known and has been used extensively [Fröman and Fröman (1965), Heading (1962), Pauli (1980), or almost any text on quantum mechanics]. While several improvements have been suggested [see Hecht (1957), Bahar (1967)], the method remains primarily the tool that was originally proposed.

Our intent is to be primarily tutorial, discussing both the traditional $W K B$ method and a relatively unknown competing and much more powerful technique that uses Airy functions. This method is WKBlike and was first suggested by Langer (1931). The advantages and disadvantages of each method will be demonstrated by way of examples.

Langer's 1931 paper is pivotal to what is presented here. In our introduction to that method, we will give a derivation that is less esoteric than that of Langer. We will illustrate the method with examples, in the hope that the method will be more widely known and more widely used. We note in this regard that the Modified Airy Function method (or $M A F$, which is how we will refer to the method formulated by Langer) is not well known and is seldom used in modern mathematical physics. The analysis of nonuniform optical waveguides (eigenvalue problems), for example, benefits greatly from this approach, a fact that will be illustrated. Initial- 
value problems are also amenable to the $M A F$ method.

There is a profound and glaring paucity of literature on the $M A F$ method, in spite of its importance, its range of applications, and its accuracy. It is, happily, quite easy to use, even with a desktop computer; the ease is attributable to the ease with which Airy functions (the basis of the solution) can be evaluated.

The framework of the MAF solution appears in a few books but the method is not exploited. An important exception is the book by Bender and Orszag (1978), where the method is described in a very understandable form. What we present in this monograph is a supplement to the presentation of Bender and Orszag. We use current examples to illustrate the method.

Because the MAF solutions use Airy functions, it may be thought that they are the traditional $W K B$ result, wherein the Airy functions are used to derive the connection formulas. However, the MAF solutions give, in a single closed form, an approximation that holds throughout the region of interest, including (but not restricted to) the vicinity of and at a turning point; a turning point is where $\Gamma^{2}(x)$ becomes zero. The WKB solutions are valid in regions far removed from the turning point, but not in its vicinity.

While solving the eigenvalue problem we will show that use of the first-order perturbation theory, using the present Modified Airy Function solutions, yields extremely accurate eigenvalues. Thus the $M A F$ solution gives an accurate description of the wave function as well as of the eigenvalues. 
The monograph contains many examples to illustrate the methods and to allow a comparison between the WKB and the MAF solutions. In addition, we include a few problems for the benefit of those who want additional insight. 


\section{WKB SOLUTIONS TO INITIAL VALUE PROBLEMS}

\subsection{Introduction}

This chapter is devoted to the WKB approximation to the onedimensional wave equation,

$$
\frac{d^{2} \Psi(x)}{d x^{2}}+\Gamma^{2}(x) \psi(x)=0,
$$

where $\Psi$ represents the electromagnetic field or the wave function and $\Gamma^{2}(x)$ is assumed to be "slowly varying" (we will quantify this assumption later). These $W K B$ solutions are not valid near the turning points, where $\Gamma^{2}(x)=0$. We then discuss the formulas that facilitate the connection between the two WKB solutions, one on each side of the turning point. Finally, we will illustrate the use of the connection formulas in solving initial value problems. In Chapters 4 and 6 we will show how the use of modified Airy functions leads to an improved solution (in most cases) that precludes the need for connection formulas.

\subsection{The WKB Solutions}

If a plane electromagnetic wave propagating in the $x$ direction is incident normally onto an inhomogeneous medium having refractive index $n(x)$, the electric field satisfies the equation

$$
\frac{d^{2} \psi(x)}{d x^{2}}+\frac{\omega^{2}}{c^{2}} n^{2}(x) \psi(x)=0,
$$


where $\Psi(x)$ is either $E_{y}$ or $E_{z}$; the $E_{x}$ component is taken to be 0 . Thus, $\Gamma^{2}(x)$ of Eq. (1) is

$$
\Gamma^{2}(x)=\frac{\omega^{2}}{c^{2}} n^{2}(x)=\frac{4 \pi^{2} n^{2}(x)}{\lambda_{0}^{2}},
$$

where

$$
\lambda_{0}=\frac{2 \pi c}{\omega}
$$

represents the free-space wavelength. If $\Gamma^{2}(x)$ is a constant (that is, if the medium is homogeneous), the solution (aside from a multiplying constant) is given by

$$
\Psi(x)=\exp ( \pm i \Gamma x) \text {. }
$$

This suggests that, for the nonuniform medium $\left[\Gamma^{2}=\Gamma^{2}(x)\right]$, we try a solution of the form

$$
\psi(x)=\exp [ \pm i u(x)]
$$

where $u(x)$ is to be determined. Substituting Eq. (6) into Eq. (1) yields

$$
i u^{\prime \prime}-\left(u^{\prime}\right)^{2}+\Gamma^{2}(x)=0,
$$

where primes denote differentiation with respect to $x$. Equation (7) is exact. If $\Gamma^{2}$ is a constant, then $u^{\prime \prime}=0$; thus, when $\Gamma^{2}(x)$ is slowly varying, we may assume $u^{\prime \prime}$ to be negligible in comparison to $\left(u^{\prime}\right)^{2}$ to obtain

$$
\left(u^{\prime}\right)^{2}=\Gamma^{2}(x) .
$$

The solution of this equation is 


$$
u(x)= \pm \int^{x} \Gamma(x) d x
$$

Thus, in this approximation, we obtain

$$
\Psi(x) \approx \Psi_{0}(x)=\exp \left[ \pm i \int^{x} \Gamma(x) d x\right],
$$

which is known as the zeroth-order WKB approximation. The physical interpretation of Eq. (10) is obvious: the inhomogeneous region is divided into a series of homogeneous slabs, each of which has thickness $\Delta x$ in which $\Gamma=\Gamma(x)$. The total phase change is then the summation of the incremental phase changes which, in the limit $\Delta x \rightarrow 0$, becomes the integral on the right side of Eq. (10).

To proceed, we use the differential equation satisfied by $\Psi_{0}(\mathrm{x})$; differentiation gives

$$
\frac{d^{2} \Psi_{0}(x)}{d x^{2}}+\left[\Gamma^{2}(x) \mp i \frac{d \Gamma}{d x}\right] \Psi_{0}(x)=0 .
$$

Equation (11) is the same as Eq. (1), provided that we assume

$$
\left|\frac{d \Gamma}{d x}\right| \ll \Gamma^{2}(x) \text {, }
$$

or

$$
\left|\frac{1}{\Gamma} \frac{d \Gamma}{d x}\right| \ll \Gamma(x) .
$$

This is the condition for the validity of the WKB solution. It quantifies the "slowly varying" condition referred to in the opening paragraph of section 2.1. If we use the relation 


$$
\Gamma(x)=\frac{2 \pi}{\lambda_{0}} n(x),
$$

Eq. (12) becomes

$$
\left|\frac{1}{n} \frac{d n}{d x}\right| \ll \frac{1}{\lambda_{0}}
$$

or

$$
\left|\frac{d n}{d x} \lambda\right| \ll n,
$$

where $\lambda=\lambda_{0} / n$ is the wavelength in the medium. Equation (16) implies that the change in the refractive index in a distance of a wavelength should be very small compared to the refractive index. This is another condition for the validity of the WKB approximation or the "slowly varying" condition.

To obtain the more accurate higher-order solution, we modify the original assumption [Eq. (10)] and write

$$
\psi(x)=F(x) \psi_{0}(x)=F(x) \exp \left[ \pm i \int^{x} \Gamma(x) d x\right],
$$

where, in the spirit of the WKB approximation, $F(x)$ must be slowly varying. To determine $F(x)$, we substitute Eq. (17) into Eq. (1) and neglect the term involving $d^{2} F / d x^{2}$ to obtain

$$
\frac{1}{F} \frac{d F}{d x}=\frac{-1}{2 \Gamma(x)} \frac{d \Gamma(x)}{d x},
$$

or

$$
\frac{d}{d x}[\ln (F \sqrt{\Gamma(x)})]=0 .
$$

Thus, 


$$
F(x)=\frac{\text { const }}{\sqrt{\Gamma(x)}} .
$$

The first-order $W K B$ solutions thus become

$$
\Psi(x)=\frac{1}{\sqrt{\Gamma(x)}} \exp \left[ \pm i \int^{x} \Gamma(x) d x\right] .
$$

Equation (21) is the equation normally meant when reference is made to the WKB solutions. Differentiating twice, we obtain

$$
\frac{d^{2} \psi(x)}{d x^{2}}+\left[\Gamma^{2}(x)+\frac{1}{2 \Gamma} \frac{d^{2} \Gamma}{d x^{2}}-\frac{3}{4 \Gamma^{2}}\left(\frac{d \Gamma}{d x}\right)^{2}\right] \psi(x)=0
$$

Equation (22) is the same as Eq. (1), provided that

$$
\left|\frac{1}{2 \Gamma} \frac{d^{2} \Gamma}{d x^{2}}-\frac{3}{4 \Gamma^{2}}\left(\frac{d \Gamma}{d x}\right)^{2}\right|<<\Gamma^{2}(x) \text {. }
$$

Clearly the first and second derivatives of $\Gamma(x)$ must both be much smaller than $\Gamma^{2}(x)$ in this approximation. 


\subsection{An Alternative Derivation}

We now give an alternative (and a more rigorous) derivation of the $W K B$ solution. For this purpose we assume a scenario according to Eq. (2). Thus, we consider the equation:

$$
\frac{d^{2} \psi}{d x^{2}}+\frac{n^{2}(x)}{t_{0}^{2}} \psi(x)=0,
$$

where

$$
\hbar_{0} \equiv \frac{\lambda_{0}}{2 \pi} \quad \text { and } \quad \Gamma(x)=\frac{n(x)}{\hbar_{0}} .
$$

We try a solution of the form

$$
\psi(x)=\exp \left[\frac{i S(x)}{\hbar_{0}}\right],
$$

where $S(x)$ is to be determined. Substituting Eq. (26) into Eq. (24), we obtain

$$
-\left(\frac{S^{\prime}}{\hbar_{0}}\right)^{2}+i \frac{S^{\prime \prime}}{\hbar_{0}}+\frac{n^{2}(x)}{\hbar_{0}^{2}}=0 .
$$

We next expand $S(x)$ in a power series in $\lambda_{0}$ :

$$
S(x)=S_{0}(x)+\lambda_{0} S_{1}(x)+\hbar_{0}^{2} S_{2}(x)+\cdots .
$$

The logic of this expansion is as follows: If $\pi_{0} \rightarrow 0$, then virtually any refractive index variation is slowly varying and the WKB approximation is accurate. Indeed, in the limit of $\boldsymbol{x}_{0} \rightarrow 0$, we have the geometrical optics approximation. Substituting Eq. (28) into Eq. (27), we obtain 


$$
\begin{aligned}
& {\left[S_{0}^{\prime}(x)+\varkappa_{0} S_{1}^{\prime}(x)+\star_{0}^{2} S_{2}^{\prime}(x)+\cdots\right]^{2}} \\
& -i \varkappa_{0}\left[S_{0}^{\prime \prime}+\varkappa_{0} S_{1}^{\prime \prime}+\chi_{0}^{2} S_{2}^{\prime \prime}+\cdots\right]-n^{2}(x)=0 .
\end{aligned}
$$

Since Eq. (29) must be satisfied for all values of $\lambda_{0}$, the coefficient of each power of $\varkappa_{0}$, must be 0 . This leads to

$$
\begin{gathered}
\left(\frac{d S_{0}}{d x}\right)^{2}=n^{2}(x), \\
2 \frac{d S_{0}}{d x} \frac{d S_{1}}{d x}-i \frac{d^{2} S_{0}}{d x^{2}}=0, \\
\left(\frac{d S_{1}}{d x}\right)^{2}+2 \frac{d S_{0}}{d x} \frac{d S_{2}}{d x}-i \frac{d^{2} S_{1}}{d x^{2}}=0,
\end{gathered}
$$

and so forth. The solution of Eq. (30) is:

$$
\frac{d S_{0}}{d x}= \pm n(x),
$$

SO

$$
S_{0}(x)= \pm \int^{x} n(x) d x \text {. }
$$

From Eq. (31) we obtain

$$
\begin{aligned}
\frac{d S_{1}}{d x} & =\frac{i}{2} \frac{1}{\left(d S_{0} / d x\right)} \frac{d}{d x}\left(\frac{d S_{0}}{d x}\right) \\
& =\frac{i}{2 n} \frac{d n}{d x},
\end{aligned}
$$




$$
S_{1}(x)=\frac{i}{2} \ln [n(x)]+\text { constant }
$$

Therefore,

$$
\exp \left[i S_{1}(x)\right]=\frac{\text { const }}{\sqrt{n(x)}}
$$

Retaining terms up to $S_{1}$, we obtain for $\Psi$

$$
\begin{aligned}
\Psi(x) & =\exp \left[\frac{i S_{0}}{\lambda_{0}}+i S_{1}\right] \\
& =\frac{\text { const }}{\sqrt{n(x)}} \exp \left[ \pm \frac{i}{\lambda_{0}} \int^{x} n(x) d x\right], \\
& =\frac{\text { const }}{\sqrt{\Gamma(x)}} \exp \left[ \pm i \int^{x} \Gamma(x) d x\right]
\end{aligned}
$$

where we have used Eq. (25). These again represent the WKB solutions [cf. Eq. (21)]. Using Eq. (35) and its differential in Eq. (32), we readily obtain

$$
S_{2}(x)= \pm \int^{x}\left[\frac{3}{8 n^{3}}\left(n^{\prime}\right)^{2}-\frac{1}{4} n^{2} n^{\prime \prime}\right] d x
$$

Similarly, 


$$
S_{3}(x)=i\left[-\frac{3}{16} \frac{\left(n^{\prime}\right)^{2}}{n^{4}}+\frac{1}{8 n^{3}} n^{\prime \prime}\right] .
$$

These equations give further insight into the concept of "slowly varying", since they are the terms that are neglected in the WKB solution.

\subsection{The General WKB Solutions}

The general $W K B$ solution is the linear combination

$$
\begin{gathered}
\Psi(x)=\frac{C_{1}}{\sqrt{\Gamma(x)}} \exp \left[i \int^{x} \Gamma(x) d x\right]+ \\
\frac{C_{2}}{\sqrt{\Gamma(x)}} \exp \left[-i \int^{x} \Gamma(x) d x\right] .
\end{gathered}
$$

We could also have used the sine and cosine solutions:

$$
\begin{gathered}
\Psi(x)=\frac{C_{1}}{\sqrt{\Gamma(x)}} \sin \left(\int^{x} \Gamma d x\right)+ \\
\frac{C_{2}}{\sqrt{\Gamma(x)}} \cos \left(\int^{x} \Gamma d x\right) .
\end{gathered}
$$

We have, up to now, assumed that $\Gamma^{2}(x)$ is positive. For $\Gamma^{2}(x)<0$, we write Eq. (1) as

$$
\frac{d^{2} \psi}{d x^{2}}-k^{2}(x) \psi(x)=0,
$$

where 


$$
k^{2}(x)=-\Gamma^{2}(x)
$$

Proceeding in a manner similar to that used to obtain Eq. (21), we obtain the following WKB solution of Eq. (43):

$$
\psi(x)=\frac{D_{1}}{\sqrt{\kappa(x)}} \exp \left[\int^{x} \kappa(x) d x\right]+\frac{D_{2}}{\sqrt{\kappa(x)}} \exp \left[-\int^{x} \kappa(x) d x\right]
$$

This solution is valid when an equation similar to Eq. (12) is satisfied for $\mathbf{k}(x)$. Obviously, they will not be valid near a turning point, where $\kappa^{2}$ or $\Gamma^{2}(x)=0$. To be specific, we consider a variation of $\Gamma^{2}(x)$ of the form shown in Fig. 2.1.

The point $x=a$ represents the turning point. Near $x=a$, the WKB solutions are not valid. For $x<<a$, the solutions are given by Eqs. (41) and (42) and by Eq. (45) for $x>>a$. These solutions on one side of the turning point must now be matched to the solutions on the other side. The matching is done through the connection formulas given below; these formulas are justified in the next chapter.

\section{Case I: Barrier to the Right}

For reasons that will become clear later, the variation of $\Gamma^{2}(x)$ given in Fig. 2.1 is often called "barrier to the right." The connection formulas are: 


$$
\begin{gathered}
\frac{2}{\sqrt{\Gamma(x)}} \sin \left[\int_{x}^{a} \Gamma(x) d x+\frac{\pi}{4}\right] \longleftrightarrow \longrightarrow \frac{1}{\sqrt{\kappa(x)}} \\
\exp \left[-\int_{a}^{x} \kappa(x) d x\right], \\
\frac{1}{\sqrt{\Gamma(x)}} \cos \left[\int_{x}^{a} \Gamma(x) d x+\frac{\pi}{4}\right] \longleftrightarrow \longrightarrow \frac{1}{\sqrt{\kappa(x)}} \\
\exp \left[+\int_{a}^{x} \kappa(x) d x\right] .
\end{gathered}
$$

Thus, the WKB solution given by

$$
\frac{2}{\sqrt{\Gamma(x)}} \sin \left[\int_{a}^{x} \Gamma d x+\frac{\pi}{4}\right]
$$

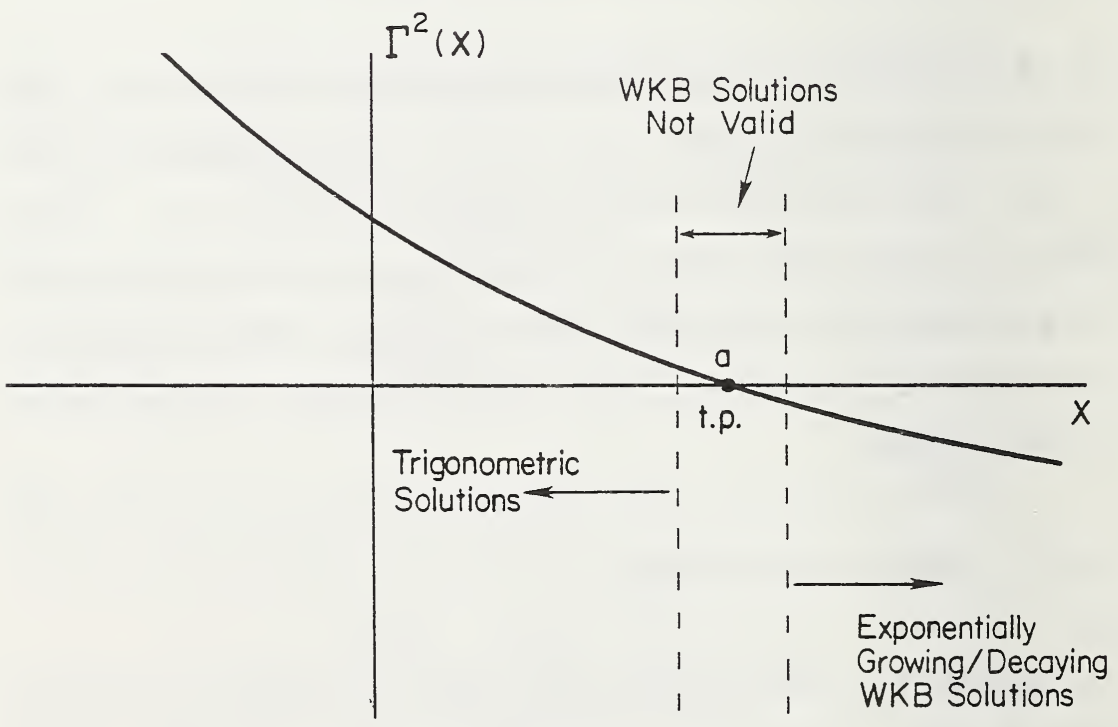

Figure 2.1 $\Gamma^{2}(x)$ variation corresponding to barrier to the right. 
becomes an exponentially decaying solution and the cosine solution becomes the exponentially growing solution. The connection formulas are safe to use only in the direction of the double arrow. If they are used in the direction of the single arrow, an error may be introduced. This can be understood by introducing a small phase term in the argument of the sine function.

$$
\begin{aligned}
& \frac{2}{\sqrt{\Gamma(x)}} \sin \left[\int_{x}^{a} \Gamma(x) d x+\frac{\pi}{4}+\epsilon\right] \\
& =\frac{2}{\sqrt{\Gamma(x)}} \sin \left[\int_{x}^{a} \Gamma(x) d x+\frac{\pi}{4}\right] \cos \epsilon \\
& +\frac{2}{\sqrt{\Gamma(x)}} \cos \left[\int_{x}^{a} \Gamma(x) d x+\frac{\pi}{4}\right] \sin \epsilon \\
& \longrightarrow \frac{\cos \epsilon}{\sqrt{\kappa(x)}} \exp \left[-\int_{a}^{x} k(x) d x\right] \\
& +\frac{2 \sin \epsilon}{\sqrt{K(x)}} \exp \left[+\int_{a}^{x} k(x) d x\right]
\end{aligned}
$$

Eventually the exponentially growing term overwhelms the exponentially decaying term so we may neglect the latter; we thus obtain 


$$
\begin{gathered}
\frac{2}{\sqrt{\Gamma(x)}} \sin \left[\int_{x}^{a} \Gamma(x) d x+\frac{\pi}{4}+\epsilon\right] \longrightarrow \\
\frac{2 \sin \epsilon}{\sqrt{x(x)}} \exp \left[\int_{a}^{x} \kappa(\ddot{x}) d x\right] .
\end{gathered}
$$

Hence, a negligible phase change in the wave function that is valid for $x<<a$ [left side of Eq. (46)] leads to an exponentailly increasing wave function for $x>>a$ (instead of an exponentially decreasing function) if the connection formula is used in the direction of the single arrow. Similarly, we add a term

$$
\frac{1}{\sqrt{\kappa(x)}} \exp \left[-\int_{a}^{x} \kappa(x) d x\right]
$$

on the right side of Eq. (47). With this term, which is negligible for $x>>a$, and using Eq. (46), we obtain

$$
\begin{gathered}
\frac{1}{\sqrt{\Gamma}} \cos \left(\int_{x}^{a} \Gamma d x+\frac{\pi}{4}\right) \\
+\frac{2}{\sqrt{\Gamma}} \sin \left(\int_{x}^{a} \Gamma d x+\frac{\pi}{4}\right)<-\frac{1}{\sqrt{\kappa}} \exp \left[+\int_{a}^{x} \kappa d x\right] \\
+\frac{1}{\sqrt{\kappa}} \exp \left[-\int_{a}^{x} \kappa d x\right]
\end{gathered}
$$




$$
\begin{gathered}
\sqrt{\frac{5}{\Gamma}} \sin \left(\int_{x}^{a} \Gamma d x+\frac{\pi}{4}+\alpha\right) \\
<\frac{1}{\sqrt{k}} \exp \left[\int_{a}^{x} \kappa d x\right]+\frac{1}{\sqrt{k}} \exp \left[-\int_{a}^{x} \kappa d x\right],
\end{gathered}
$$

where

$$
\alpha=\sin ^{-1} \frac{1}{\sqrt{5}} \text {. }
$$

Hence, in this case, the addition of a negligible decaying term in the solution for the region $x>>a$ changes the corresponding solution in the region $x<<a$ significantly if we use the connection formula in the direction of the single arrow.

It is for this reason that the connection formulas should generally be used in the direction of the double arrow [Fröman and Fröman (1965) and Pauli (1980)]. Nevertheless, the connection formulas [given by Eqs. (46) and (47)] have been extensively used in the reverse direction also and accurate results have been obtained (see Chapter 5).

\section{Case II: Barrier to the Left}

Consider next the variation of $\Gamma^{2}(x)$ as given by Fig. 2.2 ; this is often called "barrier to the left." The solutions are exponential on the left of $x=a$ and sine or cosine functions on the right of $x=a$. The connection formulas are 


$$
\begin{gathered}
\frac{1}{\sqrt{\kappa(x)}} \exp \left[-\int_{x}^{a} k(x) d x\right] \longleftrightarrow \longrightarrow \\
\frac{2}{\sqrt{\Gamma(x)}} \sin \left[\int_{a}^{x} \Gamma(x) d x+\frac{\pi}{4}\right],
\end{gathered}
$$

Exponentially Growing/Decaying WKB Solutions

WKB Solutions

Not Valid

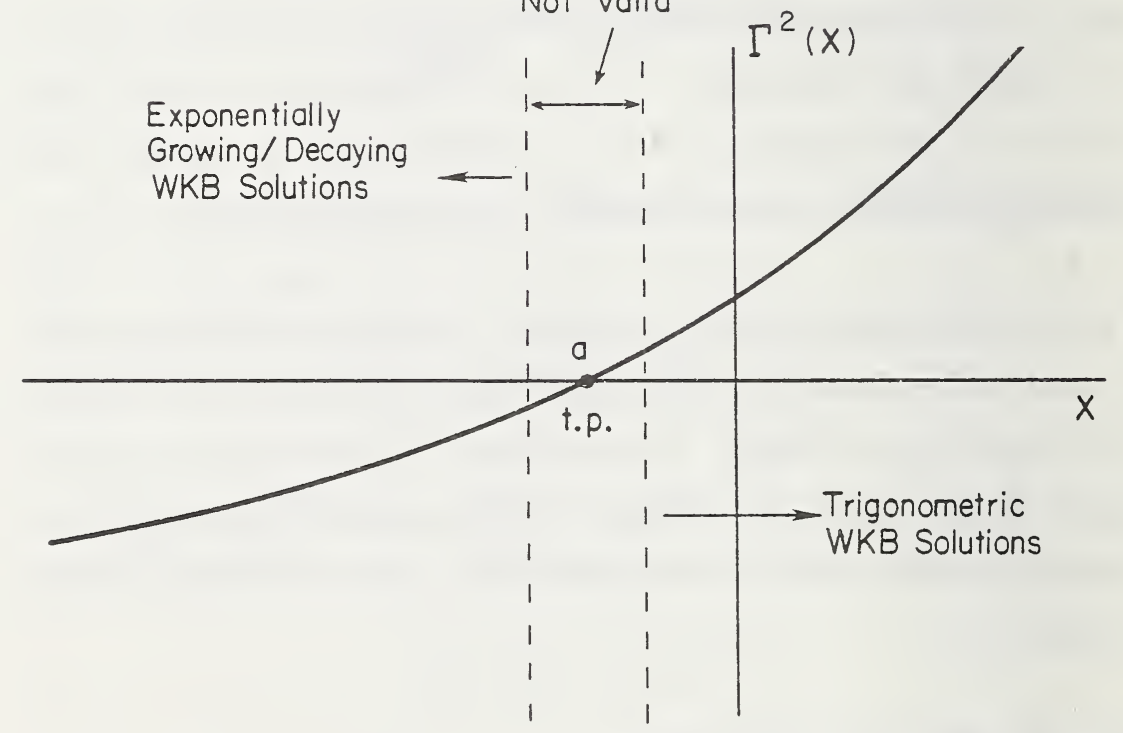

Figure 2.2 $\Gamma^{2}(x)$ variation corresponding to barrier to the left. 


$$
\begin{gathered}
\frac{1}{\sqrt{\kappa(x)}} \exp \left[+\int_{x}^{a} \kappa(x) d x\right] \ll \\
\frac{1}{\sqrt{\Gamma(x)}} \cos \left[\int_{a}^{x} \Gamma(x) d x+\frac{\pi}{4}\right] .
\end{gathered}
$$

These formulas are again safe to use only in the direction of the double arrow; however, we will use them in both directions.

We illustrate the use of the connection formulas through examples.

\subsection{Examples}

\section{Example 2.1}

In this example (see Fig. 2.3)

$$
\Gamma^{2}(x)=2-x,
$$

and the boundary conditions are $\Psi(0)=1$ and $\Psi^{\prime}(0)=0$. Thus, $x=2$ represents the turning point. In the region $x<2$ the solutions are oscillatory and for $x>2$, the solutions are exponentially growing and exponentially decaying. To find the $W K B$ solutions in the region $x<2$, consider the following. Let 


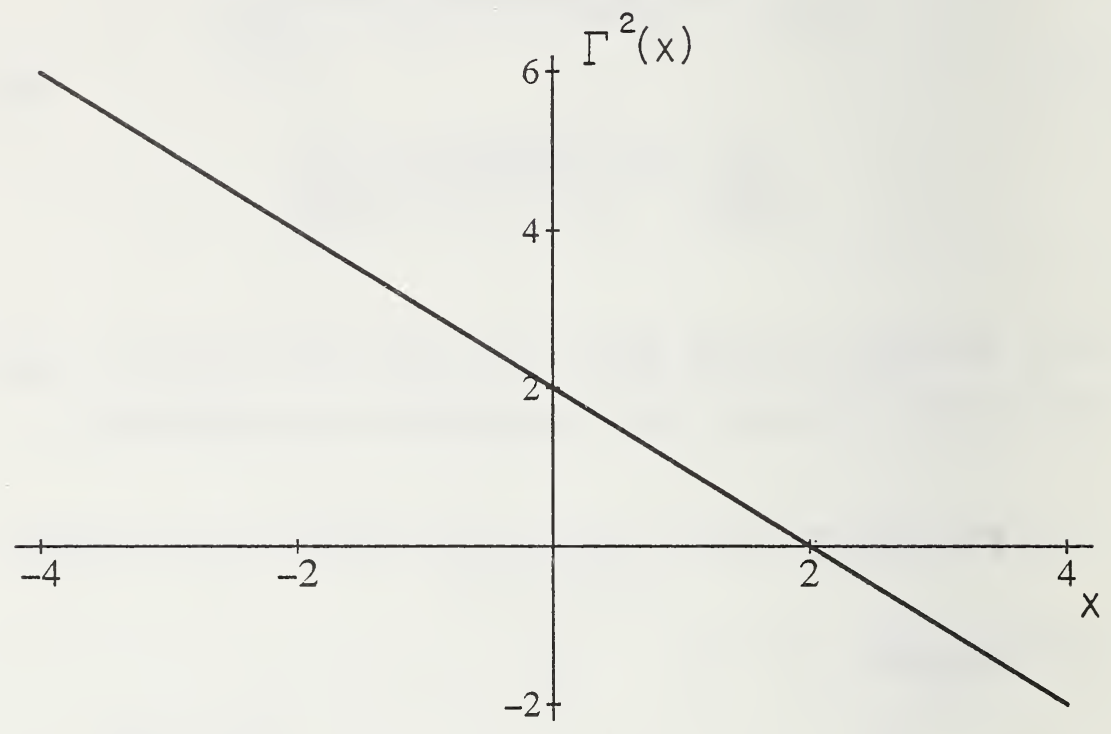

Figure $2.3 \Gamma^{2}(x)=2-x$. See Example 2.1.

$$
\begin{aligned}
\eta(x) & =\int_{x}^{2} \Gamma(x) d x \\
= & \int_{x}^{2}(2-x)^{1 / 2} d x=\frac{2}{3}(2-x)^{3 / 2} .
\end{aligned}
$$

Thus, for $x<2$ 


$$
\begin{aligned}
\Psi_{W K B} & =\frac{A}{\sqrt{\Gamma(x)}} \sin \left[\int_{x}^{2} \Gamma(x) d x+\alpha\right] \\
& =\sqrt{\frac{\Gamma(0)}{\Gamma(x)}} \frac{\sin [\eta(x)+\alpha]}{\sin [\eta(0)+\alpha]} \\
=\left(\frac{2}{2-x}\right)^{1 / 4} \frac{\sin \left[\frac{2}{3}(2-x)^{3 / 2}+\alpha\right]}{\sin \gamma} &
\end{aligned}
$$

where

$$
\gamma=\frac{4 \sqrt{2}}{3}+\alpha,
$$

and we have used the condition $\Psi(0)=1$. The other initial condition $\left(\Psi^{\prime}(0)=0\right)$ gives

$$
\alpha=\tan ^{-1}(8 \sqrt{2})-\frac{4}{3} \sqrt{2} .
$$

In the region $x>2$, we define

$$
\begin{gathered}
\mathrm{\kappa}^{2}(x)=-\Gamma^{2}(x)=x-2, \\
\zeta(x)=\int_{2}^{x} \kappa(x) d x=\int_{2}^{x}(x-2)^{1 / 2} d x \\
=\frac{2}{3}(x-2)^{3 / 2} .
\end{gathered}
$$

We now use the connection formulas to obtain the exponentially decaying and growing solutions for $x>2$. To that end, we write the solution given by Eq. (57) in terms of the left side of Eqs. (46) and (47). Thus 


$$
\begin{aligned}
& \frac{1}{\sqrt{\Gamma(x)}} \sin \left[\int_{x}^{2} \Gamma(x) d x+\alpha\right] \\
& =\frac{1}{\sqrt{\Gamma(x)}} \sin \left[\left(\int_{x}^{2} \Gamma(x) d x+\frac{\pi}{4}\right)+\left(\alpha-\frac{\pi}{4}\right)\right] \\
& =\sin \left(\alpha-\frac{\pi}{4}\right) \frac{1}{\sqrt{\Gamma(x)}} \cos \left[\int_{x}^{2} \Gamma(x) d x+\frac{\pi}{4}\right] \\
& +\cos \left(\alpha-\frac{\pi}{4}\right) \frac{1}{\sqrt{\Gamma(x)}} \sin \left[\int_{x}^{2} \Gamma(x) d x+\frac{\pi}{4}\right] \\
& \stackrel{\sin \left(\alpha-\frac{\pi}{4}\right) \frac{1}{\sqrt{\kappa(x)}} \exp \left[\int_{2}^{x} \kappa(x) d x\right]}{+\cos \left(\alpha-\frac{\pi}{4}\right) \frac{1}{2 \sqrt{\kappa(x)}} \exp \left[-\int_{2}^{x} \kappa(x) d x\right] .}
\end{aligned}
$$

The WKB solution for $x>2$ is thus given by

$$
\begin{aligned}
& \Psi_{W K B}(x)=\frac{2^{1 / 4}}{\sin \gamma}\left[\frac{\sin \left(\alpha-\frac{\pi}{4}\right)}{(x-2)^{1 / 4}} \exp [\zeta(x)]\right. \\
& +\frac{\cos \left(\alpha-\frac{\pi}{4}\right)}{2(x-2)^{1 / 4}} \exp [-\zeta(x)] \quad \text { for } x>2 .
\end{aligned}
$$


The exact solution of Eq. (55) is given by

$$
\psi=\pi\left[A i(x-2) B i^{\prime}(-2)-B i(x-2) A i^{\prime}(-2)\right],
$$

with $A i^{\prime}(-2)=0.61825902$ and $B i^{\prime}(-2)=0.27879517$. The exact solution given by Eq. (64) is plotted in Fig. 2.4 together with the WKB solution given by Eqs. (57) and (63). The WKB solution agrees with the exact solution away from the turning point $x=2$.

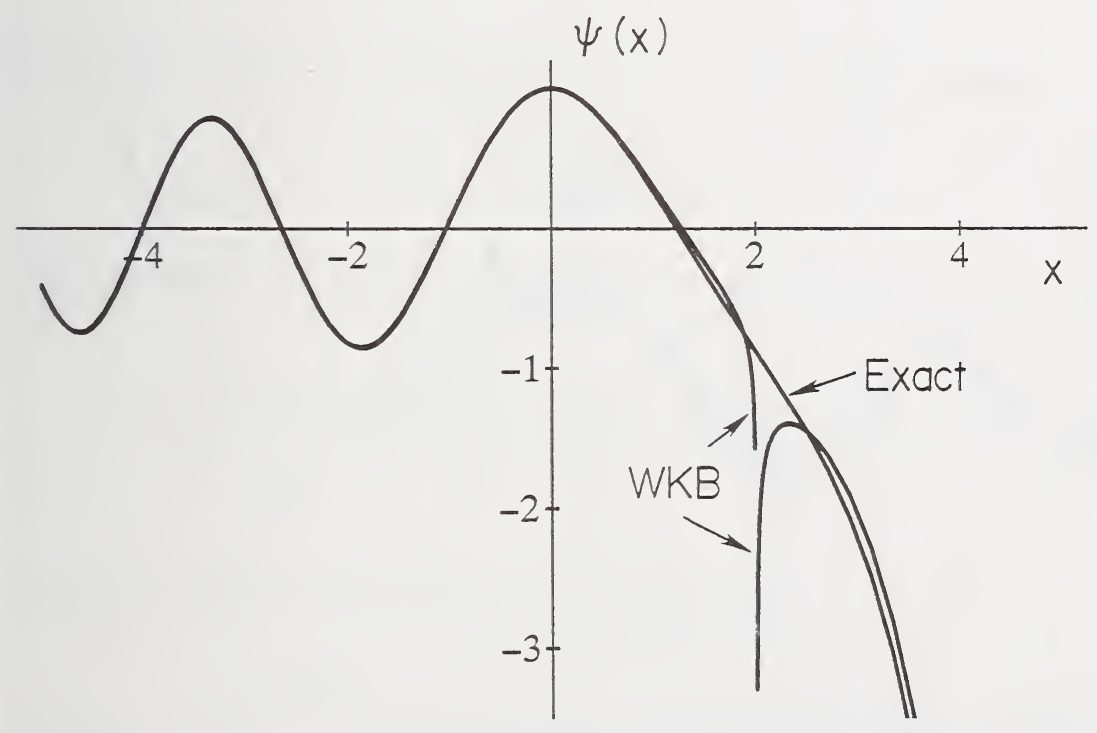

Figure 2.4 Exact and WKB solutions for Example 2.1. 
Care is required in using the WKB (or other approximate) solutions. Consider, for example,

$$
\Gamma^{2}(x)=1-x .
$$

The plots of the WKB and exact solutions (subject to the same initial conditions) are shown in Fig. 2.5. The significant difference in the large $x$ behavior in Fig. 2.5 is because $x=-1$ is very close to the zero of $A i^{\prime}(x)$. Indeed, $A i^{\prime}(-1.0)=-0.01016057, A i^{\prime}(-1.1)=+0.04602915, \quad$ and $A i^{\prime}(-1.0)$ is the coefficient of the growing term $B i(x-1)$ in the exact solution.

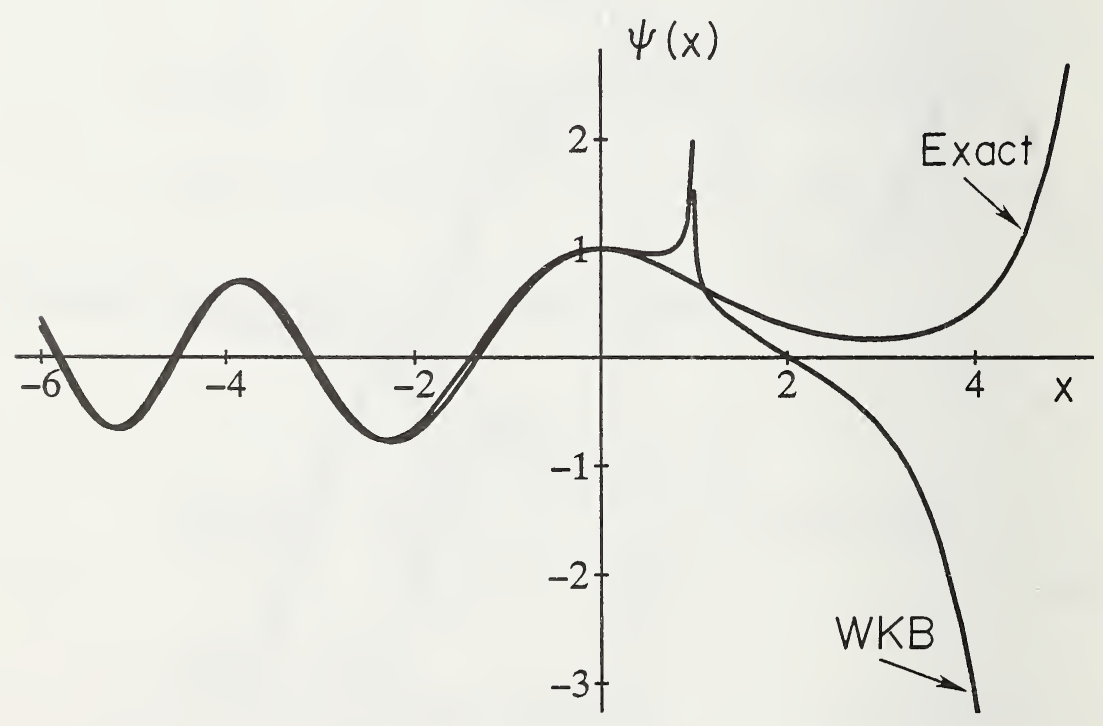

Figure 2.5. Exact and WKB solutions for $\Gamma^{2}(x)=1-x$. Compare with Fig. 2.4. 


\section{Example 2.2}

We next consider the equation

$$
\frac{d 2 \psi}{d x^{2}}+\frac{1}{4}[\exp (-x+a)-1] \psi(x)=0, \quad 0 \leq x<\infty,
$$

subject to the initial conditions

$$
\psi(0)=1 \text { and } \quad \psi^{\prime}(0)=0 .
$$

The exact solutions of Eq. (66) are $J_{1}(\xi)$ and $Y_{1}(\xi)$, where

$$
\xi=\exp \left(\frac{a-x}{2}\right) \text {. }
$$

This can easily be seen by writing the equation for $\Psi$ in terms of $\xi$ :

$$
\xi^{2} \frac{d^{2} \psi}{d \xi^{2}}+\xi \frac{d \psi}{d \psi}+\left(\xi^{2}-1\right) \psi(\xi)=0
$$

Applying the initial conditions, we obtain

$$
\begin{aligned}
\Psi_{\text {EXACT }}(x) & =-\frac{\pi}{4} \beta\left[\left\{Y_{0}(\beta)-Y_{2}(\beta)\right\} J_{1}(\beta \exp (-x / 2))\right. \\
& \left.-\left\{J_{0}(\beta)-J_{2}(\beta)\right\} Y_{1}(\beta \exp (-x / 2))\right]
\end{aligned}
$$

where $\beta=\exp (a / 2)$. Notice that the arguments of $J_{1}$ and $Y_{1}$ are simply $\xi$. To obtain the $W K B$ solutions, we note that $x=a$ is the 
turning point. For $x<a$, the solutions are oscillatory and for $x>a$ they are exponential. Now

$$
\Gamma^{2}(x)=\frac{1}{4}[\exp (-x+a)-1] .
$$

Therefore,

$$
\begin{gathered}
\eta(x)=\int_{x}^{a} \Gamma(x) d x=\frac{1}{2} \int_{x}^{a}[\exp (-x+a)-1]^{1 / 2} d x \\
=\int_{0}^{y} \frac{y^{2} d y}{1+y^{2}}=y-\tan ^{-1} y
\end{gathered}
$$

where

$$
y^{2}=\exp (-x+a)-1=4 \Gamma^{2}(x) .
$$

Therefore,

$$
\eta(x)=\int_{x}^{a} \Gamma(x) d x=2 \Gamma(x)-\tan ^{-1}[2 \Gamma(x)] .
$$

In the region $0<x<a$, we write the solution as

$$
\begin{aligned}
\Psi_{W K B}(x) & =\frac{A}{\sqrt{\Gamma(x)}} \sin \left[\int_{x}^{a} \Gamma(x) d x+\alpha\right] \\
= & \sqrt{\frac{\Gamma(0)}{\Gamma(x)}} \frac{\sin [\eta(x)+\alpha]}{\sin [\eta(0)+\alpha]},
\end{aligned}
$$

where we have used the initial condition $\Psi(0)=1$ and

$$
\Gamma(0)=\frac{1}{2}\left(e^{a}-1\right)^{1 / 2} .
$$

Using the condition $\Psi^{\prime}(0)=0$, we readily obtain 


$$
\alpha=\tan ^{-1}\left\{\frac{16[\Gamma(0)]^{3}}{1+4 \Gamma^{2}(0)}\right\}-\eta(0) .
$$

Thus, the solution in domain $0<x<a$ is completely determined. We now use the connection formulas [Eqs. (46) and (47)] to obtain the solution in the region $x>a$. However, to use the connection formulas we must cast the solution given by Eq. (75) in terms of the left side of Eqs. (46) and (47); thus, we write

$$
\begin{aligned}
& \frac{1}{\sqrt{\Gamma}} \sin \left[\int_{x}^{a} \Gamma d x+\alpha\right] \\
& =\frac{1}{\sqrt{\Gamma}} \sin \left[\left(\alpha-\frac{\pi}{4}\right)+\left(\int_{x}^{a} \Gamma d x+\frac{\pi}{4}\right)\right] \\
& =\sin \left(\alpha-\frac{\pi}{4}\right) \frac{1}{\sqrt{\Gamma(x)}} \cos \left(\int_{x}^{a} \Gamma d x+\frac{\pi}{4}\right) \\
& +\cos \left(\alpha-\frac{\pi}{4}\right) \frac{1}{\sqrt{\Gamma(x)}} \sin \left(\int_{x}^{a} \Gamma d x+\frac{\pi}{4}\right) \\
& \longrightarrow \sin \left(\alpha-\frac{\pi}{4}\right) \frac{1}{\sqrt{\kappa(x)}} \exp \left[+\int_{a}^{x} \kappa d x\right] \\
& +\cos \left(\alpha-\frac{\pi}{4}\right) \frac{1}{2 \sqrt{\kappa(x)}} \exp \left[-\int_{a}^{x} \kappa d x\right] .
\end{aligned}
$$


The WKB solution for $x>a$ is therefore given by

$$
\begin{gathered}
\Psi_{W K B}=\left[\frac{\Gamma(0)}{\kappa(x)}\right]^{1 / 2} \frac{1}{\sin [\eta(0)+\alpha]}\left[\frac{1}{2} \exp [-\zeta(x)]\right. \\
\left.\cos \left(\alpha-\frac{\pi}{4}\right)+\exp [\zeta(x)] \sin \left(\alpha-\frac{\pi}{4}\right)\right]
\end{gathered}
$$

where

$$
\zeta(x)=\int_{a}^{x} \kappa(x) d x=\tan ^{-1}\{2 \kappa(x)\}-2 \kappa(x)
$$

and

$$
\kappa^{2}(x)=\frac{1}{4}[1-\exp (-x+a)]
$$

The WKB solution given by Eqs. (75) and (79) is plotted in Fig. 2.6 together with the exact solution, given by Eq. (70). The WKB solution again deviates from the exact solution near the turning point. 


\section{AIRY FUNCTIONS}

\subsection{Introduction}

In this chapter we will discuss Airy functions and their properties. Airy functions are used in the next chapter to develop the $M A F$ (Modified Airy Function) solutions of the one-dimensional wave equation. We will also use the asymptotic forms of the Airy functions to justify the connection formulas used in the previous chapter.

\subsection{The Airy Functions}

The Airy functions $A i(x)$ and $B i(x)$ are the two independent solutions of the differential equation

$$
\frac{d^{2} \psi}{d x^{2}}-x \psi(x)=0
$$

The Airy functions are defined through the following equations:

$$
\begin{gathered}
A i(x)=a_{1} f(x)-a_{2} g(x), \\
B i(x)=\sqrt{3}\left[a_{1} f(x)+a_{2} g(x)\right],
\end{gathered}
$$




$$
\begin{aligned}
f(x)=1 & +\frac{1}{3 !} x^{3}+\frac{1 \cdot 4}{6 !} x^{6}+\frac{1 \cdot 4 \cdot 7}{9 !} x^{9}+\ldots \\
& =\sum_{0}^{\infty} 3^{k}\left(\frac{1}{3}\right)_{k} \frac{x^{3 k}}{(3 k) !} \\
g(x)=x & +\frac{2}{4 !} x^{4}+\frac{2 \cdot 5}{7 !} x^{7}+\frac{2 \cdot 5 \cdot 8}{10 !} x^{10}+\ldots \\
& =\sum_{0}^{\infty} 3^{k}\left(\frac{2}{3}\right)_{k} \frac{x^{3 k+1}}{(3 k+1) !},
\end{aligned}
$$

where

$$
\begin{gathered}
\left(\alpha+\frac{1}{3}\right)_{0}=1, \\
3^{k}\left(\alpha+\frac{1}{3}\right)_{k}=(3 \alpha+1)(3 \alpha+4) \ldots(3 \alpha+3 k-2) \\
(\alpha \text { arbitrary; } k=1,2,3, \ldots), \\
a_{1}=A i(0)=\frac{B i(0)}{\sqrt{3}}=\frac{3^{-2 / 3}}{\Gamma\left(\frac{2}{3}\right)}=0.355028053887817,
\end{gathered}
$$




$$
a_{2}=A i^{\prime}(0)=\frac{B i^{\prime}(0)}{\sqrt{3}}=\frac{3^{-1 / 3}}{\Gamma\left(\frac{1}{3}\right)}=0.258819403792807
$$

These equations and most of the equations that follow have been adapted from the handbook by Abramowitz and Stegun (1970). The Airy functions and their derivatives are related to the Bessel functions through the following:

$$
\begin{aligned}
\zeta & =\frac{2}{3} x^{3 / 2}, \quad x>0, \\
A i(x) & =\frac{1}{3} \sqrt{x}\left[I_{-1 / 3}(\zeta)-I_{1 / 3}(\zeta)\right] \\
& =\pi^{-1} \sqrt{\frac{x}{3}} K_{1 / 3}(\zeta), \\
A i(-x) & =\frac{1}{3} \sqrt{x}\left[J_{1 / 3}(\zeta)+J_{-1 / 3}(\zeta)\right], \\
A i^{\prime}(x) & =-\frac{1}{3} x\left[I_{-2 / 3}(\zeta)-I_{2 / 3}(\zeta)\right] \\
& =-\pi^{-1}\left(\frac{x}{\sqrt{3}}\right) K_{2 / 3}(\zeta), \\
A i^{\prime}(-x) & =-\frac{1}{3} x\left[J_{-2 / 3}(\zeta)-J_{2 / 3}(\zeta)\right], \\
B i(x) & =\sqrt{\frac{x}{3}}\left[I_{-1 / 3}(\zeta)+I_{1 / 3}(\zeta)\right],
\end{aligned}
$$




$$
\begin{aligned}
& B i(-x)=\sqrt{\frac{x}{3}}\left[J_{-1 / 3}(\zeta)-J_{1 / 3}(\zeta)\right], \\
& B i^{\prime}(x)=\frac{x}{\sqrt{3}}\left[I_{-2 / 3}(\zeta)+I_{2 / 3}(\zeta)\right], \\
& B i^{\prime}(-x)=\frac{x}{\sqrt{3}}\left[J_{-2 / 3}(\zeta)+J_{2 / 3}(\zeta)\right] .
\end{aligned}
$$

The asymptotic forms of the Airy functions and their derivatives are follows:

$$
\begin{gathered}
A i(x) \sim \frac{1}{2} \pi^{-1 / 2} x^{-1 / 4} e^{-\zeta} \sum_{0}^{\infty}(-1)^{k} c_{k} \zeta^{-k} \\
A i(-x) \sim \pi^{-1 / 2} x^{-1 / 4}\left[\sin \left(\zeta+\frac{\pi}{4}\right) \sum_{0}^{\infty}(-1)^{k} c_{2 k} \zeta^{-2 k}\right. \\
\left.-\cos \left(\zeta+\frac{\pi}{4}\right) \sum_{0}^{\infty}(-1)^{k} c_{2 k+1} \zeta^{-2 k-1}\right] \\
A i^{\prime}(x) \sim-\frac{1}{2} \pi^{-1 / 2} x^{1 / 4} e^{-\zeta} \sum_{0}^{\infty}(-1)^{k} d_{k} \zeta^{-k} \\
A i^{\prime}(-x) \sim-\pi^{-1 / 2} x^{1 / 4}\left[\cos \left(\zeta+\frac{\pi}{4}\right) \sum_{0}^{\infty}(-1)^{k} d_{2 k} \zeta^{-2 k}\right. \\
\left.+\sin \left(\zeta+\frac{\pi}{4}\right) \sum_{0}^{\infty}(-1)^{k} d_{2 k+1} \zeta^{-2 k-1}\right]
\end{gathered}
$$




$$
\begin{gathered}
B i(x) \sim \pi^{-1 / 2} x^{-1 / 4} e^{\zeta} \sum_{0}^{\infty} c_{k} \zeta^{-k}, \\
B i(-x) \sim \pi^{-1 / 2} x^{-1 / 4}\left[\cos \left(\zeta+\frac{\pi}{4}\right) \sum_{0}^{\infty}(-1)^{k} c_{2 k} \zeta^{-2 k}\right. \\
\left.+\sin \left(\zeta+\frac{\pi}{4}\right) \sum_{0}^{\infty}(-1)^{k} c_{2 k+1} \zeta^{-2 k-1}\right] \\
B i^{\prime}(x) \sim \pi^{-1 / 2} x^{1 / 4} \exp (\zeta) \sum_{0}^{\infty} d_{k} \zeta^{-k} \\
B i^{\prime}(-x) \sim \pi^{-1 / 2} x^{1 / 4}\left[\sin \left(\zeta+\frac{\pi}{4}\right) \sum_{0}^{\infty}(-1)^{k} d_{2 k} \zeta^{-2 k}\right. \\
\left.-\cos \left(\zeta+\frac{\pi}{4}\right) \sum_{0}^{\Sigma, \infty}(-1)^{k} d_{2 k+1} \zeta^{-2 k-1}\right]
\end{gathered}
$$

where 


$$
\begin{gathered}
c_{0}=1, \\
c_{k}=\frac{\Gamma\left(3 k+\frac{1}{2}\right)}{54^{k} k ! \Gamma\left(k+\frac{1}{2}\right)}=\frac{(2 k+1)(2 k+3) \ldots(6 k-1)}{216^{k} k !} \\
d_{0}=1, \\
d_{k}=-\frac{6 k+1}{6 k-1} c_{k} \quad(k=1,2,3, \ldots) .
\end{gathered}
$$

For numerical evaluation in the following chapters we used the following procedure: (i) for $|x| \leq 1$, we used Eqs. (2) and (3) with the first 10 terms of Eqs. (4) and (5); (ii) for $1<|x| \leq 4$ we used the first 50 terms of Eqs. (4) and (5); (iii) for $|x|>4$, we used the first 10 terms of Eqs. (16) through (23). This gave an accuracy of 1 in $10^{9}$.

\subsection{Asymptotic Forms and the Connection Formulas}

Figures 3.1 and 3.2 show that both $A i(x)$ and $B i(x)$ have damped oscillatory behavior for $x<0$; for $x \rightarrow \infty$ the function $\operatorname{Ai}(x)$ approaches 0 exponentially and the function $B i(x)$ increases exponentially. Consider more closely the first term of the asymptotic series given in Eqs. (16) and (17): 


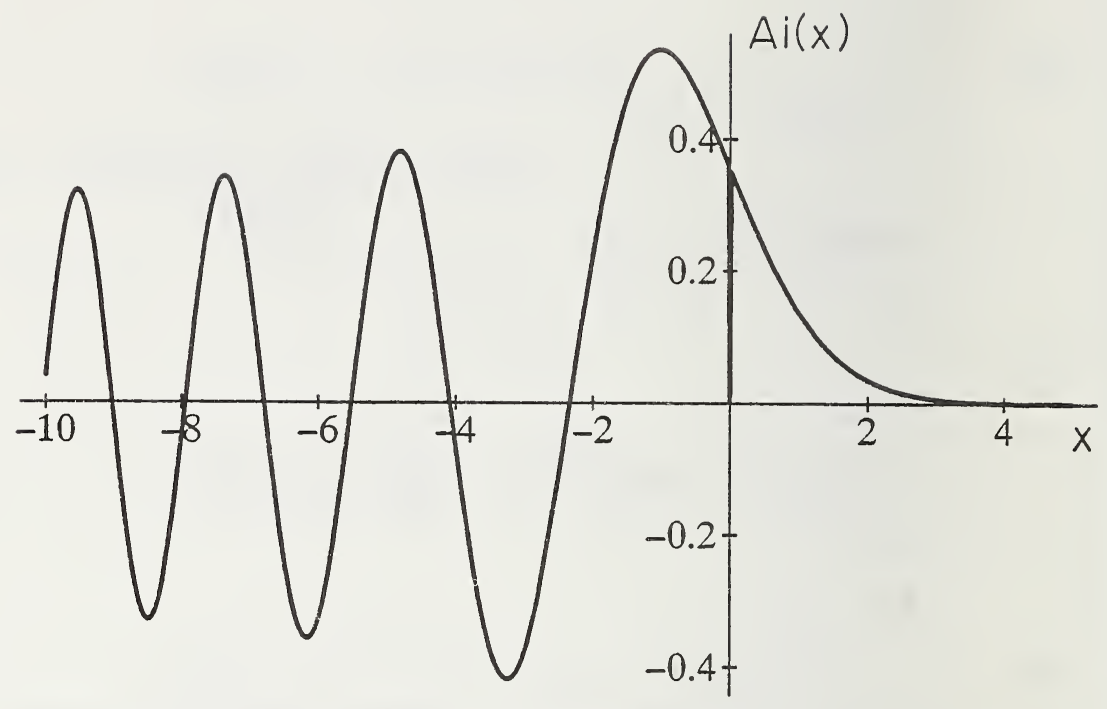

Figure 3.1 Airy function $\operatorname{Ai}(x)$.

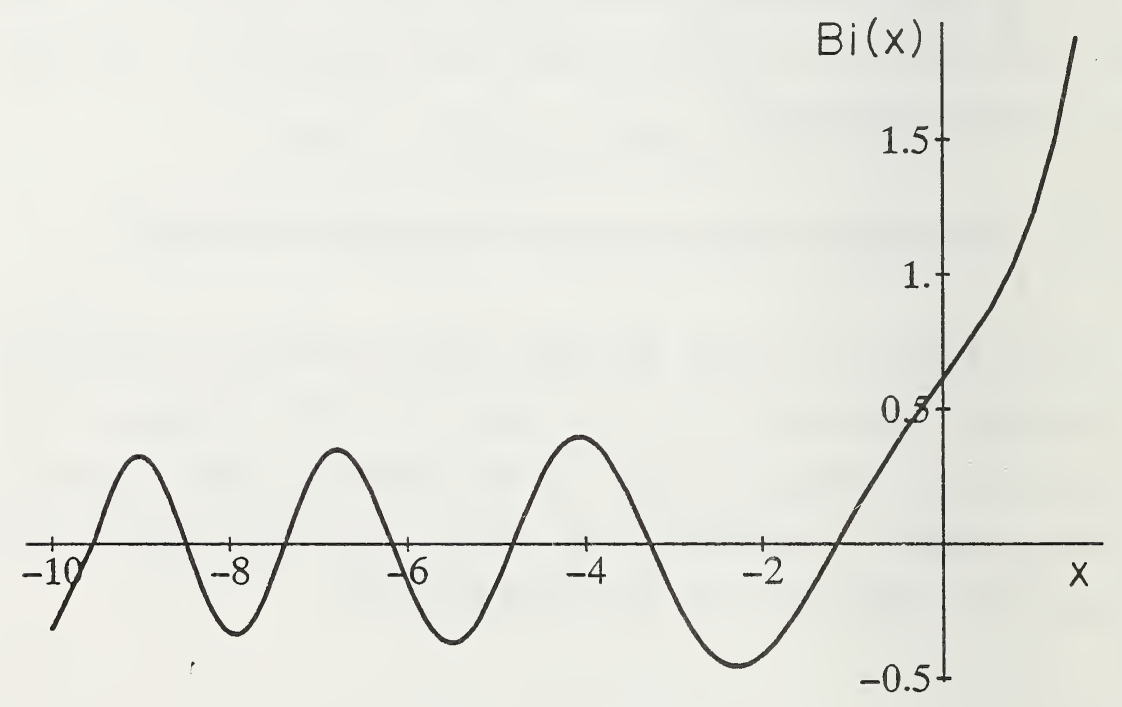

Figure 3.2 Airy function $B i(x)$. 


$$
\begin{gathered}
\text { Ai(x) } \underset{x \rightarrow-\infty}{\longrightarrow} \frac{1}{\pi^{1 / 2}|x|^{1 / 4}} \sin \left[\zeta+\frac{\pi}{4}\right] \\
\underset{x \rightarrow+\infty}{\longrightarrow} \frac{1}{2 \pi^{1 / 2}|x|^{1 / 4}} \exp (-\zeta),
\end{gathered}
$$

where

$$
\zeta=\frac{2}{3}|x|^{3 / 2}
$$

From Eq. (25), we note that the asymptotic forms of $\operatorname{Ai}(x)$ obey the connection formula

$$
\frac{1}{|x|^{1 / 4}} \sin \left[\zeta+\frac{\pi}{4}\right] \longleftrightarrow \longrightarrow \frac{1}{2|x|^{1 / 4}} \exp (-\zeta) \text {. }
$$

Similarly, examination of the asymptotic forms of $B i(x)$, given in Eqs. (20) and (21) leads to

$$
\frac{1}{|x|^{1 / 4}} \cos \left[\zeta+\frac{\pi}{4}\right] \longleftrightarrow \longrightarrow \frac{1}{|x|^{1 / 4}} \exp (+\zeta) \text {. }
$$

Consider next the $W K B$ solutions of Eq. (1). Here,

$$
\Gamma^{2}(x)=-x
$$


Thus, for $x<0$,

$$
\begin{gathered}
\int_{x}^{0} \Gamma(x) d x=\frac{2}{3}|x|^{3 / 2}=\zeta, \\
|\Gamma(x)|^{1 / 2}=|x|^{1 / 4},
\end{gathered}
$$

and, therefore, the WKB solutions are [see Eq. (42) of Chapter 2]

$$
\frac{1}{|x|^{1 / 4}} \sin \zeta \text { and } \frac{1}{|x|^{1 / 4}} \cos \zeta
$$

or any linear combination of them ( $x=0$ represents the turning point). Similarly, for $x>0$,

$$
\kappa^{2}(x)=x,
$$

implying

$$
\int_{0}^{x} \kappa(x) d x=\frac{2}{3} x^{2 / 3}
$$

and

$$
|\kappa(x)|^{-1 / 2}=|x|^{-1 / 4} .
$$

Therefore, the WKB solutions of Eq. (1) are

$$
\frac{1}{|x|^{1 / 4}} \exp (\zeta) \quad \text { and } \quad \frac{1}{|x|^{1 / 4}} \exp (-\zeta) \text {. }
$$

The asymptotic forms of the Airy functions are indeed the WKB solutions and we may write Eqs. (26) and (27) as 


$$
\begin{gathered}
\frac{2}{\sqrt{\Gamma(x)}} \sin \left[\int_{x}^{0} \Gamma(x) d x+\frac{\pi}{4}\right] \ll \longrightarrow \frac{1}{\sqrt{\kappa(x)}} \\
\cdot \exp \left[-\int_{0}^{x} \kappa(x) d x\right]
\end{gathered}
$$

and

$$
\begin{gathered}
\frac{1}{\sqrt{\Gamma(x)}} \cos \left[\int_{x}^{0} \Gamma(x) d x+\frac{\pi}{4}\right] \longleftrightarrow \longrightarrow \frac{1}{\sqrt{\kappa(x)}} \\
\cdot \exp \left[+\int_{0}^{x} \kappa(x) d x\right] .
\end{gathered}
$$

These equations justify the connection formulas given by Eqs. (46) and (47) of Chapter 2. (The significance of the double arrow was discussed in the previous chapter.) Similarly, by assuming $\Gamma^{2}(x)$ $=x$, we can justify the connection formulas given by Eqs. (53) and (54) of Chapter 2. 


\section{MAF SOLUTION TO INITIAL VALUE PROBLEMS}

\subsection{Introduction}

The use of the WKB method in quantum mechanical and optical waveguide problems is well established. The method is useful, in spite of its limitations, because it is inherently simple. Improvements have been offered to the basic method in the past few years, the most notable being the work of Langer (1931). He showed that improvements come about because the Airy function is the exact solution to the equation of interest when $\Gamma^{2}$ is linear in $x$. This is significant inasmuch as it prompts us to seek a solution that becomes exact (that is, reduces to the Airy function) when $\Gamma^{2}$ is a linear function of $x$. This would be in direct correspondence with the WKB methodology, for which the solution becomes exact (that is, reduces to the exponential or trigonometric functions) when $\Gamma^{2}(x)$ is constant.

In this chapter we will lay the groundwork for a method of solving the one-dimensional scalar wave equation based on the work of Langer. The solution we will discuss is the product of a function of $x$ and the Airy function, the argument of which is also a function of $x$.

The MAF solution is quite powerful for problems of practical interest. It does not suffer the disadvantages that the WKB solution suffers at the turning point. We will see from the examples in this and the following chapters that this solution gives a very good 
prediction of the eigenfunction as well as the eigenvalue. With regard to the latter, we will find that the method does not work as well as the WKB method in finding the eigenvalues for the lowerorder modes when the wave equation involves a parabolic index profile (or parabolic potential function in the case of quantum well problems.) This will be seen in the later chapters. For most other profiles, the MAF method works much better than the WKB method. The $W K B$ method, although approximate, is special in this regard since it yields the exact eigenvalues for the untruncated parabolic profile. In Chapter 7, we will show that use of the firstorder perturbation theory, using the present Modified Airy Function solutions, yields extremely accurate eigenvalues. Thus the $M A F$ solution gives an accurate description of the wave function as well as of the eigenvalues.

\subsection{The MAF Solution}

The equation of interest is

$$
\frac{d^{2} \psi}{d x^{2}}+\Gamma^{2}(x) \psi(x)=0,
$$

where $\Gamma^{2}$ depends on the details of the problem at hand. In this chapter, we will consider Eq. (1) as an initial value problem, and, in this section, we will develop the formulation to be used repeatedly in this and the following chapter.

We assume a solution to Eq. (1) of the form 


$$
\Psi(x)=\left\{\begin{array}{cc}
F(x) & A i[\xi(x)] \\
& \text { or } \\
G(x) & B i[\xi(x)]
\end{array}\right.
$$

where, the Airy functions $A i(x)$ and $B i(x)$, discussed in the preceding chapter, are solutions of the equation

$$
\frac{d^{2} f(x)}{d x^{2}}-x f(x)=0 \text {. }
$$

Substitution of Eq. (2) into Eq. (1) gives

$$
\begin{gathered}
F^{\prime \prime}(x) A i(\xi)+2 F^{\prime}(x) A i^{\prime}(\xi) \xi^{\prime}(x)+F(x) A i^{\prime}(\xi) \xi^{\prime \prime} \\
+A i(\xi) F(x)\left[\xi(x)\left[\xi^{\prime}(x)\right]^{2}+\Gamma^{2}(x)\right]=0,
\end{gathered}
$$

where primes denote differentiation with respect to the argument and we have used the relation $A i^{\prime \prime}(\xi)=\xi A i(\xi)$. Equation (4) is rigorously correct. We choose $\xi(x)$ so that

$$
\xi(x)\left[\xi^{\prime}(x)\right]^{2}+\Gamma^{2}(x)=0,
$$

the solution of which gives

$$
\xi(x)=\left\{\int_{x_{0}}^{x}\left(\frac{3}{2}\right) \sqrt{-\Gamma^{2}(x)} d x\right\}^{2 / 3} .
$$

In fact, the general solution of Eq. (5) leaves arbitrary the lower limit of integration in the expression for $\xi(x)$. We take it to be the turning point $x_{0}$, for tactical reasons; that choice renders the solution [Eq. (2)] exact if $\Gamma^{2}(x)$ is a linear function of $x$. 
If we neglect the term that is proportional to $F^{\prime \prime}$ in Eq. (4) (which is the only approximation we will make), Eq. (4) becomes

$$
\begin{aligned}
& 2 F^{\prime}(x) A i^{\prime}(\xi) \xi^{\prime}(x)+F(x) A i^{\prime}(\xi) \xi^{\prime \prime}=0, \\
& \text { or } \quad \frac{d}{d x}[\ln F(x)]=-\frac{1}{2} \frac{d}{d x}\left[\ln \xi^{\prime}(x)\right],
\end{aligned}
$$

the solution of which is

$$
F(x)=\frac{\text { const. }}{\sqrt{\xi^{\prime}(x)}} .
$$

An identical expression is obtained for $G(x)$. Equations (2), (6), and (8) represent the solution that we will discuss.

Equation (6) is valid when $\Gamma^{2}(x)$ is positive or negative. Indeed, if we assume $\Gamma^{2}$ is positive for $x<x_{0}$ and negative for $x>x_{0}$, then

$$
\begin{gathered}
\xi(x)=-\left(\frac{3}{2} \int_{x}^{x_{0}} \Gamma(x) d x\right)^{2 / 3} \quad \text { for } \quad x<x_{0}, \\
\xi(x)=\left(\frac{3}{2} \int_{x_{0}}^{x} \kappa(x) d x\right)^{2 / 3} \quad \text { for } x>x_{0},
\end{gathered}
$$

where

$$
\kappa(x)=\sqrt{-\Gamma^{2}(x)} \quad \text { for } \quad x>x_{0} .
$$

Interchanging the limits of the integrals in Eqs. (9) and (10) does not change the value of $\xi$. Simple manipulations yield 


$$
\begin{aligned}
& \xi^{\prime}(x)=\left[\frac{2}{3 \int_{x}^{x_{0}} \Gamma(x) d x}\right]^{1 / 3} \Gamma(x) \quad \text { for } \quad x<x_{0}, \\
& \xi^{\prime}(x)=\left[\frac{2}{3 \int_{x_{0}}^{x} \kappa(x) d x}\right]^{1 / 3} \kappa(x) \text { for } x>x_{0},
\end{aligned}
$$

and thus

$$
\frac{1}{\sqrt{\xi^{\prime}(x)}}=\frac{|\xi|^{1 / 4}}{\left|\Gamma^{2}(x)\right|^{1 / 4}}
$$

The error introduced by neglecting the term proportional to $F^{\prime \prime}$ in Eq. (4) is seen by substituting

$$
\Psi(x)=\left(\xi^{\prime}\right)^{-1 / 2} \phi(\xi)
$$

into Eq. (1). This gives

$$
\phi^{\prime \prime}(\xi)-\xi \phi(\xi)+\left[\left(\xi^{\prime}\right)^{-3}\left(\frac{3\left(\xi^{\prime \prime}\right)^{2}}{4 \xi^{\prime}}-\frac{1}{2} \xi^{\prime \prime \prime}\right)\right] \phi(\xi)=0 .
$$

The method is therefore valid if

$$
\left|\left(\xi^{\prime}\right)^{-3}\left(\frac{3\left(\xi^{\prime \prime}\right)^{2}}{4 \xi^{\prime}}-\frac{1}{2} \xi^{\prime \prime \prime}\right)\right| \ll|\xi| \text {. }
$$

$\xi$ is given by an integral [see Eq. (6)], so we expect it to be a slowly varying function of $x$; its higher derivatives are therefore likely to be small. Although the inequality does not hold at the turning point, the resulting error is small. 
The approximate solution that we will discuss, then, is

$$
\Psi(x)=C_{1} \frac{A i[\xi(x)]}{\sqrt{\xi^{\prime}(x)}}+C_{2} \frac{B i[\xi(x)]}{\sqrt{\xi^{\prime}(x)}},
$$

where

$$
\xi(x)=\left\{\int_{x_{0}}^{x}\left(\frac{3}{2}\right) \sqrt{-\Gamma^{2}(x)} d x\right\}^{2 / 3},
$$

and $x_{0}$ is the turning point, where $\Gamma^{2}(x)$ changes sign. $C_{1}$ and $C_{2}$ are constants, to be determined from the initial conditions.

\subsection{Examples}

\section{Example 4.1}

To demonstrate the applicability of Eq. (18), we consider an initial value problem. We will give the exact and the $M A F$ results and compare them graphically with the WKB method. Consider the equation

$$
\frac{d^{2} \psi}{d x^{2}}+\frac{1}{4}[\exp (-x+a)-1] \psi(x)=0, \quad 0 \leq x<\infty,
$$

subject to the conditions

$$
\psi(0)=1 \text { and } \psi^{\prime}(0)=0 .
$$

The exact solution is given by 


$$
\begin{aligned}
& \Psi_{\mathrm{EXACT}}(x)=\frac{\pi}{4} \beta\left\{\left[Y_{0}(\beta)-Y_{2}(\beta)\right] J_{1}\left[\beta \exp \left(-\frac{x}{2}\right)\right]\right. \\
& \left.-\left[J_{0}(\beta)-J_{2}(\beta)\right] Y_{1}\left[\beta \exp \left(-\frac{x}{2}\right)\right]\right\} \text { for } 0 \leq x<\infty:
\end{aligned}
$$

where

$$
\beta=\exp \left(\frac{a}{2}\right)
$$

The corresponding WKB solutions were given by Eqs. (75) and (79) in Chapter 2. They will not be repeated here.

The $M A F$ solution is given by

$$
\Psi_{M A F}(x)=\frac{C_{1}}{\sqrt{\xi^{\prime}(x)}} A i(\xi)+\frac{C_{2}}{\sqrt{\xi^{\prime}(x)}} B i(\xi),
$$

where

$$
\begin{aligned}
C_{1} & =\pi\left[\left[\xi^{\prime}(0)\right]^{1 / 2} B i^{\prime}(\xi(0))\right. \\
& \left.-\frac{\xi^{\prime \prime}(0)}{2\left[\xi^{\prime}(0)\right]^{3 / 2}} B i(\xi(0))\right],
\end{aligned}
$$




$$
\begin{aligned}
C_{2}= & -\pi\left[\left[\xi^{\prime}(0)\right]^{1 / 2} A i^{\prime}[\xi(0)]\right. \\
& \left.-\frac{\xi^{\prime \prime}(0)}{2\left[\xi^{\prime}(0)\right]^{3 / 2}} A i[\xi(0)]\right], \\
\xi(x)= & -\left[\frac{3}{4} \int_{x}^{a}[\exp (-x+a)-1]^{1 / 2} d x\right]^{2 / 3},
\end{aligned}
$$

or

$$
\xi(x)=-\left[\frac{3}{2} \eta(x)\right]^{2 / 3} \quad \text { for } 0 \leq x \leq a,
$$

and

$$
\xi(x)=\left[\frac{3}{2} \zeta(x)\right]^{2 / 3} \quad \text { for } a \leq x \leq \infty,
$$

where $\eta(x)$ and $\zeta(x)$ are defined by Eqs. (72) and (80) of Chapter 2. Figure 4.1a shows the three solutions for $a=6$; the $M A F$ approximation agrees very well with the exact results, whereas the $W K B$ solution deviates very much near and at the turning point. In fact, the very small difference between the exact and the MAF solutions cannot be easily seen in Fig. 4.1a. For that reason, we also show the difference between the two solutions in Fig. 4.1b.

\subsection{Summary}

The $M A F$ method is capable of yielding an accurate solution to initial value problems. The veracity of the method was demonstrated by using an example that has an exact solution. 


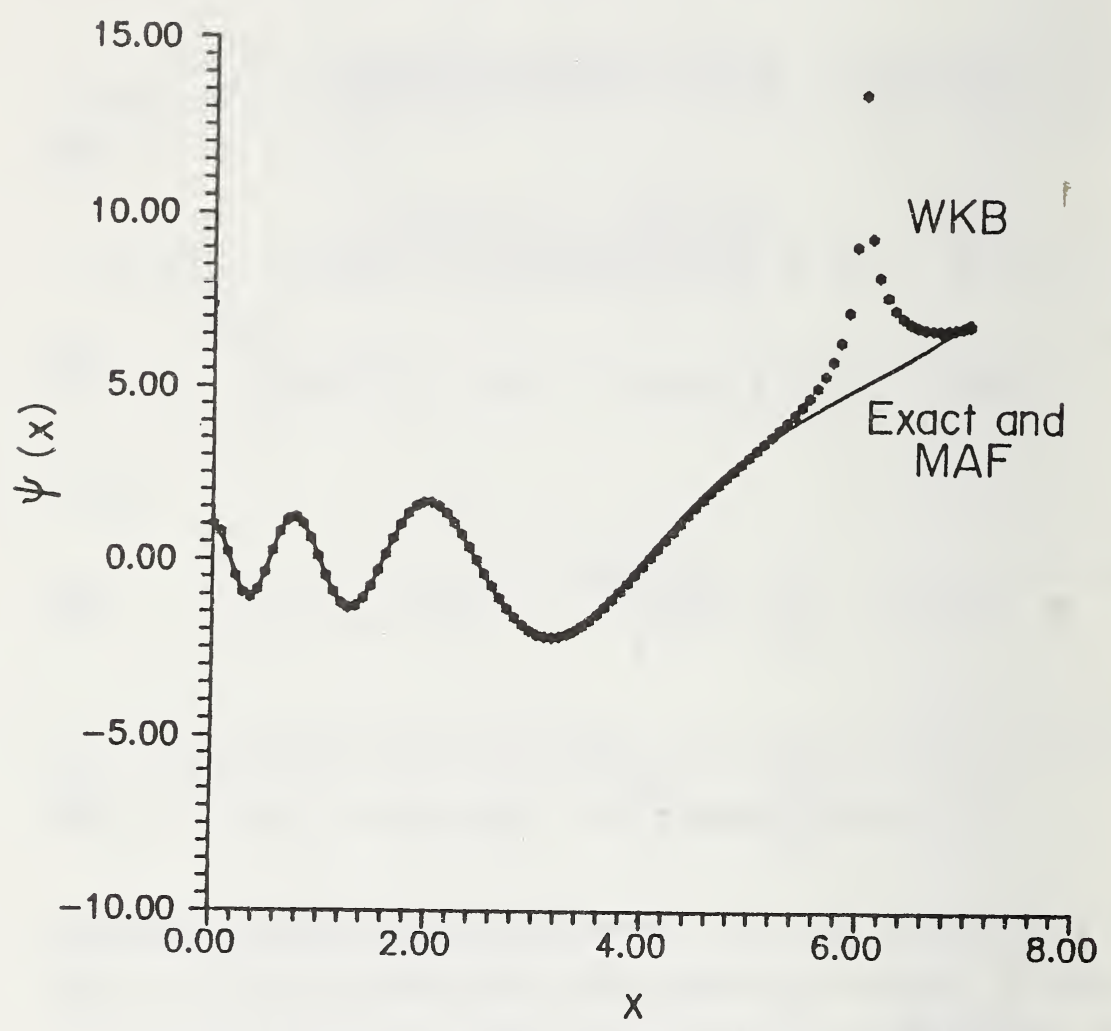

Figure 4.1(a) Exact, $M A F$, and $W K B$ solutions for Example 4.1.

The derivation in this chapter, being based on intuitive concepts, was intended to appeal to the non-mathematically inclined. The original work by Langer (1931), on the other hand, is somewhat pedantic. The salient features of the method, which will be demonstrated further in the following chapters, are these:

1. Equation (18) gives, in a single closed form, an approximation that holds throughout the region of interest, including (but not restricted to) the vicinity of and at the turning points. The first- 


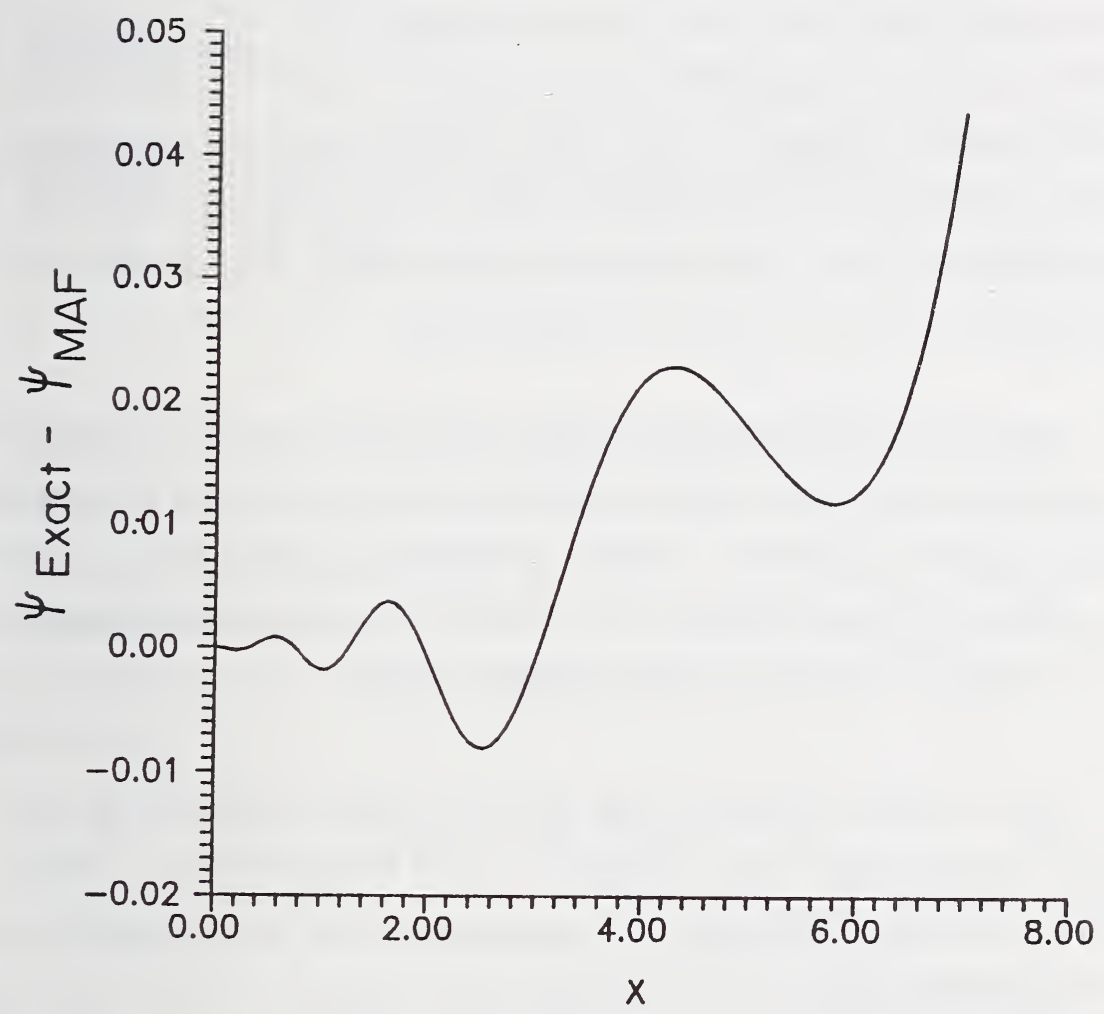

Figure 4.1(b) Difference between the exact and the $M A F$ solutions for Example 4.1.

order $W K B$ solutions fail at and in the vicinity of the turning point. The conventional method of rendering the solution valid everywhere is to approximate $\Gamma^{2}(x)$ by a linear function, $\Gamma^{2}(x)=$ $a+b x$, in the vicinity of the turning points. With this, the approximate solution to the differential equation, aside from constants, is given in terms of Airy functions having argument $-(a+b x) / b^{2 / 3}$. The total solution is expressed in terms of (i) 
oscillatory functions in the regions where $\Gamma^{2}(x)>0$, (ii) exponential functions in the regions where $\Gamma^{2}(x)<0$, and (iii) Airy functions of argument $-(a+b x) / b^{2 / 3}$ in the vicinity of the turning points, where $\Gamma^{2}=0$. The solutions are then connected at the boundaries of the regions. There is invariably a degree of arbitrariness in the selection of the boundary. In contrast, the $M A F$ solution requires no such connection.

2. The solution given here is a single expression in terms of Airy functions, but the argument is not obtained by assuming $\Gamma^{2}(x)$ to be a linear function of $x$. In Eq. (18), the argument of the Airy function is $\xi(x)$ and not $(a+b x) / b^{2 / 3} . \xi(x)$ depends on actual $\Gamma^{2}(x)$ and not on a linear approximation to it.

3. The integration limits of Eq. (6) are chosen to ensure that Eq. (18) reduces to the exact solution if $\Gamma^{2}(x)$ is linear in $x$. Thus, while the usual WKB method becomes exact if $\Gamma^{2}$ is constant, Eq. (18) becomes exact if $\Gamma^{2}(x)$ is linear in $x$.

All of the numerical results of this and the following chapters were obtained on a desktop computer. 


\section{WKB SOLUTIONS TO EIGENVALUE PROBLEMS}

\subsection{Introduction}

For continuity, we again give the equation of interest:

$$
\frac{d^{2} \psi(x)}{d x^{2}}+\Gamma^{2}(x) \psi(x)=0 .
$$

In Chapter 2, we used the WKB method to solve the initial value problem. In this chapter, we consider Eq. (1) to be an eigenvalue problem. The eigenvalue equation appears in many branches of physics and engineering. In quantum mechanics, for example, the one-dimensional Schrödinger equation (see any text on quantum mechanics) is,

$$
\frac{d^{2} \psi}{d x^{2}}+\frac{2 m}{\hbar^{2}}[E-V(x)] \psi(x)=0,
$$

where $\Psi$ represents the wave function describing the particle (of mass $m$ ), $E$ the total energy (which represents the eigenvalue) and $V(x)$ the potential energy distribution; $\hbar \equiv h / 2 \pi, h(=6.626$ $x 10^{-34} \mathrm{~J}-\mathrm{s}$ ) is Planck's constant.

Often Eq. (2) is written as

$$
H \psi=E \psi,
$$

where 


$$
H=\frac{p^{2}}{2 m}+V(x)=-\frac{\hbar^{2}}{2 m} \frac{d^{2}}{d x^{2}}+V(x)
$$

represents the Hamiltonian of the problem and $p$ is the $x$ component of the momentum that, in quantum mechanics, becomes the linear operator $-i \hbar d / d x$. The application of the boundary conditions leads to the eigenvalue equation, as will be discussed below.

The linear harmonic oscillator is one of the most important problems in quantum mechanics. Its potential energy distribution is characterized by

$$
V(x)=\frac{1}{2} m \omega^{2} x^{2},
$$

where $\omega$ is the "classical frequency" of the oscillator. Substituting Eq. (5) into Eq. (2), we obtain

$$
\frac{d^{2} \psi}{d x^{2}}+\frac{2 m}{\hbar^{2}}\left[E-\frac{1}{2} m \omega^{2} x^{2}\right] \Psi(x)=0
$$

and

$$
\frac{d^{2} \Psi}{d \xi^{2}}+\left(\Lambda-\xi^{2}\right) \Psi(\xi)=0,
$$

where

$$
\xi(x)=\alpha x=\left(\frac{m \omega}{\hbar}\right)^{1 / 2} x,
$$

and 


$$
\Lambda=\frac{2 E}{\hbar \omega} \text {. }
$$

If we apply the boundary conditions that $\Psi(\xi)$ should approach 0 as $\xi \rightarrow \pm \infty$, we find that [see, for example, Schift (1968)]:

$$
\Lambda=(2 n+1) ; \quad n=0,1,2, \ldots
$$

These are the eigenvalues for the problem. Only if $\Lambda=1,3,5$, $7, \ldots$, will the corresponding $\Psi(\xi)$ approach 0 as $\xi \rightarrow \pm \infty$; indeed, for $\Lambda \neq=1,3,5,7, \ldots, \Psi(\xi)$ tends to $\pm \infty$ as $\xi$ becomes large without limit. (We shall show this in example 5.1; see Figs. 5.5 and 5.6.) The corresponding orthonormal eigenfunctions are

$$
\Psi_{n}(x)=N_{n} H_{n}(\xi) \exp \left(-\frac{1}{2} \xi^{2}\right) ; \quad n=0,1,2, \ldots,
$$

where

$$
N_{n}=\left(\frac{\alpha}{\pi^{1 / 2} 2^{n} n !}\right)^{1 / 2}
$$

represents the normalization constant and $H_{n}(\xi)$ are the Hermite polynomials, 


$$
\begin{array}{r}
H_{0}(\xi)=1 ; H_{1}(\xi)=2 \xi ; H_{2}(\xi)=4 \xi^{2}-2 ; \\
H_{3}(\xi)=8 \xi^{3}-12 \xi ; H_{4}(\xi)=16 \xi^{4}-48 \xi^{2}+12 ;
\end{array}
$$

Higher-order Hermite polynomials can be obtained from the recurrence relations

$$
H_{n+1}=2 x H_{n}(x)-2 n H_{n-1}(x) \text {. }
$$

The functions $\Psi_{n}(x)$ form a complete set, and

$$
\int_{-\infty}^{+\infty} \Psi_{m}^{*}(x) \Psi_{n}(x) d x=\delta_{m n} .
$$

Before considering the WKB solutions, we mention here that in describing the propagation of electromagnetic waves in a slab waveguide having a nonuniform refractive index distribution $n(x)$ the solution of Maxwell's equation for TE modes is given by

$$
E_{y}(x, z, t)=\psi(x) \exp [i(\omega t-\beta z)],
$$

where $\mathrm{E}_{\mathrm{y}}$ represents the electric field, $\omega$ the angular frequency, and $\beta$ the propagation constant. The field $\Psi(x)$ satisfies the equation [Ghatak and Thyagarajan (1989)]

$$
\frac{d^{2} \Psi(x)}{d x^{2}}+\left[k_{0}^{2} n^{2}(x)-\beta^{2}\right] \psi(x)=0,
$$


where $k_{0}=\omega / c$ is the free-space wave number. Equation (16) is in the form of Eq. (1) with

$$
\Gamma^{2}(x)=k_{0}^{2} n^{2}(x)-\beta^{2} .
$$

The propagation constant $(\beta)$ represents the eigenvalue, the determination of which is of fundamental importance in waveguide theory. For an untruncated parabolic index,

$$
n^{2}(x)=n_{1}^{2}\left[1-2 \Delta\left(\frac{x}{a}\right)^{2}\right],
$$

where $n_{1}$ is the refractive index on the axis (at $x=0$ ), $\Delta$ and $a$ are constants.

The infinitely extended (or untruncated) profile of Eq. (18) is a physical and a practical impossibility. In practice, Eq. (18) is valid only for $|x|<a$, that is, inside the core. The parabolic profile is, in this sense, truncated. Outside the core region, the refractive index is normally constant.

The untruncated parabolic profile of Eq. (18) is of practical interest in spite of its physical impossibility. Its practical interest comes about because it yields an exact solution and can therefore provide guidance when the truncated counterpart "looks" untruncated. This happens, for example, when the mode in question is tightly bound to the core, that is, when most of the modal energy is in the core. This, in turn, happens when the mode is well beyond cutoff. Under these conditions, guidance is 
influenced almost exclusively by the refractive index in the core. The approximate solutions (and eigenvalues) of the truncated profile should begin to replicate the exact solutions (and eigenvalues) of the untruncated profile as this tightly bound condition is approached.

For $n^{2}(x)$ given by Eq. (18), Eq. (16) can be transformed to the form of Eq. (7) with

$$
\left.\begin{array}{l}
\alpha=\left(\frac{k_{0}^{2} n_{1}^{2} 2 \Delta}{a^{2}}\right)^{1 / 4} \\
\Lambda=\frac{k_{0}^{2} n_{1}^{2}-\beta^{2}}{k_{0} n_{1} \sqrt{2 \Delta} / a}
\end{array}\right\} .
$$

Therefore, the eigenvalue equation of the type given by Eq. (2) or Eq. (16) should be of profound importance in physics and engineering. For the parabolic profile of Eqs. (5) and (18), analytical solutions are possible; however, in general one must resort to numerical or approximate methods. One of the most used approximations is the WKB method, which will be discussed in this chapter. In the Chapter 6 , we will discuss the method that uses Airy functions. As discussed in Chapter 2, the WKB solutions are not valid at or near the turning points; on the other hand, the MAF solutions remain accurate for all values of $x$. 


\subsection{The WKB Quantization Condition}

We consider first a general $\Gamma^{2}(x)$ variation of the type shown in Fig. 5.1. For a bound state in quantum mechanics or for a guided mode in optical planar waveguide theory, the wave function $\Psi(x)$ must decay exponentially as $x \rightarrow \pm \infty$. Therefore, $\Gamma^{2}(x)$ must be negative as $x$ tends to $\pm \infty$. A typical behavior is shown in Fig. 5.1; the points $x=x_{1}$, and $x=x_{2}$ represent the turning points where $\Gamma^{2}(x)=0$. For the harmonic oscillator problem [see Eq. (6)], $\Gamma^{2}(x)$ is an inverted symmetric parabola with

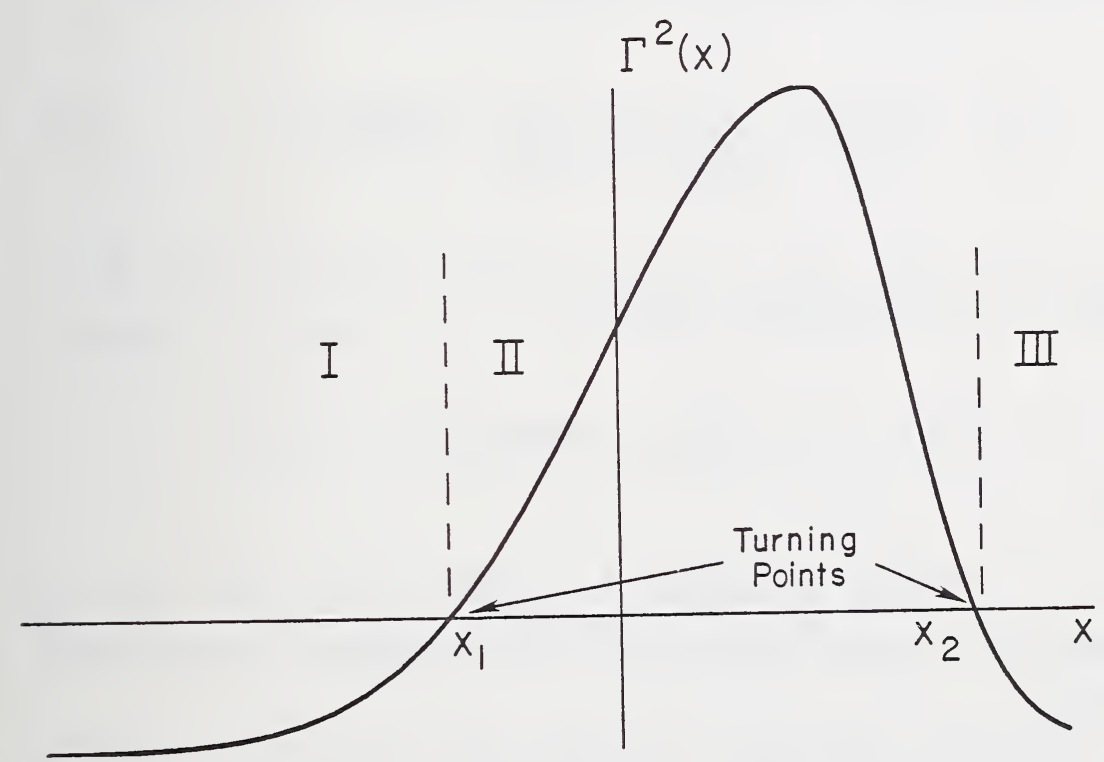

Figure 5.1 A typical variation of $\Gamma^{2}(x)$. 


$$
x_{2}=-x_{1}=\sqrt{\frac{2 E}{m \omega^{2}}} .
$$

Similarly, for the waveguide problem, for $x<x_{1}$ (Region I) and for $x>x_{2}$ (Region III) the solutions must be exponentially decaying. We start from Region I and use the connection formulas to determine the solution in Region III and obtain the condition for which the coefficient of the exponentially increasing solution in Region III is 0 .

The exponentially decaying solution in Region I is given by

$$
\Psi_{I}=\frac{A}{\sqrt{\kappa(x)}} \exp \left[-\int_{x}^{x_{1}} \kappa(x) d x\right]
$$

where, as in the previous chapter,

$$
\kappa^{2}(x)=-\Gamma^{2}(x),
$$

which is positive in Regions I and III. If we now use the connection formula given by Eq. (53) of Chapter 2, the solution becomes

$$
\Psi_{I I}=\frac{2 A}{\sqrt{\Gamma(x)}} \sin \left[\int_{x_{1}}^{x} \Gamma(x) d x+\pi / 4\right] .
$$

To obtain $\Psi_{\text {III }}$, we must "look" toward the turning point $x=x_{2}$. To do so, we write 


$$
\begin{aligned}
\int_{x_{1}}^{x} \Gamma d x+\pi / 4 & =\int_{x_{1}}^{x_{2}} \Gamma(x) d x-\int_{x}^{x_{2}} \Gamma(x) d x+\pi / 4 \\
& =\frac{\pi}{2}+\theta-\left(\int_{x}^{x_{2}} \Gamma(x) d x+\pi / 4\right),
\end{aligned}
$$

where

$$
\theta \equiv \int_{x_{1}}^{x_{2}} \Gamma(x) d x
$$

Substituting Eq. (23) into Eq. (22) and simplifying, we obtain

$$
\begin{aligned}
\Psi_{I I}(x) & =(2 A \cos \theta) \frac{1}{\sqrt{\Gamma(x)}} \cos \left[\int_{x}^{x_{2}} \Gamma(x) d x+\frac{\pi}{4}\right] \\
+ & (2 A \sin \theta) \frac{1}{\sqrt{\Gamma(x)}} \sin \left[\int_{x}^{x_{2}} \Gamma(x) d x+\frac{\pi}{4}\right] .
\end{aligned}
$$

Using the connection formulas given by Eqs. (46) and (47) of Chapter 2, we find

$$
\begin{aligned}
\psi_{I I I}(x)= & 2 A \cos \theta \frac{1}{\sqrt{\kappa}} \exp \left[+\int_{x_{2}}^{x} \kappa(x) d x\right] \\
& +A \sin \theta \frac{1}{\sqrt{\kappa}} \exp \left[-\int_{x_{2}}^{x} \kappa(x) d x\right] .
\end{aligned}
$$

The first term on the right side of this equation represents an exponentially growing solution that should not be present; we therefore require

$$
\cos \theta=0 \Rightarrow \theta=\left(n+\frac{1}{2}\right) \pi
$$




$$
\theta=\int_{x_{1}}^{x_{2}} \Gamma(x) d x=\left(n+\frac{1}{2}\right) \pi ; \quad n=0,1,2, \ldots,
$$

which represents the quantization condition. After finding the eigenvalues [from Eq. (26)], $\Gamma(x)$ is completely known and therefore the complete solution is given by

$$
\Psi(x)=\left\{\begin{array}{ccc}
\frac{A}{\sqrt{\kappa}(x)} & \exp \left[-\int_{x}^{x_{1}} \kappa(x) d x\right], & x<x_{1}, \\
\frac{2 A}{\sqrt{\Gamma}(x)} & \sin \left[\int_{x_{1}}^{x} \Gamma(x) d x+\frac{\pi}{4}\right], & x_{1}<x<x_{2}, \\
\frac{2 A}{\sqrt{\Gamma(x)}}(-1)^{n} & \sin \left[\int_{x}^{x_{2}} \Gamma(x) d x+\frac{\pi}{4}\right], & x_{1}<x<x_{2}, \\
\frac{A(-1)^{n}}{\sqrt{\kappa(x)}} & \exp \left[-\int_{x_{2}}^{x} \kappa(x) d x\right], & x>x_{2} .
\end{array}\right.
$$

These equations are simple to use. They also readily show the importance of using the correct eigenvalue. If the eigenvalue does not satisfy Eq. (26), the solution will diverge in the region $x>x_{2}$. This is the principle upon which most numerical methods are based. We choose an eigenvalue and study the behavior of the solution at large values of $x$; if it starts diverging, another value is chosen and the process continues thus until the solution goes to zero as $x \rightarrow \infty$. We will show this explicitly through the following example. 


\subsection{Examples}

\section{Example 5.1}

We consider the linear harmonic oscillator, for which

$$
\Gamma^{2}(x)=\Lambda-x^{2}=\lambda^{2}-x^{2},
$$

[see Eq. (7)] where $\lambda^{2} \equiv \Lambda$. The turning points are given by

$$
x_{2}=-x_{1}=\lambda \text {, }
$$

and Eq. (26) becomes

$$
\int_{-\lambda}^{+\lambda} \sqrt{\lambda^{2}-x^{2}} d x=\left(n+\frac{1}{2}\right) \pi
$$

If we use the substitution $x=\lambda \sin \theta$, we readily get

$$
\lambda^{2}\left[\frac{\theta}{2}+\frac{1}{4} \sin 2 \theta\right]_{-\pi / 2}^{+\pi / 2}=\left(n+\frac{1}{2}\right) \pi
$$

or

$$
\Lambda=\lambda^{2}=2 n+1,
$$

which incidentally represents the exact eigenvalues of the problem [see Eq. (10)]. This exact eigenvalue is, at once, interesting and 
puzzling. We do not know why the $W K B$ method should yield exact results.

As a by-product of this happenstance, we expect the WKB formalism to yield highly accurate eigenvalues for a truncated parabolic profile, provided that the mode in question is bound tightly to the core region of the waveguide, where the refractive index varies in a parabolic fashion. The modes are tightly bound if they are far from cutoff (that is, high refractive index contrast, large core, or short wavelength).

To determine the eigenfunctions, we note that since $\Gamma^{2}(-x)=\Gamma^{2}(x)$, the eigenfunctions must be either symmetric or antisymmetric, that is, either

$$
\left.\begin{array}{lcc}
\psi(-x)=\psi(x), & \text { symmetric } \\
\psi(-x)=-\Psi(x), & \text { antisymmetric }
\end{array}\right\} .
$$

We consider only the antisymmetric case for which the WKB solution in the region $0<x<\lambda$ is given by

$$
\Psi_{I I}(x)=\frac{1}{\sqrt{\lambda}} \cdot \frac{1}{\sqrt{\Gamma(x)}} \sin \left[\int_{0}^{x} \Gamma(x) d x\right]
$$

where we have chosen the multiplicative constant so that $\Psi^{\prime}(0)=1$. To use the connection formula, we write Eq. (32) as 


$$
\begin{aligned}
\Psi_{I I}(x)= & \frac{1}{\sqrt{\lambda} \sqrt{\Gamma(x)}} \sin \left[\alpha-\left(\int_{x}^{\lambda} \Gamma(x) d x+\frac{\pi}{4}\right)\right] \\
& =\frac{1}{\sqrt{\lambda} \sqrt{\Gamma(x)}}\left[\sin \alpha \cos \left(\eta(x)+\frac{\pi}{4}\right)\right. \\
& \left.-\cos \alpha \sin \left(\eta(x)+\frac{\pi}{4}\right)\right],
\end{aligned}
$$

where

$$
\left.\begin{array}{ccc}
\alpha & =\int_{0}^{\lambda} \Gamma(x) d x+\frac{\pi}{4}=\eta(0)+\frac{\pi}{4}, \\
\eta(x) & = & \int_{x}^{\lambda} \Gamma(x) d x
\end{array}\right\}
$$

Thus, in Region III (see Fig. 5.1) we have

$$
\begin{aligned}
\Psi_{I I I}(x) & =\frac{1}{\sqrt{\lambda} \sqrt{\kappa(x)}}[\sin \alpha \exp [\zeta(x)] \\
& \left.-\frac{1}{2} \cos \alpha \exp [-\zeta(x)]\right]
\end{aligned}
$$

where

$$
\zeta(x)=\int_{\lambda}^{x} \kappa(x) d x .
$$


For $\Gamma^{2}(x)$ given by Eq. (28), the integrals can be evaluated to obtain

$$
\begin{aligned}
& \eta(x)=\int_{x}^{\lambda} \sqrt{\lambda^{2}-x^{2}} d x=\frac{\pi \lambda^{2}}{4} \\
& -\frac{x}{2} \sqrt{\lambda^{2}-x^{2}}-\frac{\lambda^{2}}{2} \sin ^{-1}\left(\frac{x}{\lambda}\right)
\end{aligned}
$$

and

$$
\begin{aligned}
\zeta(x)= & \int_{\lambda}^{x} \sqrt{x^{2}-\lambda^{2}} d x=\frac{x}{2} \sqrt{x^{2}-\lambda^{2}} \\
& -\frac{1}{2} \lambda^{2} \ln \left|\frac{x+\sqrt{x^{2}-\lambda^{2}}}{\lambda}\right| .
\end{aligned}
$$

The WKB wave functions are now known. The exact wavefunctions are

$$
\begin{gathered}
\Psi_{E X A C T}=\frac{1}{2}(-1)^{(n-1) / 2} \frac{\left(\frac{n-1}{2}\right) !}{n !} H_{n}(x) \exp \left(-\frac{x^{2}}{2}\right), \\
n=1,3,5, \ldots,
\end{gathered}
$$

where we have chosen the multiplicative constant so that $\Psi^{\prime}(0)=1$. In Figs. 5.2, 5.3, and 5.4 we have plotted the exact (viz. the Hermite-Gauss functions) and the WKB wavefunctions for $\Lambda=3,7$, and 11 .

The WKB solutions obviously fail near the turning points $(x= \pm \sqrt{ } 3$ for $\Lambda=3, x= \pm \sqrt{ } 7$ for $\Lambda=7$, and $x= \pm \sqrt{ } 11$ for $\Lambda=11)$. The two solutions are in good agreement for $x$ not close 


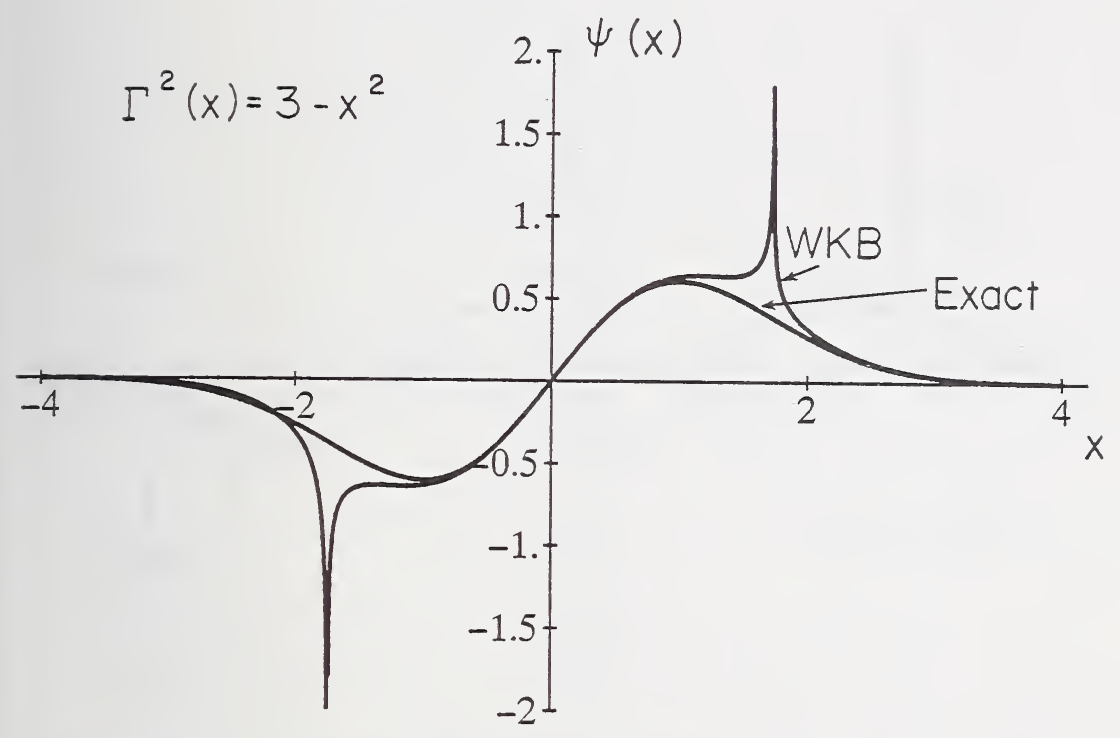

Figure 5.2 Exact and $W K B$ wavefunctions for $\Lambda=3(\mathrm{n}=1)$, which corresponds to $\alpha=\pi$. $\Gamma^{2}(x)=3-x^{2}$.

to the turning points. In Figs. 5.5 and 5.6 we show how the large $|x|$ behavior changes significantly if we use a value of $\Lambda$ that is slightly different from the correct eigenvalue. These figures illustrate the sensitivity of the solution to the eigenvalue. Even a small error in the eigenvalue causes a dramatic change in the behvaior of the wavefunction. The large deviation is due to the exponential term in the WKB solution. A slight error has profound consequences. The figures also show the qualitative improvement in the WKB solutions for increasing mode number (that is, increasing values of $\Lambda$ ).

Problem 5.1. In continuation of Example 5.1, obtain the WKB eigenfunctions for the symmetric case and compare with the exact 


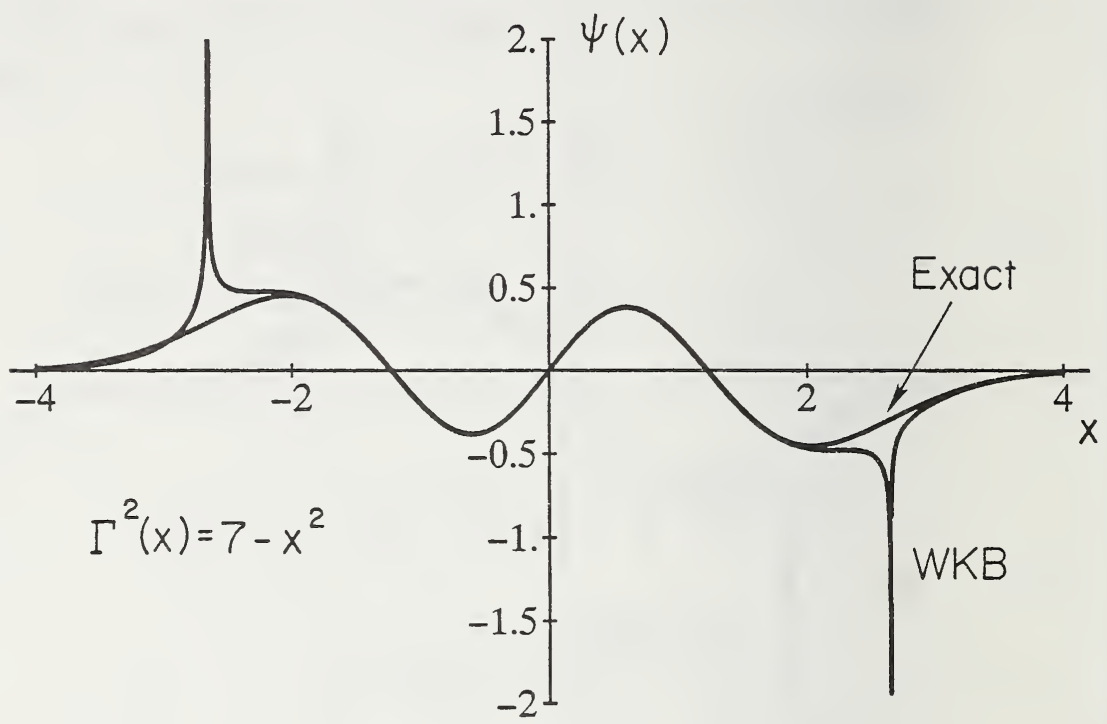

Figure 5.3 Exact and $W K B$ wavefunctions for $\Lambda=7(\mathrm{n}=3)$, which corresponds to $\alpha=2 \pi \cdot \Gamma^{2}(x)=7-x^{2}$.

eigenfunction given by Eq. (11) with $n=0,2$, and 4 .

Problem 5.2. Use the connection formulas to show that the WKB wavefunction in the region $x<-\lambda$ is given by [cf. Eq. (35)]

$$
\begin{gathered}
\psi(x)=\frac{1}{\sqrt{\lambda} \sqrt{\kappa(x)}}\left[\frac{1}{2} \cos \alpha\right. \\
\cdot \exp [-\zeta(x)]-\sin \alpha \exp [+\zeta(x)]]
\end{gathered}
$$

where

$$
\zeta(x)=\int_{x}^{-\lambda} \kappa(x) d x .
$$




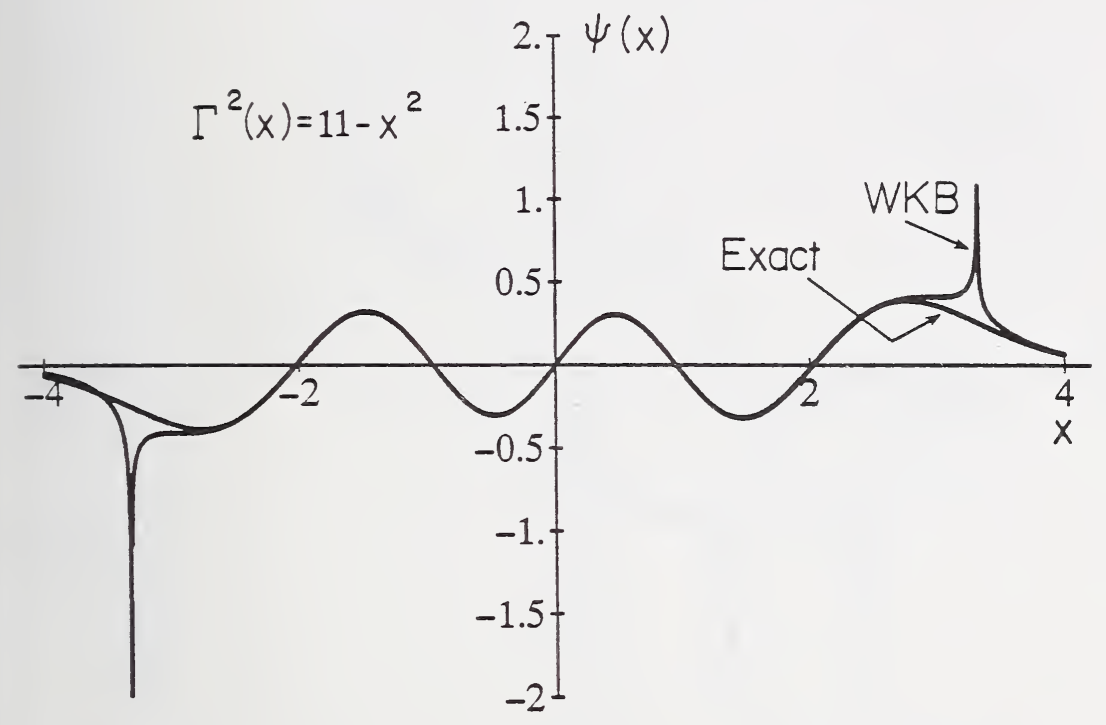

Figure 5.4 Exact and $W K B$ wavefunctions for $\Lambda=11(\mathrm{n}=5)$, which corresponds to $\alpha=3 \pi$. $\Gamma^{2}(x)=11-x^{2}$.

\section{Example 5.2}

We next consider the asymmetric profile

$$
\Gamma^{2}(x)=f(x)-\lambda,
$$

where 


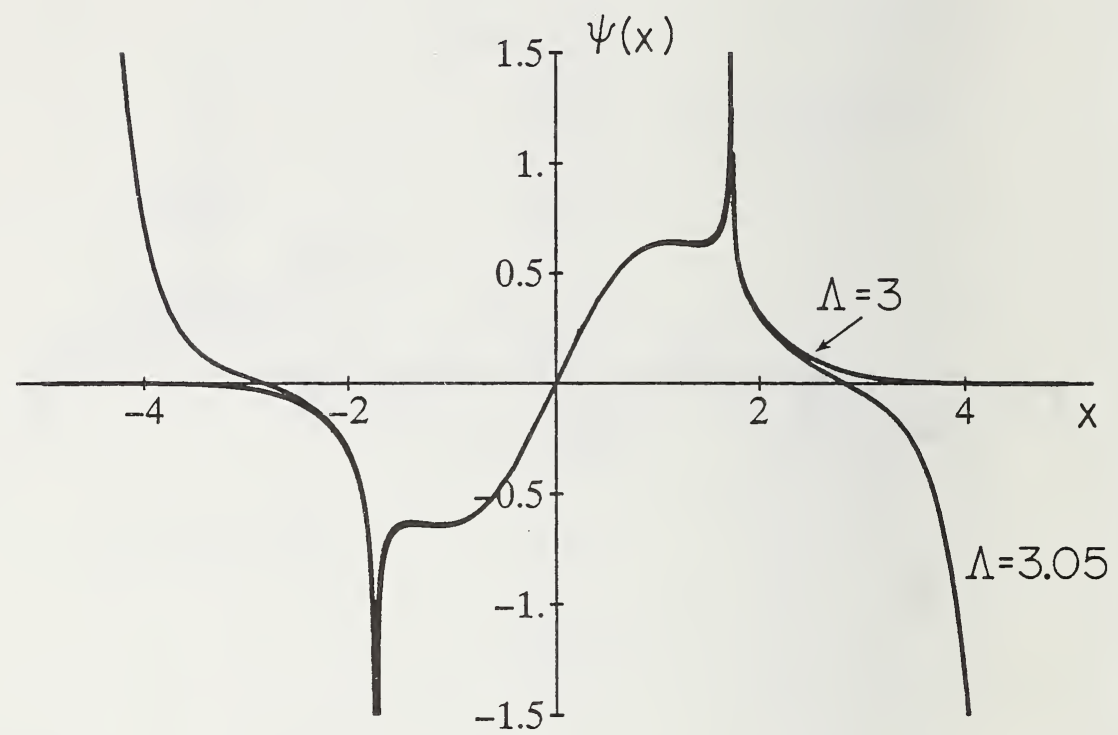

Figure 5.5 $W K B$ solutions for $\Lambda=3$ and $\Lambda=3.05$. For $\Lambda=$ 3.05 , the coefficient of the exponential term is not zero, causing the wavefunction to flare up for $|x|$ $>\sqrt{3}$.

$$
f(x)= \begin{cases}f_{1}(x)=a+b+\frac{b}{2 d} x, & x<0 \\ f_{2}(x)=a+b \exp \left(-\frac{x}{d}\right), & x>0 .\end{cases}
$$

$a, b$, and $d$ are constants and $\lambda$ is the eigenvalue. The exact solution for this profile is 


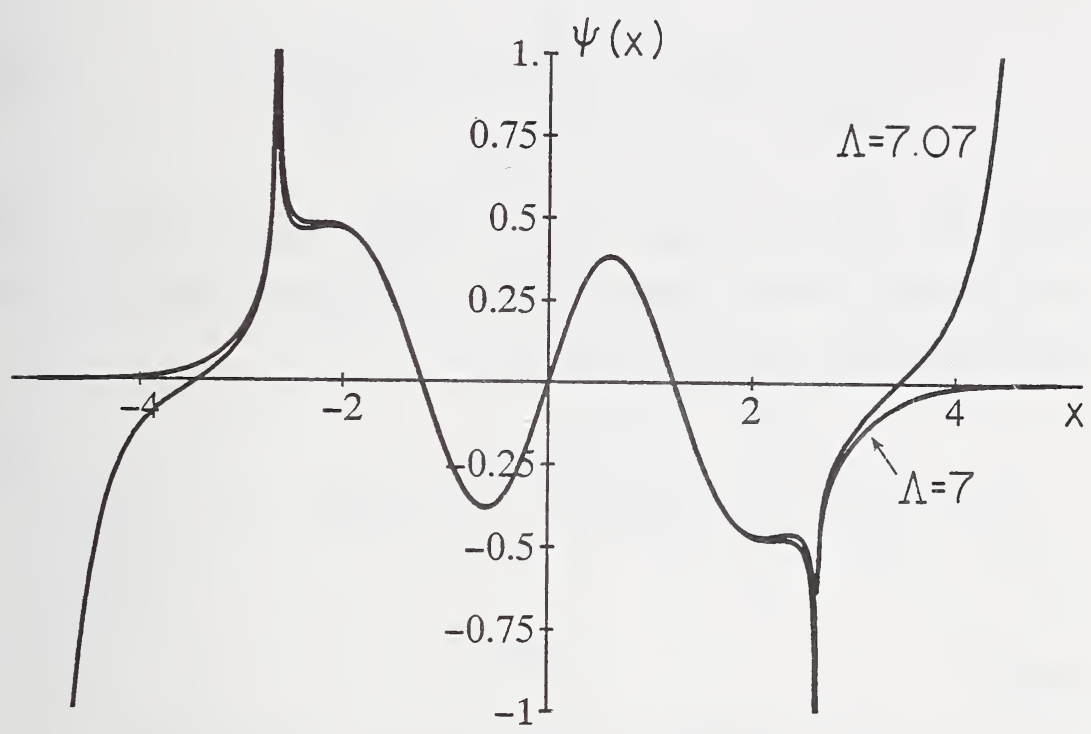

Figure 5.6 WKB solutions for $\Lambda=7$ and $\Lambda=7.07$. For $\Lambda=$ 7.07 the coefficient of the exponential term is not zero resulting in the flaring up of the wavefunction for $|x|>\sqrt{7}$.

$$
\Psi_{E X A C T}(x)= \begin{cases}\frac{A i\left(z_{1}-b_{1}^{1 / 3} x\right)}{A i\left(z_{1}\right)}, & x<0 \\ \frac{J_{v}\left[z_{2} \exp \left(-\frac{x}{2 d}\right)\right]}{J_{v}\left(z_{2}\right)}, & x>0,\end{cases}
$$

where $a_{1}=a+b-\lambda, b_{1}=b / 2 d, v=2 d(\lambda-a)^{1 / 2}, z_{1}=-$ $a_{1} /\left(b_{1}\right)^{2 / 3}, z_{2}=2 d(b)^{1 / 2}$, and the multiplying constant has been chosen to ensure that $\Psi_{\text {EXACT }}(0)=1$. Continuity of $\Psi$ and $\Psi^{\prime}$ at $x=0$ yields 


$$
\frac{A i^{\prime}\left(z_{1}\right)}{A i\left(z_{1}\right)}\left(b_{1}\right)^{1 / 3}=\frac{\sqrt{b}}{2} \frac{J_{v-1}\left(z_{2}\right)-J_{v+1}\left(z_{2}\right)}{J_{v}\left(z_{2}\right)} .
$$

From Eq. (42), we can find the (exact) eigenvalues. The corresponding (exact) eigenfunctions are found from Eq. (41). The WKB eigenvalues are found from

$$
\int_{x_{1}}^{x_{2}} \Gamma(x) d x=\int_{x_{1}}^{0} \Gamma_{1}(x) d x+\int_{0}^{x_{2}} \Gamma_{2}(x) d x=\left(m+\frac{1}{2}\right) \pi,
$$

where

$$
\begin{array}{rlr}
\Gamma^{2}(x) & =\Gamma_{1}^{2}(x)=f_{1}(x)-\lambda \quad \text { for } x<0 \\
& =\Gamma_{2}^{2}(x)=f_{2}(x)-\lambda \quad \text { for } x>0,
\end{array}
$$

and $x_{1}$ and $x_{2}$ are again the two turning points, $\Gamma_{1}\left(x_{1}\right)=0=\Gamma_{2}\left(x_{2}\right)$. For $\Gamma^{2}(x)$ given by Eqs. (39) and (40), Eq. (43) simplifies to

$$
\frac{2}{3 b_{1}} a_{1}^{3 / 2}+2 d\left[\sqrt{a_{1}}-\sqrt{\lambda-a} \tan ^{-1}\left(\frac{a_{1}}{\lambda-a}\right)^{1 / 2}\right]=\left(m+\frac{1}{2}\right) \pi \text {. }
$$

We have used typical waveguide parameters in the profile of Eq. (40) and found the eigenvalues. We used $a=4, b=0.2$, and $d=10$. Figure 5.7(a) shows a plot of $f(x)$, and Fig. 5.7(b) shows $\Gamma^{2}(x)$ for $\lambda=4.14$; this is the approximate value of the first eigenvalue. Indeed, $\lambda$ for the first two modes is 


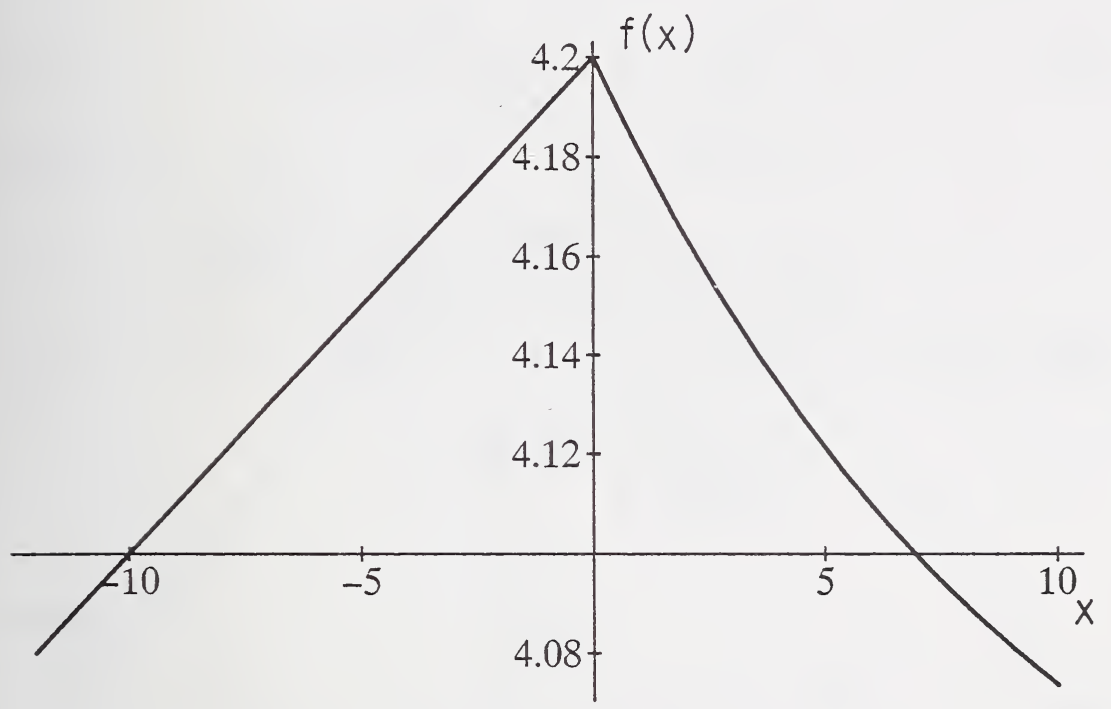

Figure 5.7(a) Variation of $\mathrm{f}(x)$ for $a=4, b=0.2$ and $d=10$.

$\begin{array}{lcc}\lambda(\mathrm{n}=0) & \text { EXACT } & \text { WKB } \\ \lambda(\mathrm{n}=1) & 4.079276 & 4.139262 \\ & 4.079617\end{array}$

The corresponding $W K B$ solutions are readily obtained. For $x<x_{1}$ :

$$
\psi(x)=\frac{A}{2 \sqrt{\kappa_{1}(x)}} \exp \left[-\frac{4 d}{3 b} \kappa_{1}^{3}(x)\right],
$$

where 


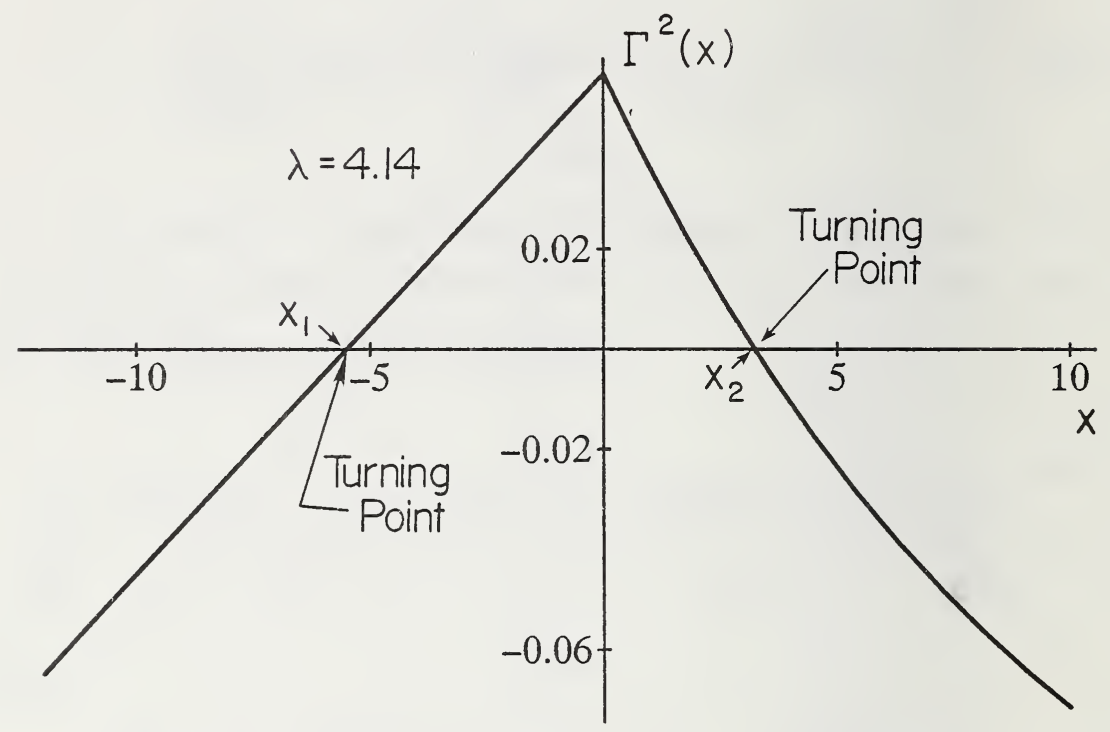

Figure 5.7(b) The variation of $\Gamma^{2}(x)$ for $\lambda=4.14$.

$$
\kappa_{1}(x)=\left[\lambda-a-b-\frac{b}{2 d} x\right]^{1 / 2} .
$$

For $x_{1}<x<0$ :

$$
\Psi(x)=\frac{A}{\sqrt{\Gamma_{1}(x)}} \sin \left[\frac{4 d}{3 b} \Gamma_{1}^{3}(x)+\frac{\pi}{4}\right],
$$

where 


$$
\Gamma_{1}(x)=\left[a+b-\lambda+\frac{b x}{2 d}\right]^{1 / 2} .
$$

For $0<x<x_{2}$ :

$$
\begin{gathered}
\Psi(x)=\frac{(-1)^{m} A}{\sqrt{\Gamma_{2}(x)}} \sin \left[\frac{\pi}{4}+2 d\left\{\Gamma_{2}(x)\right.\right. \\
\left.\left.-\alpha \tan ^{-1} \frac{\Gamma_{2}(x)}{\alpha}\right\}\right],
\end{gathered}
$$

where

$$
\begin{gathered}
\Gamma_{2}(x)=\left[a+b \exp \left(-\frac{x}{d}\right)-\lambda\right]^{1 / 2}, \\
\alpha=\sqrt{\lambda-a} .
\end{gathered}
$$

For $x>x_{2}$ :

$$
\begin{gathered}
\Psi(x)=\frac{(-1)^{m} A}{2 \sqrt{k_{2}(x)}} \\
\exp \left[-2 d\left(\frac{\alpha}{2} \ln \left|\frac{\alpha+k_{2}(x)}{\alpha-k_{2}(x)}\right|-k_{2}(x)\right)\right],
\end{gathered}
$$

where$$
\kappa_{2}(x)=\left[\lambda-a-b \exp \left(-\frac{x}{d}\right)\right]^{1 / 2}
$$

In writing Eq. (48a), we have made use of the eigenvalue Eq. (43). The value of $A$ 


$$
A=\frac{(a+b-\lambda)^{1 / 4}}{\sin \left[\frac{4 d}{3 b}(a+b-\lambda)^{3 / 2}+\frac{\pi}{4}\right]}
$$

is determined from the condition $\Psi(0)=1$. Figures 5.8 and 5.9 show the exact and the WKB solutions for the first two modes for the profile shown in Fig. 5.7b.

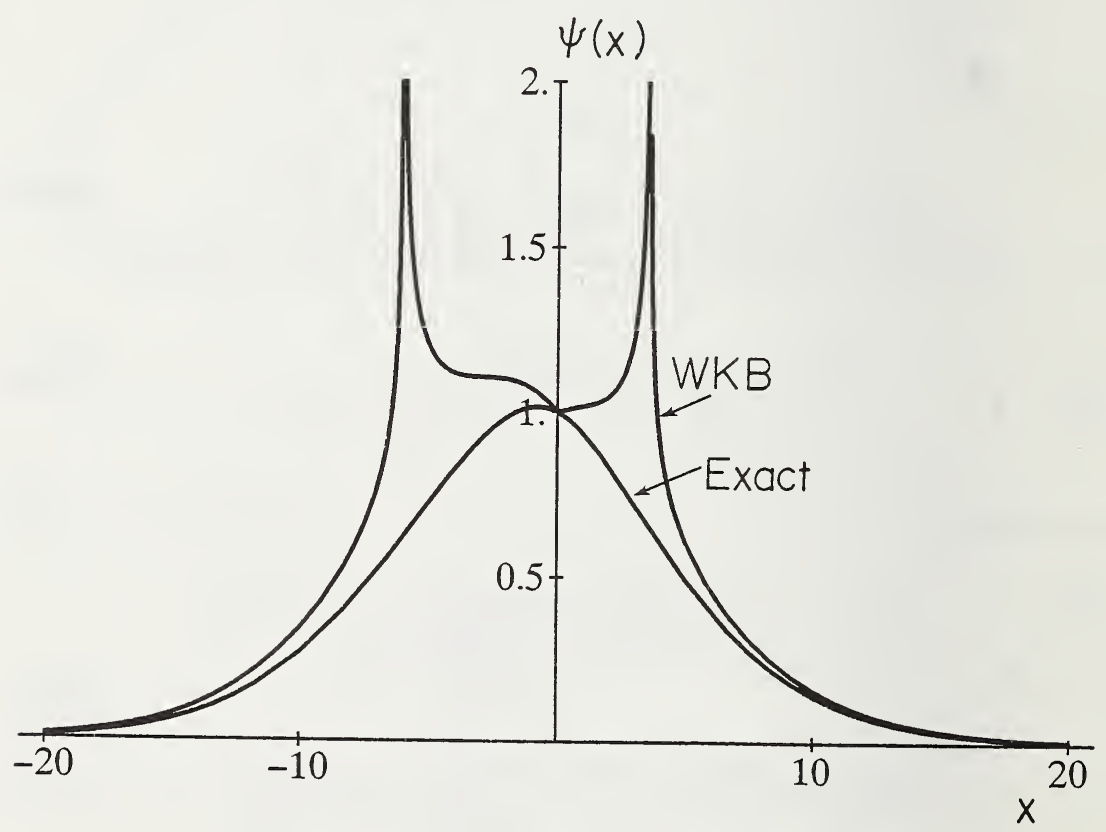

Figure 5.8 The exact and $W K B$ eigenfunctions corresponding to the first mode for the profile shown in Fig. 5.7. 


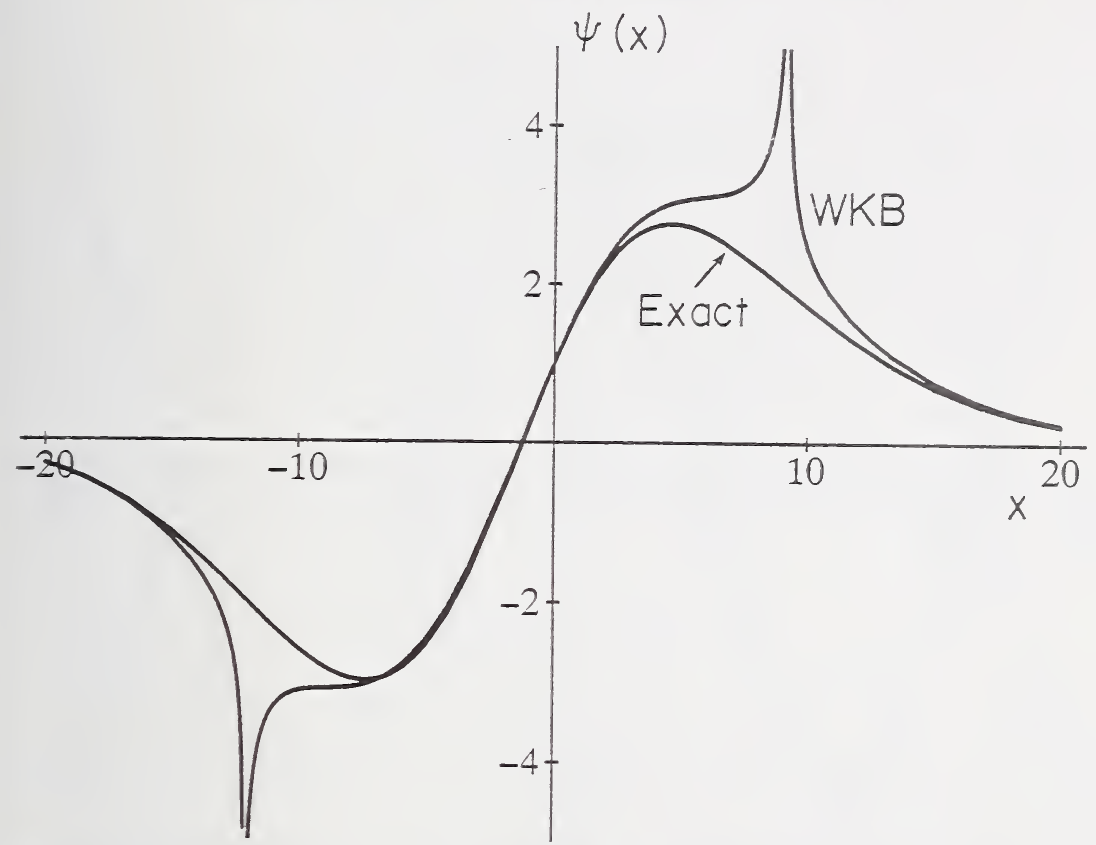

Figure 5.9 The exact and WKB eigenfunctions corresponding to the second mode for the profile shown in Fig. 5.7.

\section{Example 5.3}

We next illustrate the use of the WKB method for solving the Schrödinger equation [see Eq. (2)] corresponding to the potential energy distribution shown in Fig. 5.10(a). For simplicity, we assume $V(x)$ to be symmetric in $x[V(-x)=V(x)]$; this immediately implies that the eigenfunctions are either symmetric or antisymmetric in $x$ :

$$
\psi(-x)=\psi(x) \text { (symmetric) } \Rightarrow \psi^{\prime}(0)=0,
$$




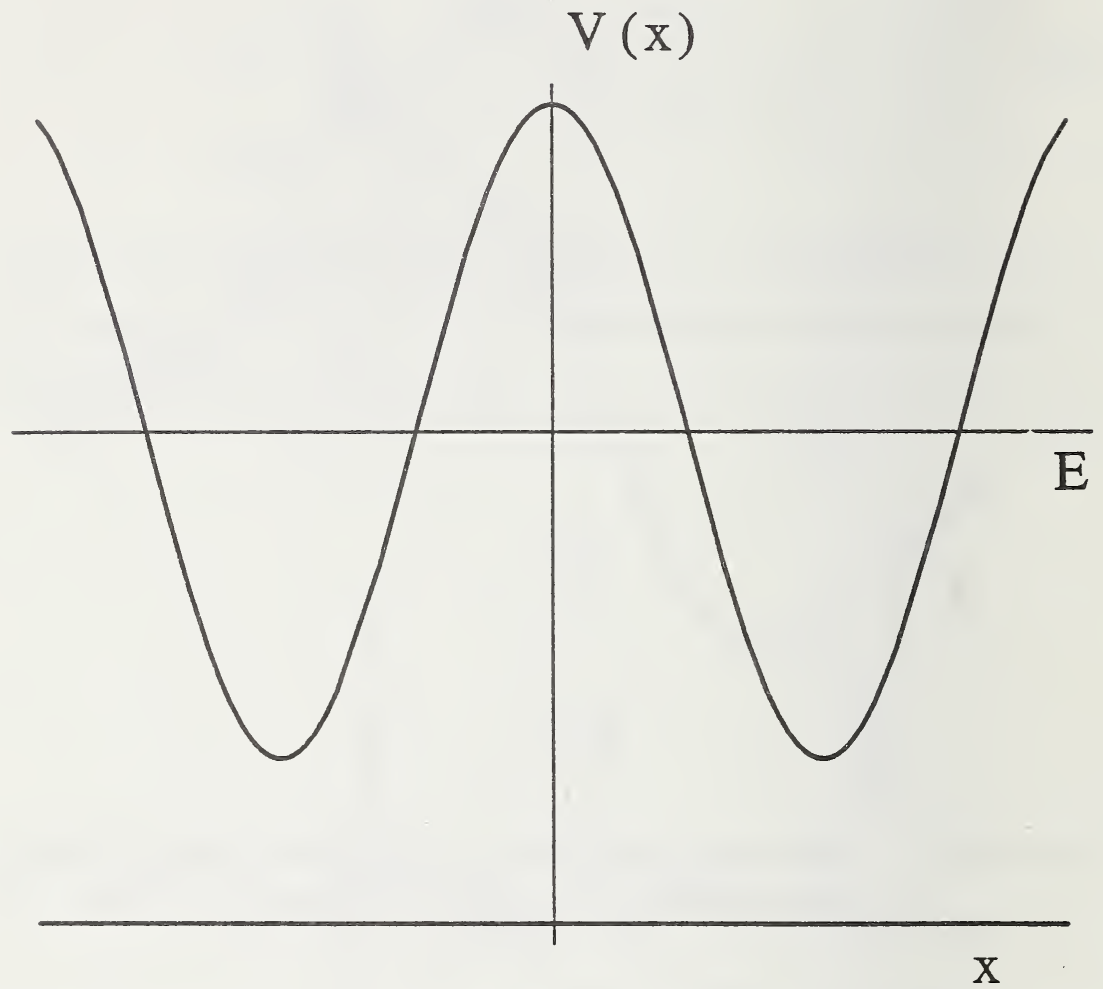

Figure 5.10(a) Potential energy variation having two wells.

$$
\psi(-x)=-\psi(x) \text { (antisymmetric) } \Rightarrow \psi(0)=0 .
$$

The energy eigenvalue $E$ is to be determined. The variation of $\Gamma^{2}(x)$ is shown in Fig. 5.10(b); there are now four turning points. A bound state must correspond to an exponentially decreasing in Region V. Thus,

$$
\Psi_{V}(x)=\frac{A}{\sqrt{\kappa(x)}} \exp \left[-\int_{x_{2}}^{x} \kappa(x) d x\right] .
$$

Using the connection formula [see Eq. (46) of Chapter 2], we get 


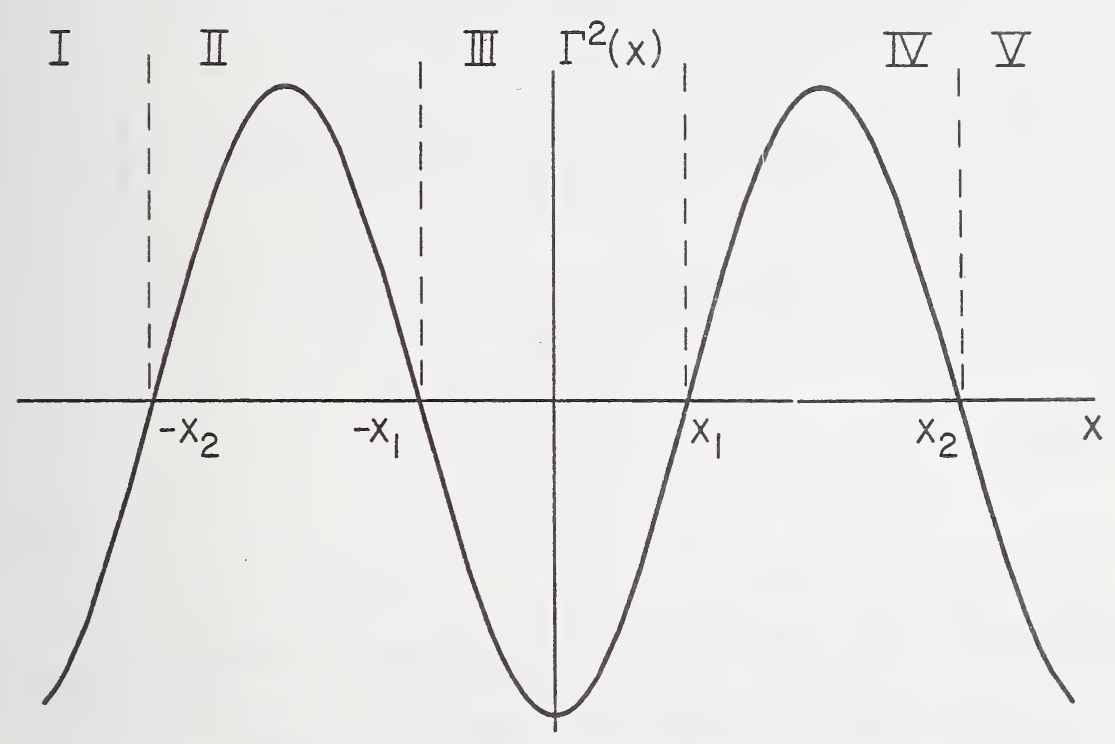

Figure 5.10(b) The corresponding variation of $\Gamma^{2}(x)$.

$$
\Psi_{I V}(x)=\frac{2 A}{\sqrt{\Gamma(x)}} \sin \left[\int_{x}^{x_{2}} \Gamma(x) d x+\frac{\pi}{4}\right]
$$

To get to Region III, we must "look" toward the turning point $x=x_{1}$ : 


$$
\begin{aligned}
\Psi_{I V}(x) & =\frac{2 A}{\sqrt{\Gamma(x)}} \cos \left[\theta-\left(\int_{x_{1}}^{x} \Gamma(x) d x+\frac{\pi}{4}\right)\right] \\
& =2 A \cos \theta \frac{1}{\sqrt{\Gamma(x)}} \cos \left(\int_{x_{1}}^{x} \Gamma(x) d x+\frac{\pi}{4}\right) \\
& +2 A \sin \theta \frac{1}{\sqrt{\Gamma(x)}} \sin \left(\int_{x_{1}}^{x} \Gamma(x)+\frac{\pi}{4}\right),
\end{aligned}
$$

where

$$
\theta=\int_{x_{1}}^{x_{2}} \Gamma(x) d x .
$$

We now use Eqs. (53) and (54) of Chapter 2 to obtain

$$
\begin{aligned}
\Psi_{I I I}(x) & =2 A \cos \theta \frac{1}{\sqrt{\kappa}} \exp \left[\int_{x}^{x_{1}} \kappa(x) d x\right] \\
& +A \sin \theta \frac{1}{\sqrt{\kappa}} \exp \left[-\int_{x}^{x_{1}} \kappa(x) d x\right] .
\end{aligned}
$$

Applying the conditions given by Eqs. (51) and (52), we obtain

$$
\cot \left[\int_{x_{1}}^{x_{2}} \Gamma(x) d x\right]= \pm \frac{1}{2} \exp \left[-2 \int_{0}^{x_{1}} \kappa(x) d x\right],
$$

where the upper and lower signs correspond to symmetric and antisymmetric solutions, respectively.

\section{Example 5.4}

In the previous example we considered the general behavior of $V(x)$ as shown in Fig. 5.10(a). In this example, we use a specific form of $V(x)$ given by [see Fig. 5.11(a)] 


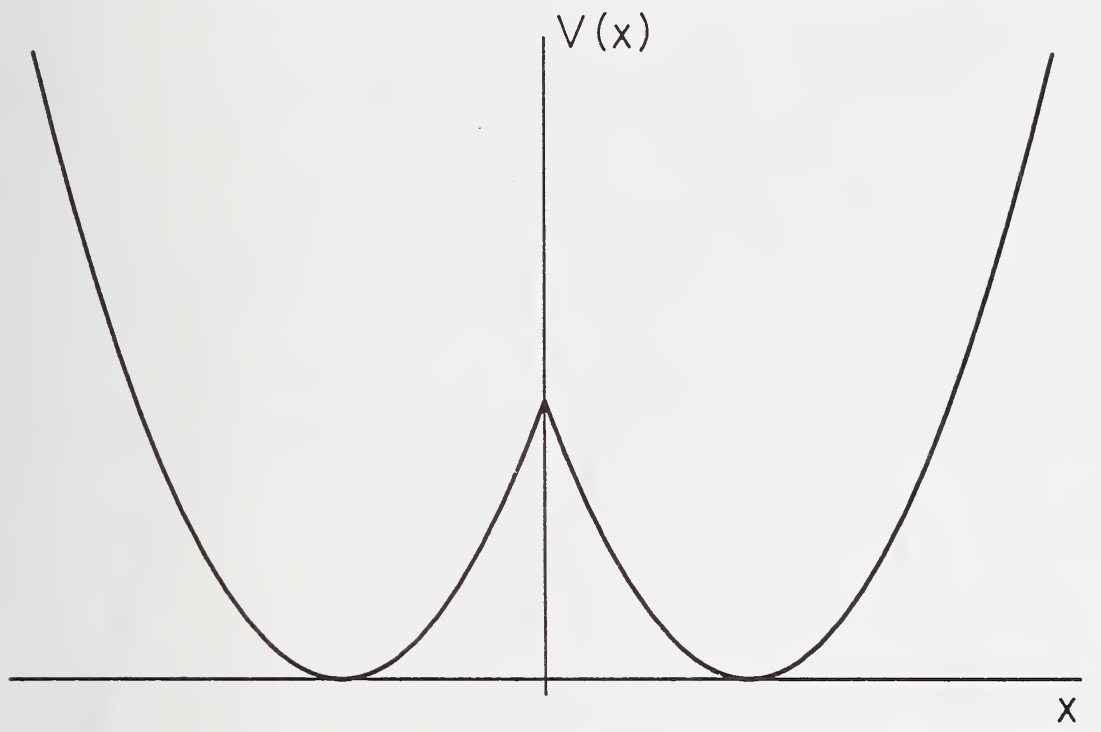

Figure 5.11(a) The potential energy variation for a double oscillator. The corresponding variation of $\Gamma^{2}$ for $E<m \omega^{2} a^{2} / 2$ and $E>m \omega^{2} a^{2} / 2$ are shown in Figs. 5.11(b) and 5.11(c).

$$
V(x)=\frac{1}{2} m \omega^{2}[|x|-a]^{2} .
$$

This potential distribution is usually called the double oscillator. The corresponding variations of $\Gamma^{2}(x)$ are shown in figures 5.11(b) and 5.11(c) for $E<m \omega^{2} a^{2} / 2$ and for $E>m \omega^{2} a^{2} / 2$, respectively. Notice that for $E<m \omega^{2} a^{2} / 2$, there are four turning points and for $E>m \omega^{2} a^{2} / 2$ there are only two turning points. For $E<m \omega^{2} a^{2} / 2$, 


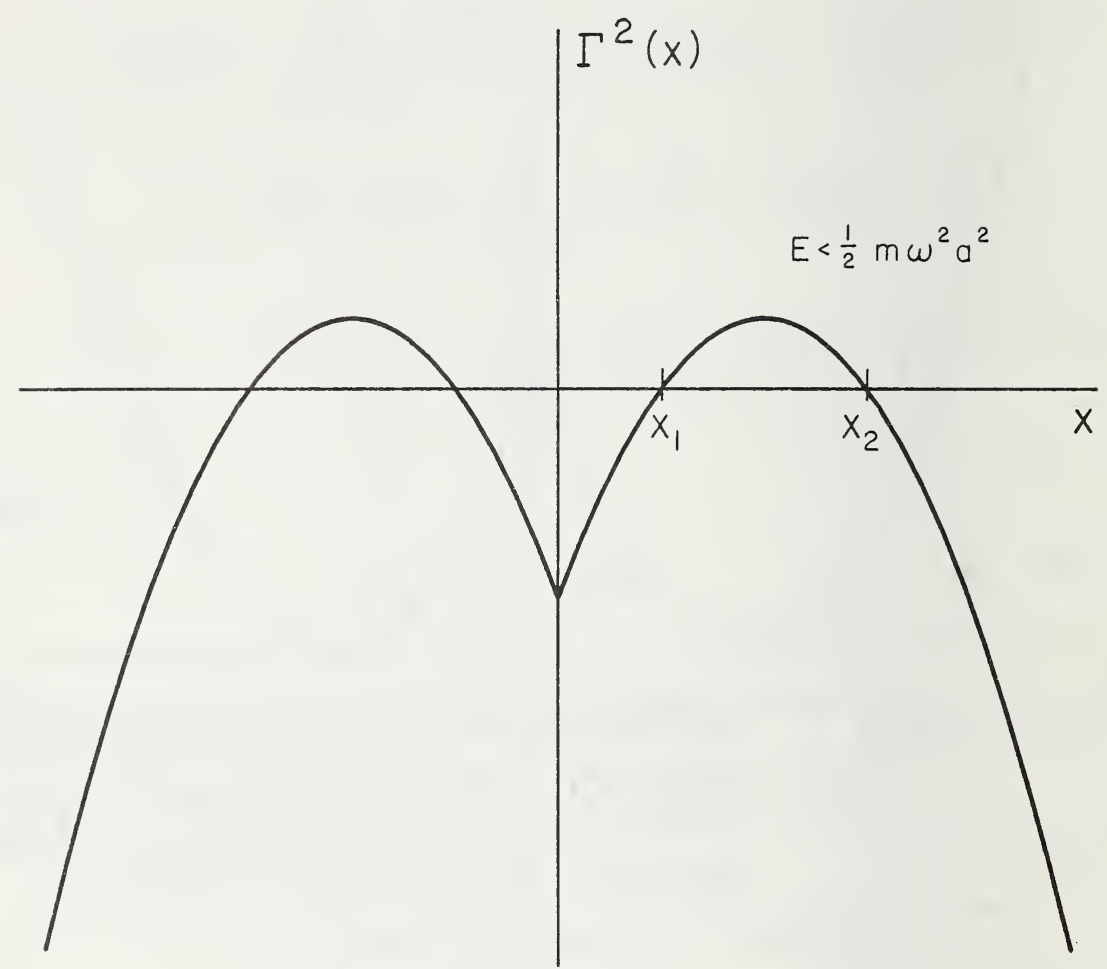

Figure 5.11(b) $\quad \Gamma^{2}(x)$ for $E<m \omega^{2} a^{2} / 2$.

$$
\begin{aligned}
\theta=\int_{x_{1}}^{x_{2}} \Gamma(x) d x & =\sqrt{\frac{2 m}{\hbar^{2}}} \int_{x_{1}}^{x_{2}} \sqrt{E-\frac{m \omega^{2}(x-a)^{2}}{2}} d x \\
& =\int_{-\alpha}^{+\alpha} \sqrt{\alpha^{2}-\xi^{2}} d \xi=\frac{\pi}{2} \alpha^{2},
\end{aligned}
$$

where

$$
\alpha=\sqrt{\frac{2 E}{\hbar \omega}} \quad \text { and } \quad \xi=\sqrt{\frac{m \omega}{\hbar}}(x-a) .
$$




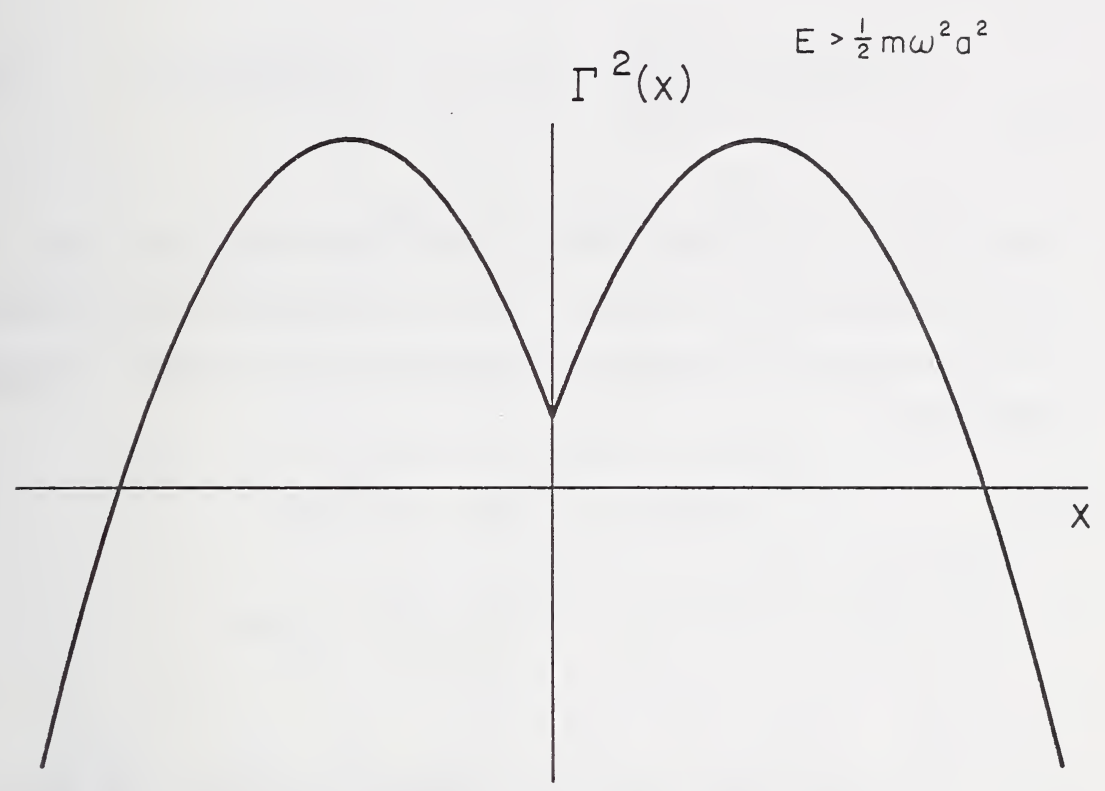

Figure 5.11(c) $\Gamma^{2}(x)$ for $E>m \omega^{2} a^{2} / 2$.

Other integrations can easily be carried out. For $E>m \omega^{2} a^{2} / 2$, we use Eq. (26). The final results are, for $0<\alpha<\alpha_{0}$, $\cot \left(\frac{\pi}{2} \alpha^{2}\right)= \pm \frac{1}{2} \exp \left[-\alpha_{0}\left(\alpha_{0}^{2}-\alpha^{2}\right)^{1 / 2}+\alpha^{2} \cosh ^{-1} \frac{\alpha_{0}}{\alpha}\right]$,

and for $\alpha>\alpha_{0}$,

$$
\frac{\pi}{2} \alpha^{2}+\alpha_{0}\left(\alpha^{2}-\alpha_{0}^{2}\right)^{1 / 2}+\alpha^{2} \sin ^{-1} \frac{\alpha_{0}}{\alpha}=\left(m+\frac{1}{2}\right) \pi,
$$

where 


$$
\alpha=\sqrt{\frac{2 E}{\hbar \omega}} \text { and } \alpha_{0}=\sqrt{\frac{m \omega a^{2}}{\hbar}} \text {. }
$$

Recall that $\alpha$ is the eigenvalue to be determined from Eqs. (61) and (62) and $\alpha_{0}$ is a constant. Before plotting the wavefunctions, it is convenient to introduce the dimensionless variable $\xi$ [see Eq. (60)]; thus,

$$
\begin{aligned}
\int_{x_{2}}^{x} \kappa(x) d x= & \int_{\alpha}^{\xi}\left(\xi^{2}-\alpha^{2}\right)^{1 / 2} d \xi \\
& =\frac{1}{2} \xi\left(\xi^{2}-\alpha^{2}\right)^{1 / 2} \\
& -\frac{1}{2} \alpha^{2} \ln \left|\frac{\xi+\left(\xi^{2}-\alpha^{2}\right)^{1 / 2}}{\alpha}\right| .
\end{aligned}
$$

Hence,

$$
\begin{aligned}
\Psi_{V}(\xi) & =\frac{C}{\left(\xi^{2}-\alpha^{2}\right)^{1 / 4}} \exp \left[-\frac{1}{2} \xi\left(\xi^{2}-\alpha^{2}\right)^{1 / 2}\right. \\
& \left.+\frac{1}{2} \alpha^{2} \ln \left|\frac{\xi+\left(\xi^{2}-\alpha^{2}\right)^{1 / 2}}{\alpha}\right|\right] .
\end{aligned}
$$

Similarly,

$$
\begin{gathered}
\Psi_{I V}(\xi)=\frac{-2 C}{\left(\alpha^{2}-\xi^{2}\right)^{1 / 4}} \sin \left[\frac{\pi}{4}+\frac{\pi}{4} \alpha^{2}-\frac{1}{2} \xi\left(\alpha^{2}-\xi^{2}\right)^{1 / 2}\right. \\
\left.-\frac{1}{2} \alpha^{2} \sin ^{-1} \frac{\xi}{\alpha}\right],
\end{gathered}
$$

and 


$$
\begin{aligned}
\Psi_{I I I}(\xi)= & \frac{C}{\left(\xi^{2}-\alpha^{2}\right)^{1 / 4}}\left[\cos \left(\frac{\pi}{2} \alpha^{2}\right) \exp (\zeta)\right. \\
& \left.+\frac{1}{2} \sin \left(\frac{\pi}{2} \alpha^{2}\right) \exp (-\zeta)\right]
\end{aligned}
$$

where

$$
\begin{aligned}
\zeta(\xi) & =\int_{x}^{x_{1}} \mathrm{~K}(x) d x=\int_{\xi}^{-\alpha}\left(\xi^{2}-\alpha^{2}\right)^{1 / 2} d \xi \\
& =-\frac{1}{2} \xi\left(\xi^{2}-\alpha^{2}\right)^{1 / 2}+\frac{1}{2} \alpha^{2} \ln \left|\frac{\xi+\sqrt{\xi^{2}-\alpha^{2}}}{\alpha}\right| .
\end{aligned}
$$

Figure 5.12 shows the normalized energy eigenvalues $\alpha^{2}$ for the double oscillator as a function of $\alpha_{0}^{2}$; a large value of $\alpha_{0}$ (and hence of $a$ ) implies a large distance between the centers of the two oscillators. The solid and the dashed curves correspond to the exact and the WKB calculations, respectively. As $a \rightarrow \infty, \alpha^{2}$ $(=2 E / \hbar \omega)$ approaches $(2 n+1)$ and the levels become twofold degenerate; thus, the system becomes equivalent to two independent harmonic oscillators. Further, for $a \rightarrow 0$, we have the eigenvalue structure of a single harmonic oscillator. The exact solution for the double oscillator problem is given in terms of the confluent hypergeometric functions. For $V(x)$ given by Eq. (58), the Schrödinger equation is

$$
\frac{d^{2} \psi}{d x^{2}}+\frac{2 m}{\hbar^{2}}\left\{E-\frac{1}{2} m \omega^{2}[|x|-a]^{2}\right\} \Psi=0 .
$$

In terms of the variables 


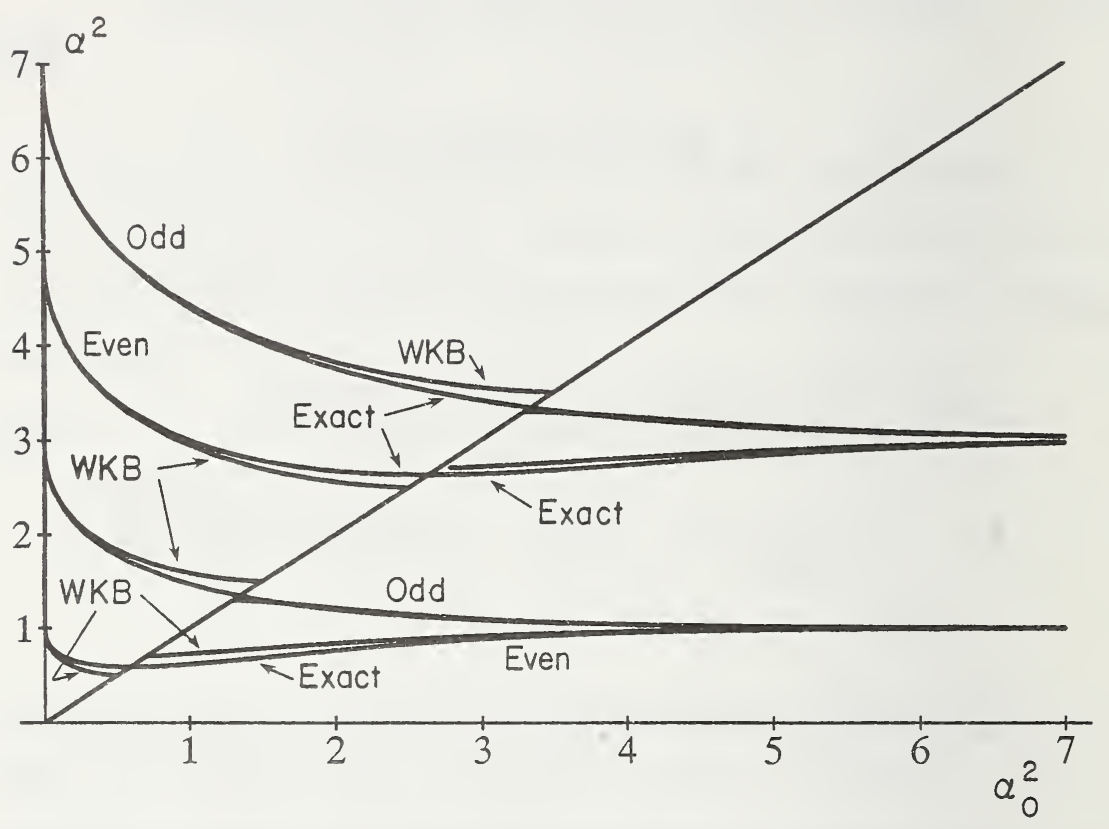

Figure 5.12 Normalized energy eigenvalues for the double oscillator as a function of $\alpha_{0}^{2}$.

$$
\left.\begin{array}{l}
\sigma=\left(\frac{2 m \omega}{\hbar}\right)^{1 / 2}(x-a) \text { for } x>0, \\
\text { and } \\
\tau=\left(\frac{2 m \omega}{\hbar}\right)^{1 / 2}(x+a) \text { for } x<0
\end{array}\right\} \text {, }
$$

Eq. (68) becomes

$$
\frac{d^{2} \psi}{d \sigma^{2}}+\left[\nu+\frac{1}{2}-\frac{1}{4} \sigma^{2}\right] \Psi(\sigma)=0 \quad \text { for } x>0, \text { (70) }
$$




$$
\frac{d^{2} \psi}{d \tau^{2}}+\left[\nu+\frac{1}{2}-\frac{1}{4} \tau^{2}\right] \psi(\tau)=0 \quad \text { for } x<0,
$$

where

$$
v=\frac{1}{2}\left(\frac{2 E}{\hbar \omega}-1\right)=\frac{1}{2}\left(\alpha^{2}-1\right)
$$

The boundary conditions for solving Eqs. (70) and (71) are $\Psi \rightarrow 0$ as $\sigma \rightarrow \infty$ and $\tau \rightarrow-\infty$. For $a=0, \sigma=\tau$ and Eqs. (70) and (71) become equivalent to the linear harmonic oscillator problem and $v$ will then be $0,1,2, \ldots$. The solution of Eq. (70) that vanishes for $\sigma \rightarrow \infty$ is the parabolic cylinder functions $\mathrm{D}_{\nu}(\sigma)$, [Magnus, Oberhettinger, and Soni (1966)] which are related to the confluent hypergeometric function through the relation

$$
\begin{aligned}
D_{v}(\sigma) & =2^{v / 2} \exp \left(-\sigma^{2} / 4\right)\left[\frac{\Gamma\left(\frac{1}{2}\right)}{\Gamma\left(\frac{1-v}{2}\right)}{ }_{1} F_{1}\left(-\frac{v}{2} ; \frac{1}{2} ; \frac{\sigma^{2}}{2}\right)\right. \\
& \left.+\frac{\sigma}{\sqrt{2}} \frac{\Gamma\left(-\frac{1}{2}\right)}{\Gamma\left(-\frac{v}{2}\right)}{ }_{1} F_{1}\left(\frac{1-v}{2} ; \frac{3}{2} ; \frac{\sigma^{2}}{2}\right)\right] .
\end{aligned}
$$

Since $\mathrm{D}_{\nu}(\sigma) \rightarrow 0$ as $\sigma \rightarrow \infty$ we must choose $\mathrm{D}_{\nu}(\sigma)$ for $x>0$ and $\mathrm{D}_{\nu}(-\tau)$ for $\tau<0$. In any case, since $V(-x)=V(x)$, the wavefunctions will be either symmetric or antisymmetric in $x$; thus, we need only the solution fo $x>0$. The eigenvalue equation for the symmetric and antisymmetric wavefunctions are 


$$
\left.D_{v}^{\prime}(\sigma)\right|_{x=0}=0 \quad \text { symmetric }
$$

and

$$
\left.D_{v}(\sigma)\right|_{x=0}=0 \quad \text { antisymmetric . }
$$

The solutions given by Eqs. (74) are plotted in Fig. 5.12. As mentioned earlier, for $a \rightarrow 0$, the solutions correspond to a single oscillator and for $a \rightarrow \infty$ we have twofold degeneracy corresponding to two noninteracting oscillators.

\section{Example 5.5}

In this and the following examples we illustrate the use of a convenient procedure that allows the solution to the WKB quantization condition [see Eq. (26)] in special cases. We consider the following potential energy distribution (see Fig. 5.13)

$$
V(x)=-V_{0} \operatorname{sech}^{2}\left(\frac{x}{a}\right) .
$$

Thus,

$$
\Gamma^{2}(x)=\frac{2 m V_{0}}{\hbar^{2}}\left[\frac{E}{V_{0}}+\operatorname{sech}^{2}\left(\frac{x}{a}\right)\right],
$$

and the quantization condition [Eq. (26)] becomes

$$
\left(\frac{2 m V_{0} a^{2}}{\hbar^{2}}\right)^{1 / 2} \int_{-\alpha(\xi)}^{+\alpha(\xi)}\left[\operatorname{sech}^{2} \xi-\xi\right]^{1 / 2} d \xi=\left(n+\frac{1}{2}\right) \pi,
$$

where 


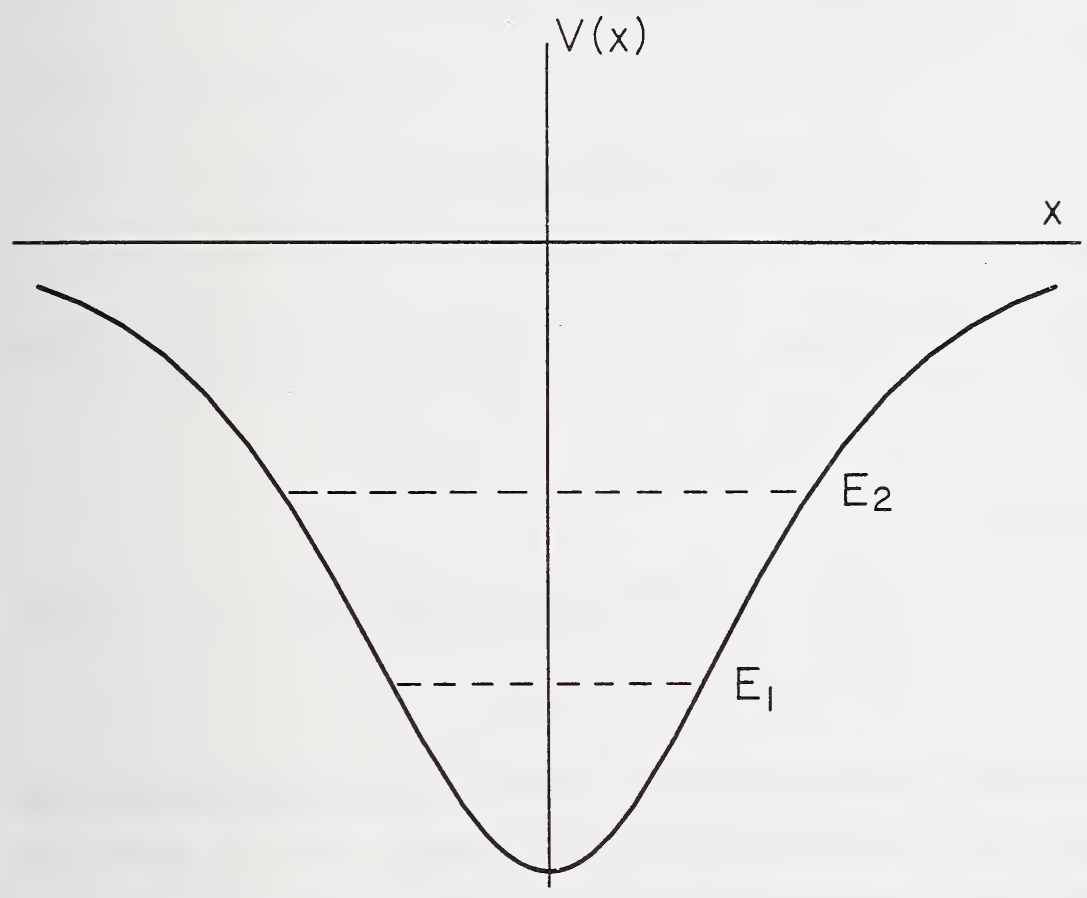

Figure 5.13 Potential energy distribution for Fig. 5.5.

$$
\xi=\frac{x}{a}, \quad \mathscr{E}=-\frac{E}{V_{0}} \text { and } \alpha=\operatorname{sech}^{-1} \sqrt{\mathscr{E}}
$$

For bound states $E<0$ and, since $E$ can never be less than the minimum value of $V$, we have

$$
0<\mathscr{b}<1 \text {. }
$$

Equation (77) requires the solution of the integral 


$$
J(\mathscr{E})=\int_{-\alpha(\xi)}^{+\alpha(\mathscr{\xi})}\left[\operatorname{sech}^{2} \xi-\mathscr{E}\right]^{1 / 2} d \xi
$$

If

then

$$
J(x)=\int_{g(x)}^{f(x)} F(x, y) d y,
$$

$$
\frac{d J}{d x}=\int_{g(x)}^{f(x)} \frac{\partial F}{\partial x} d y+F[x, f(x)] \frac{d f}{d x}-F[x, g(x)] \frac{d g}{d x} .
$$

Therefore

$$
\frac{d J}{d \mathscr{E}}=-\frac{1}{2} \int_{-\alpha}^{+\alpha}\left[\operatorname{sech}^{2} \xi-\mathscr{E}\right]^{1 / 2} d \xi
$$

In our case, the last two terms vanish because the limits are the turning points where the integrand vanishes. In fact, since this always happens, this technique is very often used in the evaluation of the integral appearing in the WKB quantization condition. If we now make the transformation

$$
\eta=\sinh \xi,
$$

then 


$$
d \eta=\cosh \xi d \xi \Rightarrow d \xi=\frac{d \eta}{\left(1+\eta^{2}\right)^{1 / 2}},
$$

and Eq. (83) becomes

$$
\begin{aligned}
\frac{d J}{d \mathscr{E}} & =-\frac{1}{2} \int \frac{1}{\left(\frac{1}{1+\eta^{2}}-\mathscr{E}\right)^{1 / 2}} \frac{d \eta}{\left(1+\eta^{2}\right)} \\
& =-\frac{1}{2 \sqrt{\mathscr{E}}}\left[\sin ^{-1}\left(\sqrt{\frac{\mathscr{E}}{1-\mathscr{E}}} \sinh \xi\right)\right]_{-\alpha}^{+\alpha} \\
& =-\frac{\pi}{2 \sqrt{\mathscr{E}}} .
\end{aligned}
$$

Simple integration gives

$$
J(\mathscr{E})=\pi(1-\sqrt{\mathscr{E}})
$$

where we have used the condition $J(1)=0$ [see Eq. (80]). Thus, the energy eigenvalues are given by

$$
E_{n}=\frac{-\hbar^{2}}{2 m a^{2}}\left[\left(\frac{2 m V_{0} a^{2}}{\hbar^{2}}\right)^{1 / 2}-\left(n+\frac{1}{2}\right)\right]^{2} \text {. }
$$

In this case we can also obtain an exact solution of the Schrödinger equation; the final result is

$$
E_{n}=-\frac{\hbar^{2}}{2 m a^{2}}\left[\frac{v}{2}-\left(n+\frac{1}{2}\right)\right]^{2},
$$


where

$$
v=\left(\frac{8 m V_{0} a^{2}}{\hbar^{2}}+1\right)^{1 / 2}
$$

The WKB eigenvalues are recovered if the first term in the brackets is large with respect to 1 . The eigenfunctions are

$$
\psi(\sigma)=[1-\sigma]^{-\frac{v-1}{2}} W(\sigma),
$$

where $\sigma=-\sinh ^{2}(x / a)$ and $W(\sigma)$ is the polynomial solution of the hypergeometric equation.

Problem 5.2. Obtain the WKB eigenfunctions of Example 5.5 and compare them with the exact solutions.

\subsection{The WKB Method for Three-Dimensional Problems}

For a spherically symmetric potential energy distribution $V(r)$, the radial part of the Schrödinger equation is given by [Schiff (1968)]

$$
\frac{d^{2} R}{d r^{2}}+\frac{2}{r} \frac{d R}{d r}+\frac{2 m}{\hbar^{2}}\left[E-V(r)-\frac{l(l+1) \hbar^{2}}{2 m r^{2}}\right] R(r)=0
$$

where $\ell(\ell+1) \hbar^{2}$ (with $\ell=0,1,2, \ldots$ ) represents the eigenvalues of the square of the angular momentum operator $L^{2}$ and $m$ is the mass of the particle. If we make the transformation

$$
R(r)=\frac{u(r)}{r},
$$


then Eq. (89) becomes

$$
\frac{d^{2} u}{d r^{2}}+\frac{2 m}{\hbar^{2}}\left[E-V(r)-\frac{\ell(\ell+1) \hbar^{2}}{2 m r^{2}}\right] u(r)=0 .
$$

Although Eq. (91) has the same form as Eq. (1), the independent variable $r$ goes from 0 to $\infty$. To use the asymptotic forms, we introduce a new variable $x$ such that $r=0$ corresponds to $x=$ $\infty$; this is achieved through the transformation

$$
r=\exp (x) \text {. }
$$

In terms of the variable $x$, Eq. (91) becomes

$$
\begin{aligned}
& \frac{d^{2} u}{d x^{2}}-\frac{d u}{d x}+\exp (2 x) \frac{2 m}{\hbar^{2}}[E-V \\
& \left.-\frac{\ell(\ell+1) \hbar^{2}}{2 m} \exp (-2 x)\right] u(x)=0 .
\end{aligned}
$$

To remove the first derivative [so it is in the form of Eq. (1)], we introduce the transformation

$$
\chi(x)=u(x) \exp \left(-\frac{x}{2}\right)
$$

The function $\chi(\mathrm{x})$ satisfies the equation

$$
\frac{d^{2} \chi}{d x^{2}}+\Gamma^{2}(x) \chi(x)=0
$$

which is of the desired form, where 


$$
\begin{aligned}
\Gamma^{2}(x) & =\exp (2 x) \frac{2 m}{\hbar^{2}}\left[E-V\left(e^{x}\right)\right. \\
& \left.-\frac{\left(\ell+\frac{1}{2}\right)^{2} \hbar^{2}}{2 m} \exp (-2 x)\right] .
\end{aligned}
$$

In terms of the original variable $r$,

$$
\int^{x} \Gamma(x) d x=\int^{r} \Gamma_{1}(r) d r,
$$

where

$$
\Gamma_{1}^{2}(r)=\frac{2 m}{\hbar^{2}}\left[E-V(r)-\frac{\left(\ell+\frac{1}{2}\right)^{2} \hbar^{2}}{2 m r^{2}}\right] .
$$

To find the bound state energies, we must solve the equation

$$
\int_{r_{1}}^{r_{2}} \Gamma_{1}(r) d r=\left(n+\frac{1}{2}\right) \pi,
$$

where $r_{1}$ and $r_{2}$ are the turning points, where $\Gamma_{1}(\mathrm{r})$ vanishes. Equation (99) shows that the WKB solution to the radial part of the Schrödinger equation is equivalent to Eq. (1) when $\ell(\ell+1)$ is replaced by $(\ell+1 / 2)^{2}$.

\section{Example 5.6}

We consider the case of the hydrogen atom, for which

$$
V(r)=-Z \frac{e^{2}}{r} .
$$


The WKB quantization condition is given by

$\sqrt{\frac{2 m}{\hbar^{2}}} \int_{r_{1}}^{r_{2}} \sqrt{E+\frac{Z e^{2}}{r}-\frac{\left(\ell+\frac{1}{2}\right)^{2} \hbar^{2}}{2 m r^{2}}} d r=\left(n+\frac{1}{2}\right) \pi$,

or

$$
\sqrt{2} J(\mathscr{E})=\left(n+\frac{1}{2}\right) \pi
$$

where

$$
J(\mathscr{G})=\int_{\xi_{1}}^{\xi_{2}}\left[-\mathscr{G}-V_{e f f}(\xi)\right]^{1 / 2} d \xi,
$$

with

$$
\begin{gathered}
V_{\text {eff }}(\xi)=-\frac{Z}{\xi}+\frac{\alpha}{\xi^{2}}, \\
\mathscr{E}=-\frac{E}{\left(\frac{m e^{4}}{\hbar^{2}}\right)}, \\
\xi=\frac{r}{\left(\frac{\hbar^{2}}{m e^{2}}\right)} \\
\alpha=\frac{1}{2}\left(l+\frac{1}{2}\right)^{2} .
\end{gathered}
$$


Following the method used in Example 5.5, we obtain

$$
\frac{d J}{d \mathscr{E}}=-\frac{\pi Z}{4 \mathscr{E}^{3 / 2}}
$$

Further, the minimum value of $V_{\text {eff }}(\xi)$ is $-Z^{2} / 4 \alpha$, which occurs at $\xi=2 \alpha / Z$. Thus,

$$
J\left(\mathscr{E}=-\frac{Z^{2}}{4 \alpha}\right)=0,
$$

because $\xi_{1}$ becomes equal to $\xi_{2}$. Equation (105) can now be integrated. Using Eq. (106), we obtain

$$
\mathscr{b}=\frac{Z^{2}}{2(n+\ell+1)^{2}},
$$

which is also the exact result with total quantum number $(n+\ell+1)$. 


\section{MAF SOLUTIONS TO EIGENVALUE PROBLEMS}

\subsection{Introduction}

In this chapter we reconsider the eigenvalue problem, but we use the Modified Airy Function (MAF) solutions that were introduced in Chapter 4. As in previous chapters, we will consider examples that yield to exact solutions, to demonstrate the utility and strength of the method. The formulation is based on papers by Goyal, Gallawa, and Ghatak (1991a,b) and Ghatak, Gallawa, and Goyal (1991).

The equation of interest is again

$$
\frac{d^{2} \psi}{d x^{2}}+\Gamma^{2}(x) \psi(x)=0,
$$

where $\Gamma^{2}(x)$ includes the (unknown) eigenvalue. If the equation represents an integrated optical waveguide problem, $\Gamma^{2}$ will include the propagation constant for the mode in question as the unknown eigenvalue. For quantum mechanical problems, the unknown eigenvalue will be the characteristic (allowed) energy levels. The equations are identical but the parameters represent different physical quantities. 


\subsection{Formulation of the Problem}

The eigenvalue problems follow the format already established. To be definite, we will formulate our approach around a planar waveguide problem, wherein we consider a planar optical waveguide having a nonuniform refractive index profile in the core of the waveguide. As before, we assume and suppress exp[i( $\omega t$ $\beta z)]$ variation with time and $z$. Propagation is thus assumed to be in the $z$ direction and, because of this exponential variation with $z$, we explicitly assume that the waveguide is uniform in $z$. The core of the waveguide is the region $|x| \leq a$. The problems of interest have refractive index, $n=n(x)$ in the core and $n=$ constant outside the core (in the cladding) of the guide. $n(x)$ may be quite general but we will restrict attention to those variations that lend themselves to exact solutions of the wave equation. The relationship between the modal propagation constant, $\beta$, and $\Gamma$ is

$$
\Gamma^{2}(x)=k_{0}^{2} n^{2}(x)-\beta^{2},
$$

where $k_{0}=2 \pi / \lambda_{0}$ and $\lambda_{0}$ is the free-space wavelength. For future reference we define here the effective refractive index $n_{\mathrm{e}}$ for the mode in question:

$$
n_{e}=\frac{\beta}{k_{0}}
$$

The sign of $\Gamma^{2}(x)$ depends on whether $n(x)$ is greater than or less than $n_{\mathrm{e}}$. The solutions of eq(1) are growing or decaying exponential functions when $\Gamma^{2}$ is negative and the solutions are oscillatory when the $\Gamma^{2}$ is positive. The turning point is the point 
(on the $x$ axis) at which $\Gamma^{2}$ changes sign.

The form of the oscillatory functions depend on $n(x)$. If $n(x)$ is constant, trigonometric functions are encountered. For $n(x)$ that is nonconstant and not amenable to a closed form solution, we again propose the Modified Airy Function (MAF) solutions.

Following the development of Chapter 4, we take the MAF solution to Eq. (1) as

$$
\psi(x)=C_{1} \frac{A i[\xi(x)]}{\sqrt{\xi^{\prime}(x)}}+C_{2} \frac{B i[\xi(x)]}{\sqrt{\xi^{\prime}(x)}} .
$$

As before,

$$
\xi(x)=\left[\int_{x_{t}}^{x} \frac{3}{2} \sqrt{-\Gamma^{2}(x)} d x\right]^{2 / 3},
$$

where $x_{\mathrm{t}}$ is the turning point. Choosing the lower limit of integration to be the turning point causes the solution to become exact if $\Gamma^{2}$ happens to be linear in $x$.

As seen previously,

$$
\frac{1}{\sqrt{\xi^{\prime}(x)}}=\frac{|\xi|^{\frac{1}{4}}}{\left|\Gamma^{2}(x)\right|^{\frac{1}{4}}} .
$$

Equation (4) is identical to the WKB solution if we use the 
asymptotic expansion of Airy functions together with Eqs. (6) and (5). That is, this solution and the WKB solution are identical in the region far from the turning point. The eigenvalue equations are obtained when the boundary conditions are applied.

\subsection{Examples}

\section{Example 6.1}

Consider first the profile shown in Fig. 6.1, for which an exact solution is known. The square of the refractive index decreases exponentially for $x>0$ and is constant for $x<0$. Mathematically,

$$
\begin{gathered}
n^{2}(x)=n_{2}^{2}+\left(n_{1}^{2}-n_{2}^{2}\right) \exp \left(-\frac{x}{d}\right) \quad \text { for } x>0, \\
n^{2}(x)=n_{c}^{2} \quad \text { for } x<0,
\end{gathered}
$$

where $n_{1}, n_{2}$, and $n_{\mathrm{c}}$ are the refractive index values shown symbolically in Fig. 6.1 and $d$ is the diffusion depth of the waveguide. It will be convenient later to use the normalized frequency $V$ defined as

$$
V=\frac{2 \pi}{\lambda_{0}} d \sqrt{n_{1}^{2}-n_{2}^{2}} .
$$

$\lambda_{0}$ is the operating free-space wavelength. This profile was chosen because it has an exact solution, making it useful to demonstrate the accuracy of the MAF solutions. 


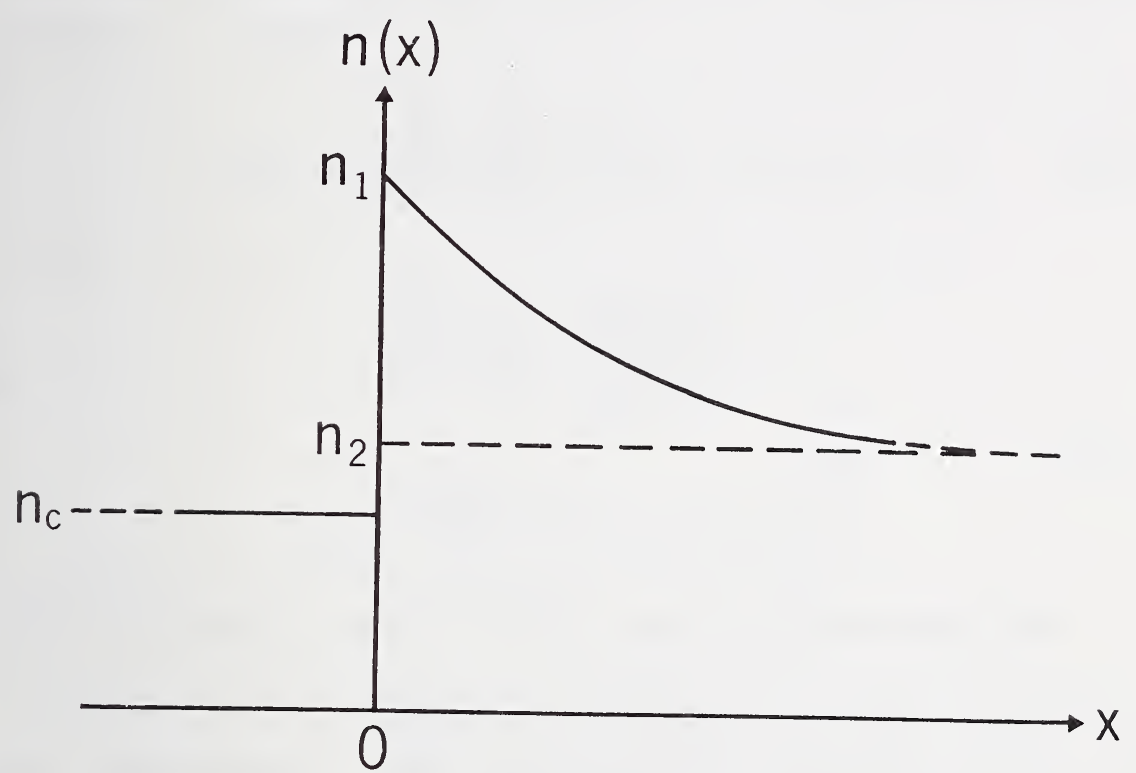

Figure 6.1 Refractive index profile for the waveguide of Example 6.1.

We rewrite the governing equation in terms of normalized (dimensionless) quantities using a convenient change of variables. For the profile given by Eq. (7), we get

$$
\begin{gathered}
\frac{d^{2} \psi}{d} X^{2}+V^{2}(\exp (-X)-b) \psi=0 \quad \text { for } X>0, \\
\frac{d^{2} \psi}{d X^{2}}-V^{2}(b+B) \psi=0 \quad \text { for } X<0,
\end{gathered}
$$

where we have introduced additional dimensionless variables, 


$$
\begin{gathered}
X=x / d, \\
B=\frac{n_{2}^{2}-n_{c}^{2}}{n_{1}^{2}-n_{2}^{2}} . \\
b=\frac{n_{e}^{2}-n_{2}^{2}}{n_{1}^{2}-n_{2}^{2}}, \\
n_{e}=\beta / k_{0} .
\end{gathered}
$$

The exact solution of Eqs. (9) and (10) are [Conwell (1973)]:

$$
\begin{array}{ll}
\Psi(X)=\frac{J_{v}\left[2 V \exp \left(-\frac{X}{2}\right)\right]}{J_{v}(2 V)} & \text { for } X>0, \\
\Psi(X)=\exp (X V \sqrt{b+B}) & \text { for } X<0,
\end{array}
$$

where

$$
v=2 V \sqrt{b} \text {. }
$$

Equation (13) satisfies the boundary condition, $\Psi(X) \rightarrow 0$ as $X \rightarrow \pm$ $\infty$ and the multiplying constants have been chosen so that $\Psi(0)=1$. Requiring the continuity of $\Psi^{\prime}(X)$ at $X=0$ yields the eigenvalue equation for the normalized propagation constant $b$ :

$$
\frac{J_{v}^{\prime}(2 V)}{J_{v}(2 V)}=-\left(\frac{n_{e}^{2}-n_{c}^{2}}{n_{1}^{2}-n_{2}^{2}}\right)^{1 / 2}
$$


The $M A F$ solutions are now written directly, using the results of Chapter 4. We take

$$
\psi(X)=F(X) A i[\xi(X)], \text { for } X>0
$$

where

$$
\begin{gathered}
F(X)=\frac{\text { constant }}{\sqrt{\xi^{\prime}(X)}}, \\
\xi(X)=\left[\frac{3}{2} \int_{X_{t}}^{X}\left[-\Gamma^{2}(X)\right]^{1 / 2} d X\right]^{2 / 3},
\end{gathered}
$$

and $X=X_{\mathrm{t}}$ is the turning point. For $X<0$, the solution given by Eq. (13) holds. We have neglected the solution that is proportional to $B i(\xi)$ as it diverges for large $X$. We can now write the solution that gives $\Psi(0)=1$

$$
\Psi(X)=\left(\frac{\xi_{0}^{\prime}}{\xi^{\prime}}\right)^{1 / 2} \frac{A i(\xi)}{A i\left(\xi_{0}\right)} \quad \text { for } X>0,
$$

and $\xi(X)$ can be written in closed form, as 


$$
\begin{aligned}
& \xi(X)=-[3 V(\sqrt{\exp (-X)-b} \\
& \left.\left.-\sqrt{b} \tan ^{-1} \sqrt{\frac{\exp (-X)}{b}-1}\right)\right]^{2 / 3} \\
& \text { for } X<X_{t}, \\
& \xi(X)=[3 V(-\sqrt{b-\exp (-X)} \\
& \left.\left.+\frac{\sqrt{b}}{2} \ln \frac{\sqrt{b}+\sqrt{b-\exp (-X)}}{\sqrt{b}-\sqrt{b-\exp (-X)}}\right)\right]^{2 / 3} \\
& \text { for } X>X_{t} .
\end{aligned}
$$

The turning point is located at $X_{\mathrm{t}}=-\ln (b)$. The subscript 0 indicates the value of the function at $X=0$. The eigenvalue equation can now be written by invoking the continuity of $\Psi^{\prime}(X)$ at $X=0$ :

$$
\frac{V(B+b)^{1 / 2}}{\xi_{0}^{\prime}}=\frac{A i^{\prime}\left(\xi_{0}\right)}{A i\left(\xi_{0}\right)}-\frac{1}{2} \frac{\xi_{0}^{\prime \prime}}{\left(\xi_{0}^{\prime}\right)^{2}}
$$


To illustrate the method, we used $m_{2}=2.177, n_{\mathrm{c}}=1$, and $n_{1}^{2}-n_{2}^{2}=0.187$ in our calculations. The value of $d$ was varied to obtain results as a function of $V$. The calculated values of $b$ are given in Table 6.1 together with the exact values [obtained by solving Eq. (15)] and those calculated by the WKB method. The agreement is very good compared to $W K B$ for all values of $V$. Even at $V=1.5$ (very near to cutoff), the error is only 3 percent. The corresponding error of the WKB method is 8 percent.

\begin{tabular}{|c|c|c|c|}
\hline \multicolumn{4}{|c|}{$\begin{array}{l}\text { Table } 6.1 . \\
\text { Normalized propagation constants. }\end{array}$} \\
\hline V & Exact & MAF & WKB \\
\hline 1.5 & 0.035007 & 0.036088 & 0.037833 \\
\hline 2.0 & 0.104954 & 0.105896 & 0.108613 \\
\hline 2.5 & 0.171442 & 0.172159 & 0.175311 \\
\hline 3.0 & 0.229188 & 0.229736 & 0.233076 \\
\hline 3.5 & 0.278650 & 0.279081 & 0.282486 \\
\hline 4.0 & 0.321179 & 0.321520 & 0.324927 \\
\hline 5.0 & 0.390292 & 0.390522 & 0.393845 \\
\hline 6.0 & 0.444075 & 0.444241 & 0.447436 \\
\hline 7.0 & 0.487244 & 0.487369 & 0.490429 \\
\hline 8.0 & 0.522776 & 0.522874 & 0.525803 \\
\hline
\end{tabular}


We call attention to the fact that the error $\Delta b$ in $b$ is related to error $\Delta \beta$ in $\beta$ by the relation [see Eq. (12)],

$$
\frac{\Delta b}{b} \approx \frac{\Delta \beta}{\beta} \frac{n_{1}}{n_{1}-n_{2}} \frac{1}{b} \text {. }
$$

Thus, an error of 3 percent in $b$ in this case will correspond to an error of about 0.002 percent in $\beta$.

Figure 6.2 shows $\Psi$ versus $X$ for $V=4$ as calculated by Eq. (18) (MAF method) and Eq. (13) (exact). The figure shows no discernable difference between the two curves, even at the turning point.

In Fig. 6.3 we give a plot of $\left(\Psi_{\mathrm{MAF}}-\Psi_{\mathrm{EXACT}}\right)$. This figure illustrates the strength of the MAF method since the results are quite good even at the turning points. In Fig. 6.4 we give the fractional error in $b$ as a function of $V$.

The profile of the foregoing example will be encountered again in Chapter 7 to illustrate how the perturbation method used in conjunction with the MAF solution can improve (quite dramatically) the accuracy of the calculated eigenvalues.

\section{Example 6.2}

For this example we consider a symmetric profile for which 


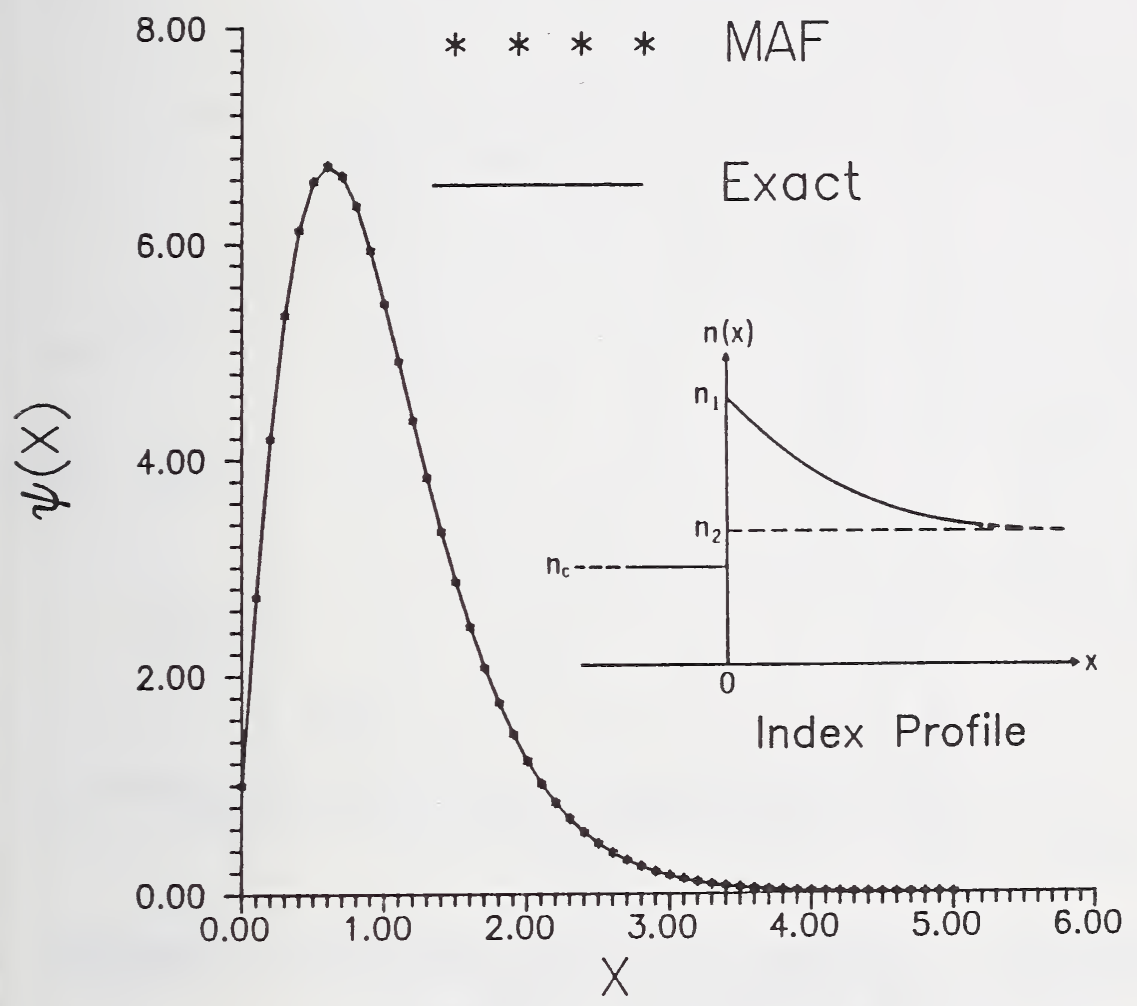

Figure 6.2 Wavefunctions for the waveguide of Example 6.1, $V=4$.

$$
\Gamma^{2}(-x)=\Gamma^{2}(x)
$$

For a bound waveguide mode, the wave function must approach 0 as $x$ approaches $\pm \infty$, so we again reject the solution that is proportional to the $B i$ function. Hence, we take 


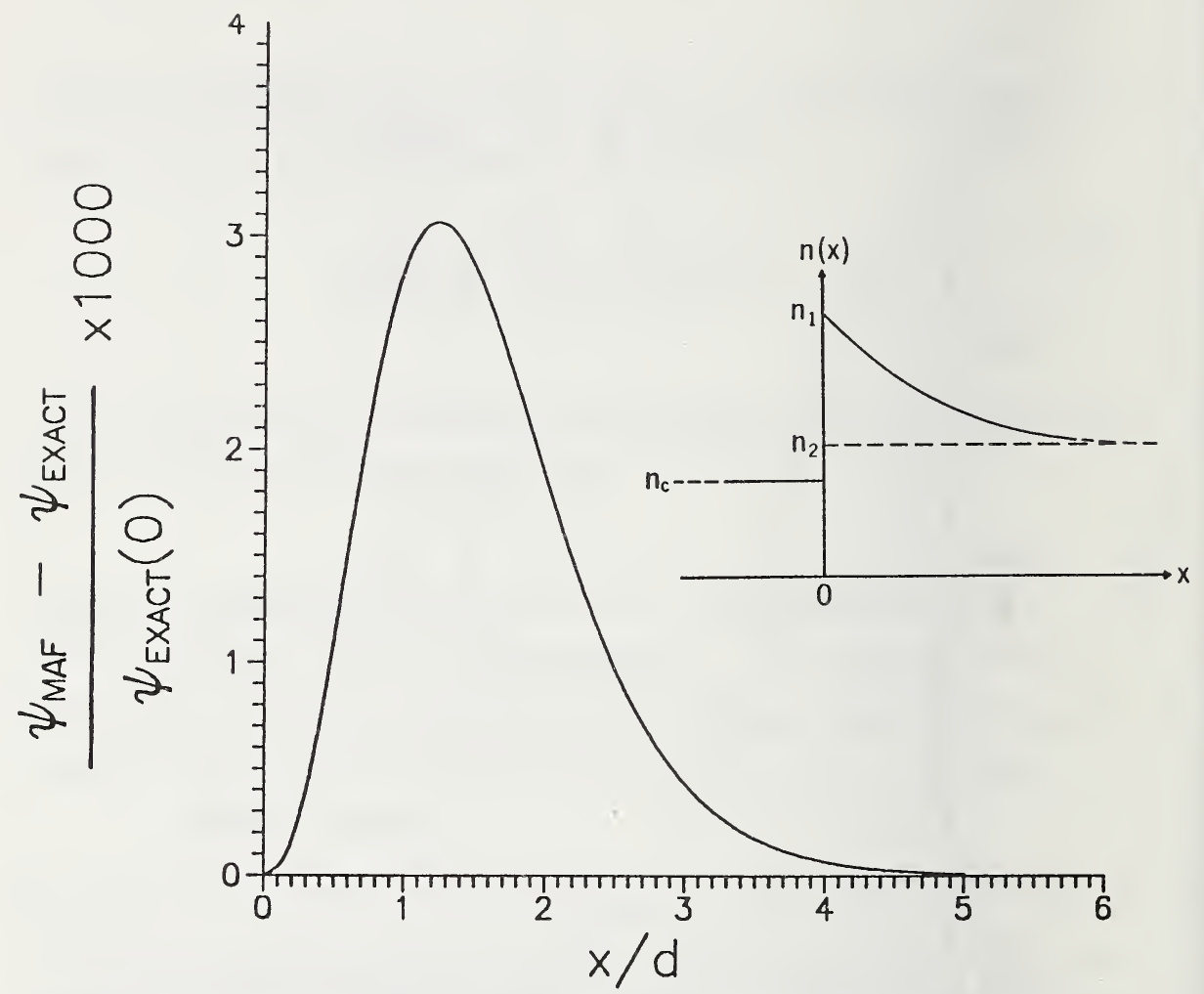

Figure 6.3 Difference between the exact and the $M A F$ solutions for Example 6.1, $V=4$.

$$
\Psi(x)=\frac{C_{1}}{\sqrt{\xi^{\prime}(x)}} \operatorname{Ai}[\xi(x)], \quad x>0 .
$$

Equation (22) suggests that the solutions are either symmetric or antisymmetric in $x$, so

$$
\Psi^{\prime}(0)=0 \quad \text { (symmetric solution), }
$$

or 


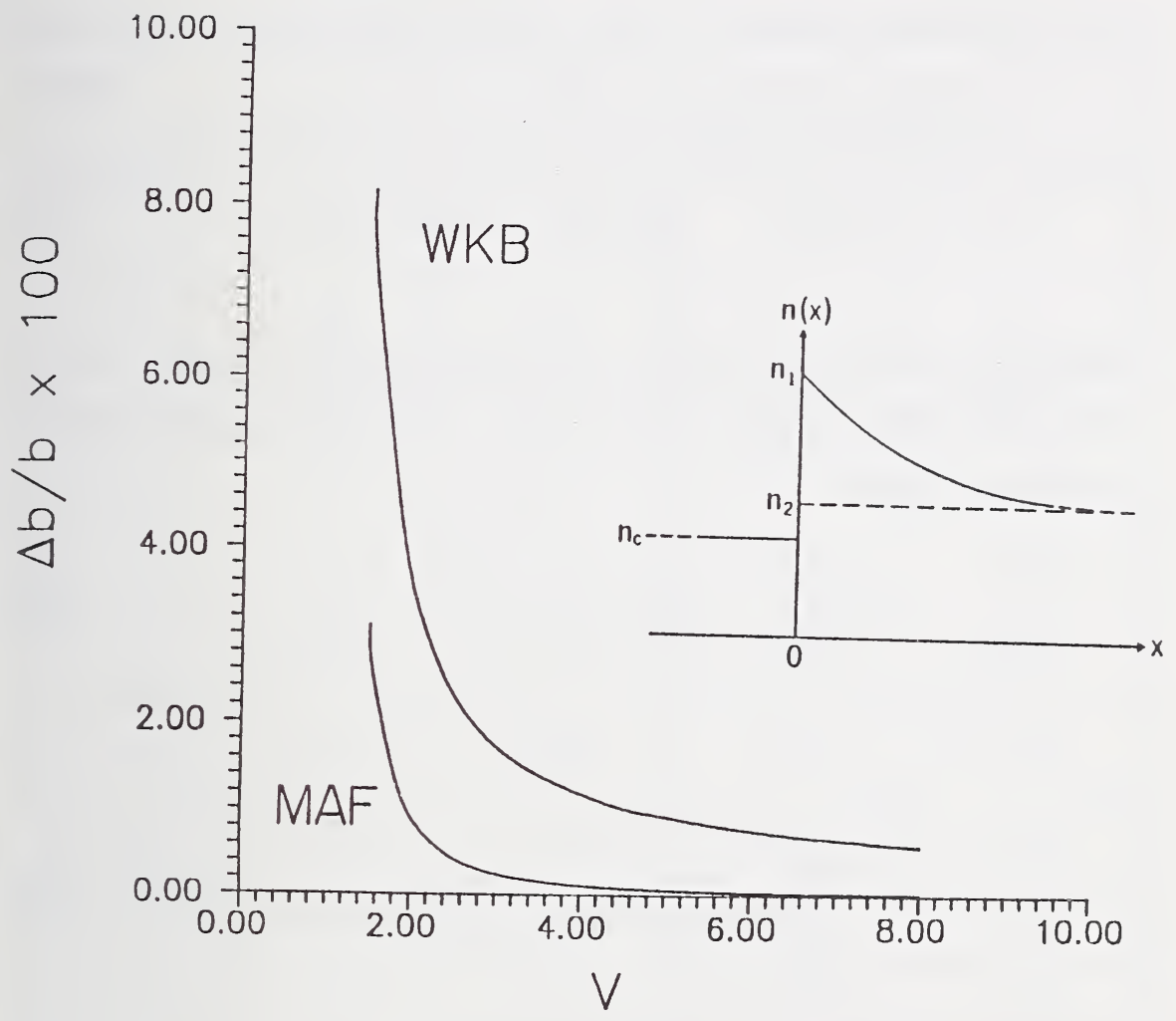

Figure 6.4. Fractional error in normalized propagation constant for Example 6.1, as a function of $V$.

$\psi(0)=0$ (antisymmetric solution) .

Invoking these conditions leads to the eigenvalue equations $\frac{A i^{\prime}(\xi(0))}{A i(\xi(0))}=\frac{1}{2} \frac{\xi^{\prime \prime}(0)}{\left[\xi^{\prime}(0)\right]^{2}} \quad$ (symmetric solution),

and

$$
A i[\xi(0)]=0 \quad \text { (antisymmetric solution) . }
$$


The eigenvalue equation for the antisymmetric solution [Eq. (24)] can be put in a simple form. Let $-Z_{\text {an }}(n=1,2,3, \ldots)$ denote the nth zero of the $A i$ function; i.e.,

$$
\operatorname{Ai}\left(Z_{a n}\right)=0 \text {, }
$$

where the subscript $a$ refers to the antisymmetric solution. Using Eqs. (5), (24), and (25) and assuming $\Gamma^{2}(x)$ to be positive from 0 to $X_{t}$, we obtain

$$
\xi(0)=-\left[\frac{3}{2} \int_{0}^{x_{t}} \Gamma(x) d x\right]^{2 / 3}=-Z_{a n},
$$

or

$$
\int_{0}^{x_{t}} \Gamma(x) d x=\left(\zeta_{a n}+1 / 2\right) \pi / 2,
$$

where

$$
\zeta_{a n}=\left(\frac{4}{3 \pi} Z_{a n}^{3 / 2}-1 / 2\right)
$$

Table 6.2 gives the values of $Z_{\text {an }}$ [as obtained from Abramowitz and Stegun (1970)] and the corresponding values of $\zeta_{\mathrm{an}}$, which are universal constants. Equation (27) is similar to the WKB quantization condition except that (for the $W K B$ case) $\zeta_{\text {an }}$ is replaced by odd integers. 
Table 6.2.

Zeros appropriate to MAF quantization condition.

\begin{tabular}{|c|c|c||c|c||}
\hline \hline & \multicolumn{2}{|c||}{ Antisymmetric modes } & \multicolumn{2}{c||}{ Symmetric modes } \\
\hline $\mathrm{n}$ & $\mathrm{Z}_{\text {an }}$ & $\zeta_{\text {an }}$ & $\mathrm{Z}_{\text {sn }}$ & $\zeta_{\text {sn }}$ \\
\hline 1 & 2.33811 & 1.01734 & 1.20348 & 0.06033 \\
\hline 2 & 4.08795 & 3.00790 & 3.27162 & 2.01150 \\
\hline 3 & 5.52056 & 5.00508 & 4.83082 & 4.00630 \\
\hline 4 & 6.78671 & 7.00374 & 6.16988 & 6.00435 \\
\hline 5 & 7.94413 & 9.00295 & 7.37677 & 8.00331 \\
\hline 6 & 9.02265 & 11.00244 & 8.49195 & 10.0027 \\
\hline 7 & 10.04017 & 13.00208 & 9.53820 & 12.0023 \\
\hline 8 & 11.00852 & 15.00181 & 10.52992 & 14.0020 \\
\hline 9 & 11.93602 & 17.00161 & 11.47696 & 16.0017 \\
\hline 10 & 12.82878 & 19.00144 & 12.38642 & 18.0015 \\
\hline
\end{tabular}

Similarly, we may consider the symmetric solution [Eq. (23)]. From Eq. (5) of Chapter 4,

$$
\xi\left(\xi^{\prime}\right)^{2}=-\Gamma^{2}(x) .
$$

Differentiation with respect to $x$ yields 


$$
\left(\xi^{\prime}\right)^{3}+2 \xi \xi^{\prime} \xi^{\prime \prime}=-\frac{d}{d x}\left(\Gamma^{2}(x)\right) .
$$

The right side of this equation will vanish at $x=0$ for a symmetric profile [see Eq. (22)]. Hence, at $x=0$, Eq. (30) becomes

$$
\frac{1}{2} \frac{\xi^{\prime \prime}(0)}{\xi^{\prime}(0)^{2}}=-\frac{1}{4 \xi(0)}
$$

Equation (23) therefore becomes

$$
\xi(0) A i^{\prime}[\xi(0)]+\frac{1}{4} A i[\xi(0)]=0 .
$$

Consider the function $\chi A i^{\prime}(\chi)+A i(\chi) / 4$ (which is plotted in Fig. 6.5). Let $-Z_{\mathrm{sn}}$ be the $n$th zero of this function $(\mathrm{n}=1,2,3$, ...; that is

$$
-Z_{s n} A i^{\prime}\left(-Z_{s n}\right)+\frac{1}{4} A i\left(-Z_{s n}\right)=0
$$

where the subscript $s$ refers to the symmetric solutions. Using Eqs. (5), (23), and (33), and assuming $\Gamma^{2}(x)$ to be positive from 0 to $x_{t}$, we obtain [see Eq. (26)]

$$
\xi(0)=-\left[\frac{3}{2} \int_{0}^{x_{t}} \Gamma(x) d x\right]^{2 / 3}=-Z_{s n}
$$




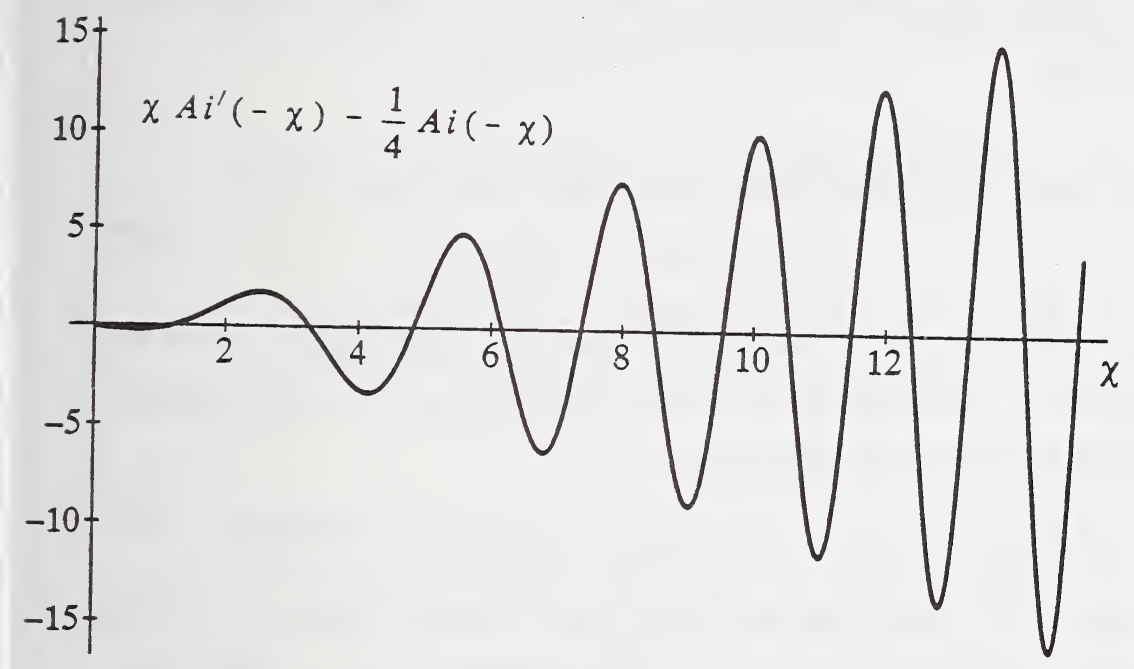

Figure 6.5 Plot of $\chi A i(\chi)-1 / 4 A i(\chi)$ as a function of $\chi$.

or

$$
\int_{0}^{x_{t}} \Gamma(x) d x=\left(\zeta_{s n}+\frac{1}{2}\right) \pi / 2,
$$

where

$$
\zeta_{s n}=\left(\frac{4}{3 \pi} Z_{s n}^{3 / 2}-1 / 2\right) .
$$

The values of $Z_{\mathrm{sn}}$ and hence $\zeta_{\mathrm{sn}}$ are universal constants, given in Table 6.2. Equation (35) is again similar to the $W K B$ quantization condition except that (for the $W K B$ case) $\zeta_{\mathrm{sn}}$ is replaced by even integers. Thus, we see that there is a clear parallel between the 
$M A F$ and the $W K B$ methodologies for a symmetric profile and the eigenvalues can be found by the $M A F$ or the $W K B$ method with equal ease.

As an example, consider the untruncated parabolic profile

$$
\Gamma^{2}(x)=\lambda^{2}-x^{2}
$$

where, following the convention of quantum mechanics, $\lambda^{2}$ is the eigenvalue. This profile is instructive because the exact and the $W K B$ eigenvalues are identical:

$$
\lambda^{2}=(2 n+1) ; \quad n=0,1,2, \ldots \quad \text { (exact and WKB). }
$$

We consider first the antisymmetric wave functions. The symmetric functions will subsequently be illustrated as well. For the antisymmetric case, the exact and the WKB wave functions are given by

$$
\begin{aligned}
& \Psi_{E X A C T}(x)=\frac{1}{2}(-1)^{(n-1) / 2} \frac{\left(\frac{n-1}{2}\right) !}{n !} \\
& \cdot H_{n}(x) \exp \left(\frac{-x^{2}}{2}\right) \quad(n=1,3,5, \ldots),
\end{aligned}
$$

where $H_{n}(x)$ are the Hermite polynomials, 


$$
\begin{gathered}
\Psi_{W K B}(x)=\frac{1}{\sqrt{\lambda}\left(\lambda^{2}-x^{2}\right)^{1 / 4}} \sin \left[\frac{\pi}{4} \lambda^{2}-\eta(x)\right] \\
\text { for } 0<x<\lambda, \\
=\frac{(-1)^{(n-1) / 2}}{2 \sqrt{\lambda}\left(x^{2}-\lambda^{2}\right)^{1 / 4}} \exp [-\zeta(x)] \quad \text { for } x>\lambda,
\end{gathered}
$$

$$
\begin{gathered}
\zeta(x)=\int_{\lambda}^{x} \sqrt{x^{2}-\lambda^{2}} d x=\frac{x}{2} \sqrt{x^{2}-\lambda^{2}} \\
-\frac{\lambda^{2}}{2} \ln \left[\frac{x+\sqrt{x^{2}-\lambda^{2}}}{\lambda}\right], \\
\eta(x)=\int_{x}^{\lambda} \sqrt{\lambda^{2}-x^{2}} d x=\frac{\pi \lambda^{2}}{4}-\frac{x}{2} \sqrt{\lambda^{2}-x^{2}} \\
\quad-\frac{\lambda^{2}}{2} \sin ^{-1}\left(\frac{x}{\lambda}\right),
\end{gathered}
$$

and the constants have been chosen to ensure that $\Psi^{\prime}(0)=1$. The $M A F$ solution is given by 


$$
\begin{gathered}
\Psi_{M A F}(x)=\frac{C\left\{\frac{3}{2} \eta(x)\right\}^{1 / 6}}{\left(\lambda^{2}-x^{2}\right)^{1 / 4}} A i\left[-\left(\frac{3}{2} \eta\right)^{2 / 3}\right] \\
\text { for } 0<x<\lambda \\
\Psi_{M A F}(x)=\frac{C\left\{\frac{3}{2} \zeta(x)\right\}^{1 / 6}}{\left(x^{2}-\lambda^{2}\right)^{1 / 4}} A i\left[\left(\frac{3}{2} \zeta\right)^{2 / 3}\right] \\
\text { for } x>\lambda,
\end{gathered}
$$

where $\eta(x)$ and $\zeta(x)$ are the same as given by Eqs. (42) and (41), respectively. The constant $C$ is given by

$$
C=\left(\frac{3 \pi}{8 \lambda}\right)^{-1 / 6}\left\{A i^{\prime}\left[-\left(\frac{3 \pi \lambda^{2}}{8}\right)^{2 / 3}\right]\right\}^{-1},
$$

which ensures that $\Psi^{\prime}(0)=1$. The wave functions for the second antisymmetric mode are plotted in Fig. 6.6a. We see excellent agreement between the exact and the $M A F$ solution but, as expected, the $W K B$ method fails near the turning points. The difference between the exact and the $M A F$ solution is so small that it is not apparent in Fig. 6.6a. We have therefore plotted the difference in Fig. 6.6b.

This untruncated parabolic profile provides an interesting case study. We have noted, for example, that the WKB method yields the exact eigenvalues. The profile is interesting for other reasons as well. The truncated parabolic profile, considered in the next 


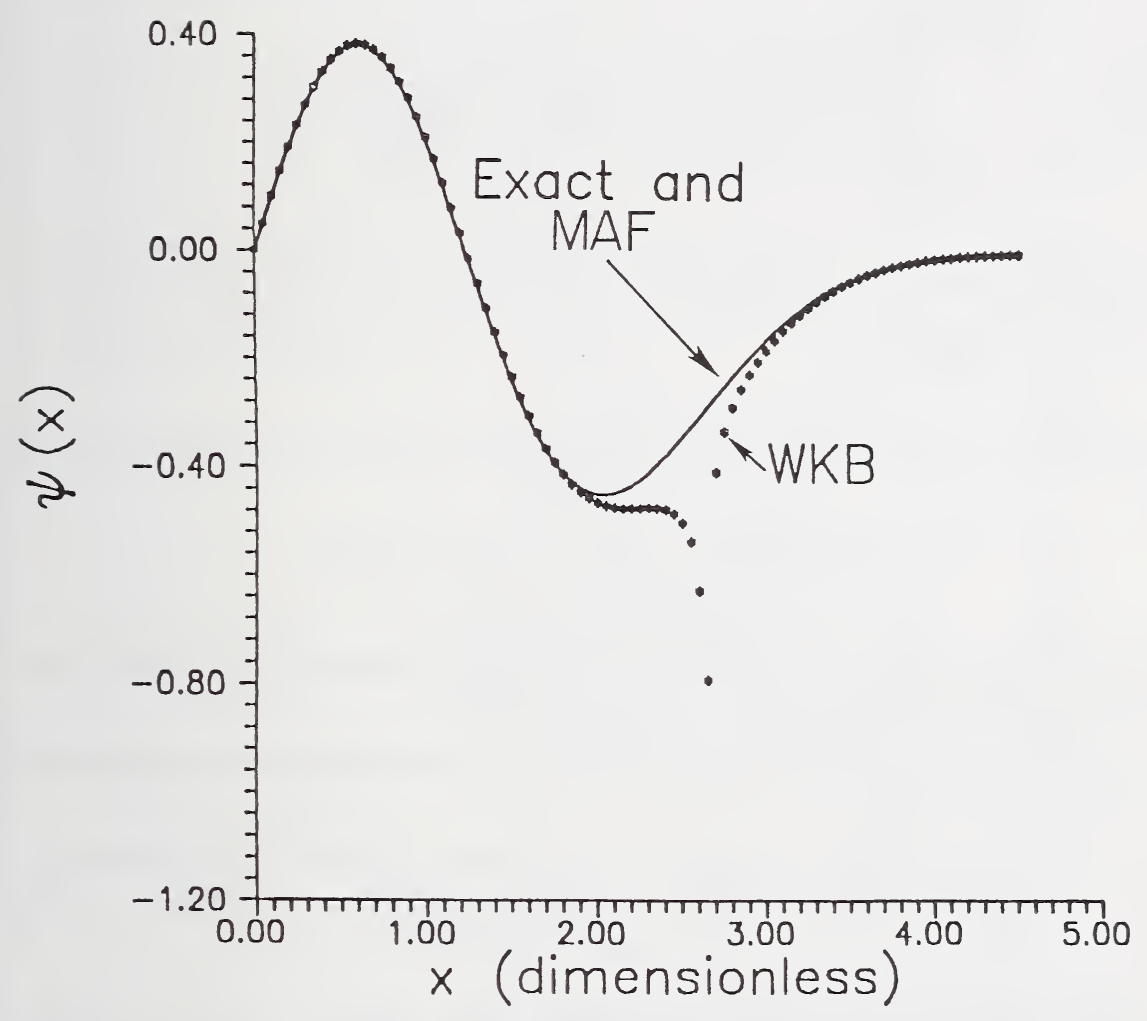

Figure 6.6(a) Exact, $W K B$ and $M A F$ wavefunctions for the optical waveguide having a symmetric profile, Example 6.2 , second antisymmetric mode.

example, is more realistic since it has the parabolic profile only in the waveguide core and is thus more representative of a geometry that is encountered in practice. The index is constant outside the core. We expect the untruncated profile to provide trend information for the truncated profile. For the truncated profile, for example, eigenfunctions and eigenvalues will begin to replicate those of the untruncated profile as the modes in question become 


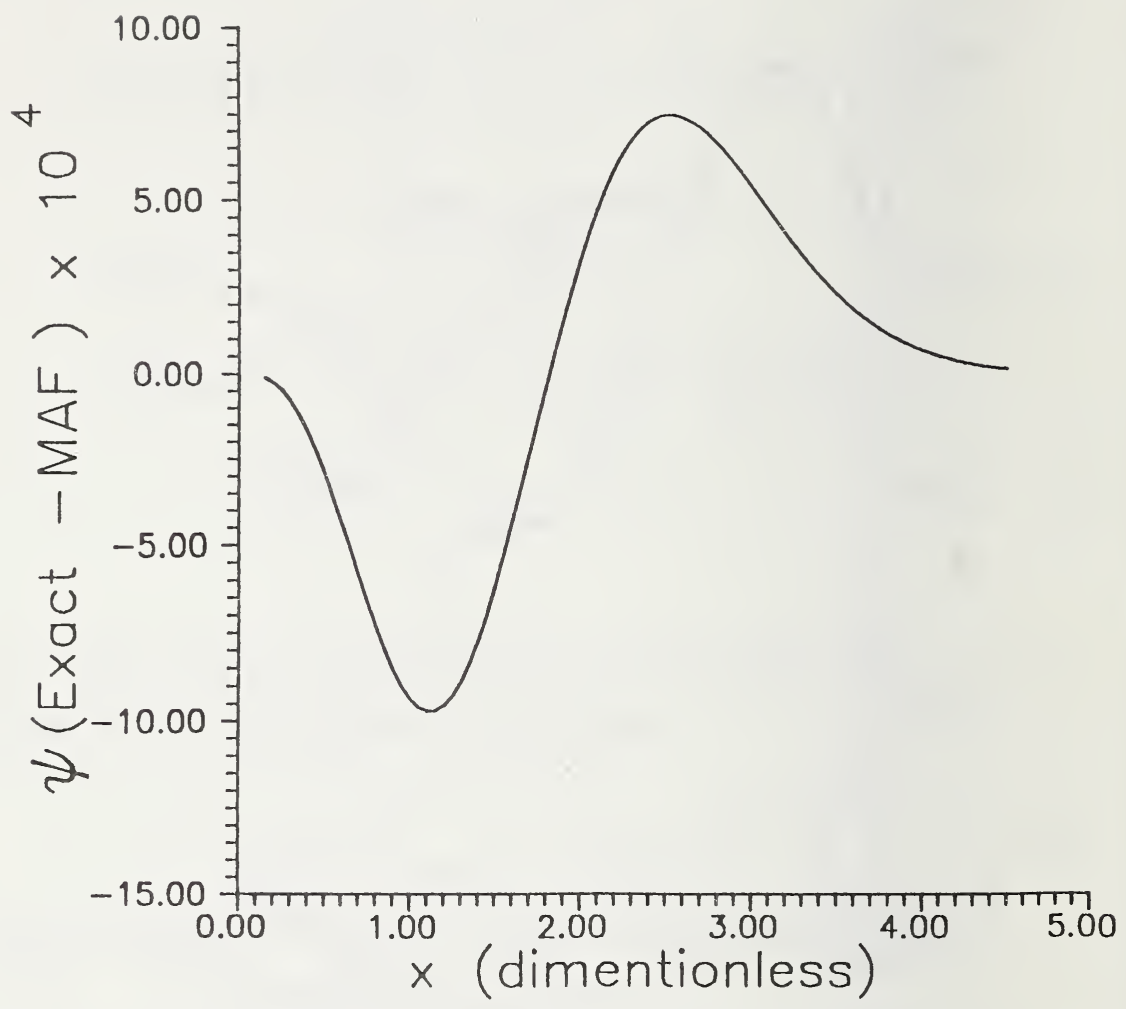

Figure 6.6(b) Difference between the exact and the MAF wavefunctions for the profile of Example 6.2, second antisymmetric mode.

more tightly bound. This will be seen in the next example.

For the symmetric case, the exact wave function is

$$
\begin{array}{r}
\Psi_{E X A C T}=(-1)^{n / 2} \frac{\left(\frac{n}{2}\right) !}{n !} H_{n} e^{-\frac{x^{2}}{2}}, \\
n=0,2,4, \ldots,
\end{array}
$$

and the MAF wave function is given by Eqs. (41) through (43) 
with $C$ given by

$$
C=\left(\frac{3 \pi}{8 \lambda}\right)^{-1 / 6}\left\{A i\left[-\left(\frac{3 \pi \lambda^{2}}{8}\right)^{2 / 3}\right]\right\}^{-1} .
$$

The value of $C$ and the constants in Eq. (45) have been chosen to ensure that $\Psi(0)=1$.

\begin{tabular}{|c|c|c||}
\hline \multicolumn{3}{|c|}{ Table 6.3} \\
Eigenvalues for the untruncated \\
parabolic profile.
\end{tabular}


The exact and $M A F$ eigenvalues are given in Table 6.3. The $W K B$ and the exact eigenvalues are the same for this profile. As expected, the accuracy of the $M A F$ method improves for the higher-order modes.

The corresponding exact and $M A F$ wave functions for the symmetric functions are plotted in figures 6.7a (for $n=0$ ) and 6.8a (for $n=4$ ). We see excellent agreement between the exact and the MAF solutions. The WKB method is not plotted but is known to fail near and at the turning points. The difference between the exact and the $M A F$ solutions is so small that it is not apparent in figures $6.7 \mathrm{a}$ and $6.8 \mathrm{a}$. We have therefore plotted the corresponding difference between the wave functions for each case in Figs. $6.7 \mathrm{~b}$ and $6.8 \mathrm{~b}$.

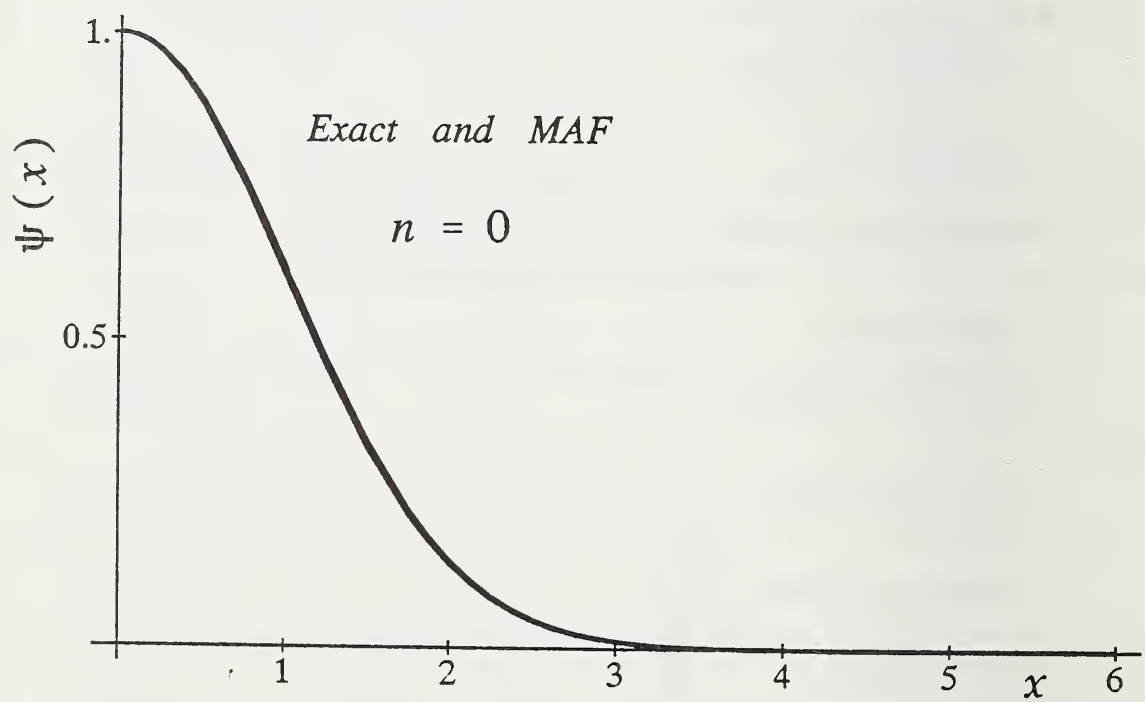

Figure 6.7(a) Exact and $M A F$ wavefunctions corresponding to $\mathrm{n}$ $=0$ in Example 6.2. 


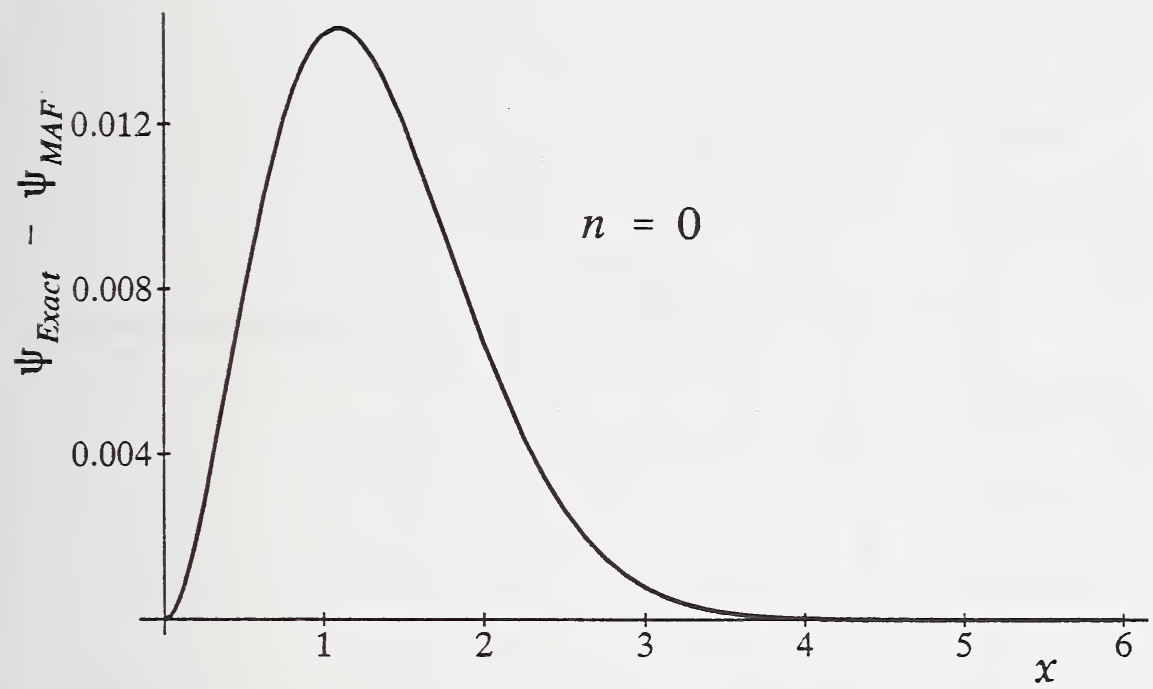

Figure 6.7(b) Difference between exact and $M A F$ wavefunctions for $n=0$ in Example 6.2.

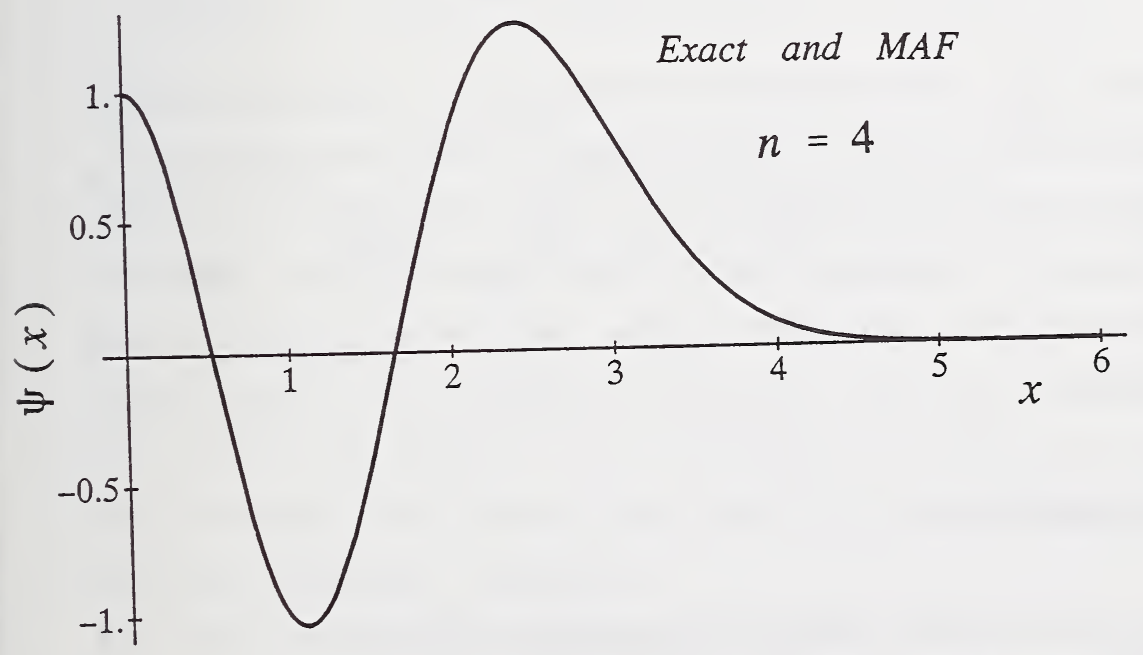

Figure 6.8(a) Exact and $M A F$ wavefunctions for $n=4$ in Example 6.2. 


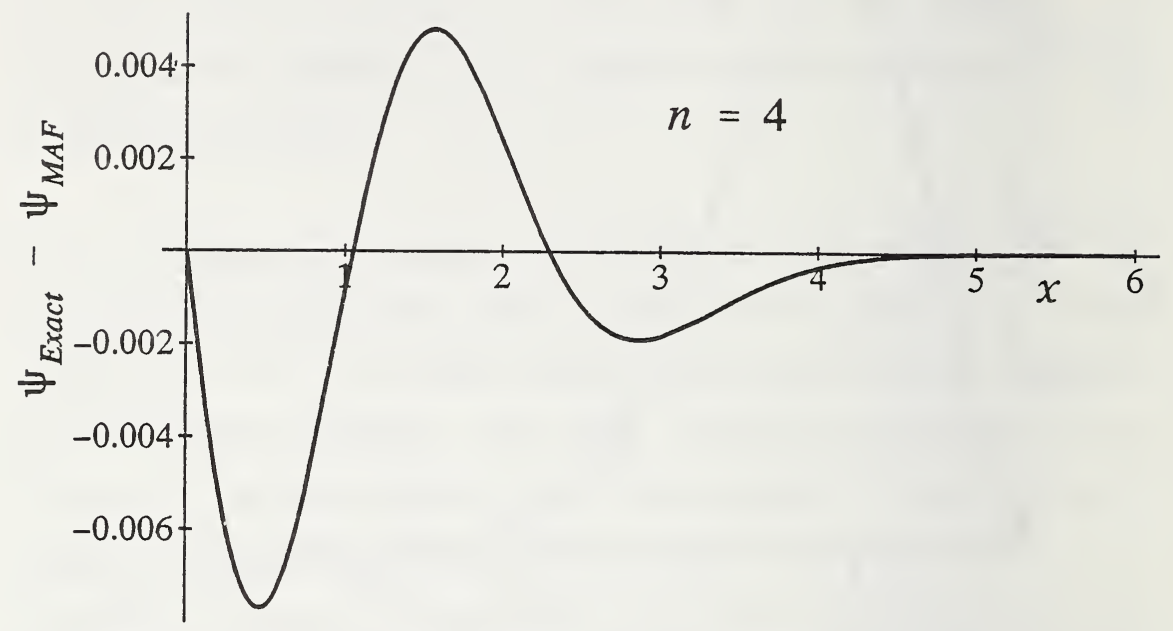

Figure 6.8(b) Difference between exact and $M A F$ wavefunctions corresponding to $n=4$ for Example 6.2.

Problem 6.1 For the profile of Example 6.2, determine the $M A F$ eigenvalues for the symmetric modes by using the values of $-Z_{\mathrm{sn}}$ as zeros of $\mathrm{Ai}^{\prime}(\chi)$ instead of $\chi \mathrm{Ai}^{\prime}(\chi)+\mathrm{Ai}(\chi) / 4$ [see Eq. (33)]. Compare the vaues so obtained with those given in Table 6.3. Why does the agreement become better with increasing mode numbers?

\section{Example 6.3}

Consider next the truncated parabolic profile shown in Fig. 6.9. For $0<x \leq a$, 


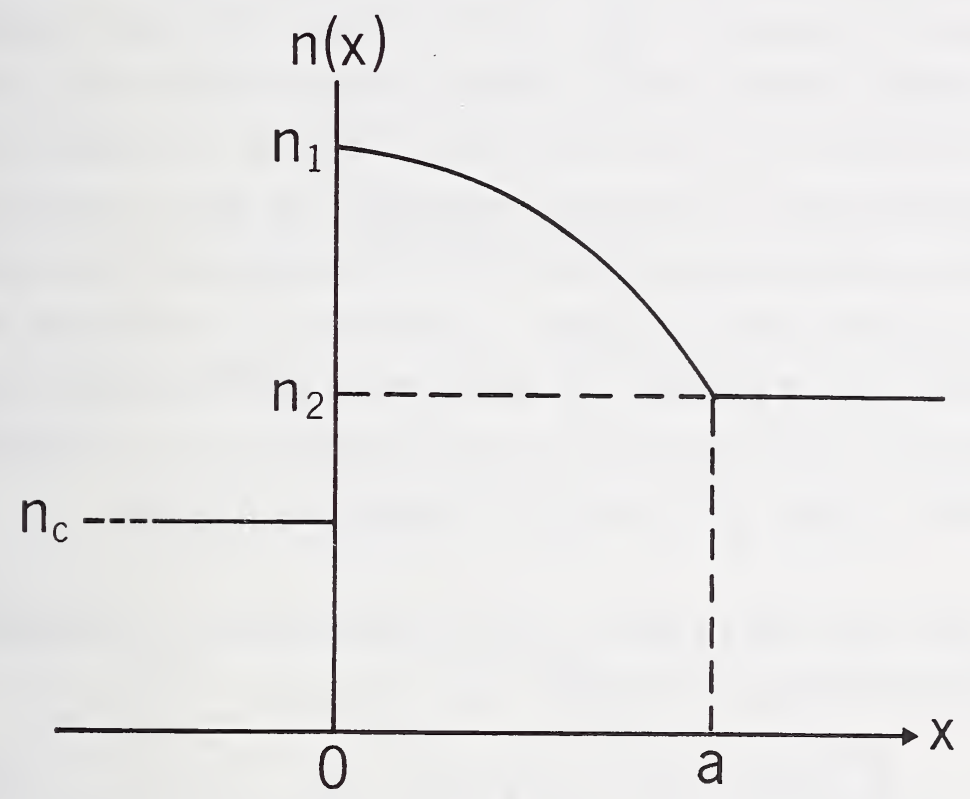

Figure 6.9 Truncated parabolic profile, Example 6.3.

$$
n(x)=\sqrt{n_{1}^{2}-\left(n_{1}^{2}-n_{2}^{2}\right)\left(\frac{x}{a}\right)^{2}}
$$

where $n_{1}$ and $n_{2}$ are as shown in the figure. $n_{\mathrm{c}}$ is the refractive index of the cover region. For $x<0$ and $x>a$, the refractive index is constant. We introduce here, for use in what follows, the normalized frequency, defined as

$$
V=\frac{2 \pi}{\lambda_{0}} a \sqrt{n_{1}^{2}-n_{2}^{2}} .
$$


This normalized frequency determines how tightly the modes are bound to the waveguide core. As $V$ increases, the modes become more tightly bound; that is, a greater fraction of the energy is in the core region $(x<a)$ of the guide. Thus, as $V$ increases, the waveguide begins to look like a waveguide that has an untrucated parabolic refractive index profile. This leads to a useful test of the approximations being suggested. If we allow $V$ to increase, the solutions that we propose (the approximate $M A F$ solutions being tested) must approach the known exact solution for the untruncated parabolic profile. If it does not, it is not very accurate.

The governing wave equation can be transformed to a convenient dimensionless form, as follows:

$$
\begin{gathered}
\frac{d^{2} \Psi(\zeta)}{d \zeta^{2}}+\left(\zeta_{0}^{2}-\alpha^{2}\right) \Psi(\zeta)=0, \quad \zeta<0, \\
\frac{d^{2} \Psi(\zeta)}{d \zeta^{2}}+\left(\zeta_{0}^{2}-\zeta^{2}\right) \Psi(\zeta)=0,0<\zeta<\sqrt{V}, \\
\frac{d^{2} \Psi(\zeta)}{d \zeta^{2}}+\left(\zeta_{0}^{2}-V\right) \Psi(\zeta)=0, \sqrt{V} \leq \zeta,
\end{gathered}
$$

where

$$
\zeta=\sqrt{V} \frac{x}{a},
$$




$$
\begin{gathered}
\alpha^{2}=V \frac{n_{1}^{2}-n_{c}^{2}}{n_{1}^{2}-n_{2}^{2}}, \\
\zeta_{0}^{2}=V(1-b) .
\end{gathered}
$$

$x=a$ corresponds to $\zeta=V^{1 / 2}$. The solutions to Eqs. (49) and (51) are

$$
\psi(\zeta)=\exp \left(\sqrt{\alpha^{2}-\zeta_{0}^{2}} \zeta\right), \quad \zeta \leq 0,
$$

and

$$
\Psi(\zeta)=c_{3} \exp \left(-\sqrt{V-\zeta_{0}^{2}} \zeta\right), \quad \zeta \geq \sqrt{V}
$$

The exponentially diverging solutions have been neglected to ensure that $\Psi \rightarrow 0$ as $x$ (and hence $\zeta$ ) $\rightarrow \pm \infty$. The multiplying constant in Eq. (55) is taken to be unity so that $\Psi(0)=1$.

The exact solution in the nonuniform region, where the refractive index is a parabolic profile can be written in terms of the HermiteGauss functions. Exact evaluation and analysis then requires that the boundary conditions be applied at the two boundaries with the uniform regions, wherein the solutions are decaying exponential functions. We will not evaluate the exact solution, but rely instead on a simple matrix method, for which the numerical precision is well established [Ghatak, Thyagarajan, and Shenoy, (1987)]. In the numerical results to be given later, then, comparison of the $M A F$ method will be with the matrix method. 
Turning to the $M A F$ solution, we note first that the solutions given by Eqs. (55) and (56) are again valid outside the core region where the refractive index is constant. In the core of the waveguide $(0 \leq \zeta \leq \sqrt{ } V)$, we take

$$
\psi(\zeta)=\frac{1}{\sqrt{\xi^{\prime}}}\left[C_{1} A i(\xi)+C_{2} B i(\xi)\right],
$$

where, for $0<\zeta<\zeta_{0}$,

$$
\begin{aligned}
\xi(\zeta)=- & {\left[\frac { 3 } { 2 } \left(\frac{\pi \zeta_{0}^{2}}{4}-\frac{\zeta}{2} \sqrt{\zeta_{0}^{2}-\zeta^{2}}-\frac{\zeta_{0}^{2}}{2}\right.\right.} \\
& \left.\left.\cdot \sin ^{-1}\left[\frac{\zeta}{\zeta_{0}}\right]\right)\right]^{\frac{2}{3}},
\end{aligned}
$$

and for $\zeta_{0} \leq \zeta \leq V^{1 / 2}$,

$$
\xi(\zeta)=\left[\frac{3}{2}\left(\frac{\zeta}{2} \sqrt{\zeta^{2}-\zeta_{0}^{2}}-\frac{\zeta_{0}^{2}}{2} \ln \frac{\zeta+\sqrt{\zeta^{2}-\zeta_{0}^{2}}}{\zeta_{0}}\right)\right]^{\frac{2}{3}} .
$$

The boundary conditions require that $\Psi(\zeta)$ and $\Psi^{\prime}(\zeta)$ be continuous at $\zeta=0$ and at $\zeta=V^{1 / 2}$ (that is at $x=0$ and $x=$ a). Applying these conditions, we get the required eigenvalue equation: 


$$
\frac{A i^{\prime}\left(\xi_{0}\right)-\gamma_{0} A i\left(\xi_{0}\right)}{A i^{\prime}\left(\xi_{1}\right)-\gamma_{1} A i\left(\xi_{1}\right)}=\frac{B i^{\prime}\left(\xi_{0}\right)-\gamma_{0} B i\left(\xi_{0}\right)}{B i^{\prime}\left(\xi_{1}\right)-\gamma_{1} B i\left(\xi_{1}\right)},
$$

where

$$
\begin{aligned}
& \gamma_{0}=\frac{\xi_{0}^{\prime \prime}}{2\left(\xi_{0}^{\prime}\right)^{2}}+\frac{\sqrt{\alpha^{2}-\zeta_{0}^{2}}}{\xi_{0}^{\prime}}, \\
& \gamma_{1}=\frac{\xi_{1}^{\prime \prime}}{2\left(\xi_{1}^{\prime}\right)^{2}}-\frac{\sqrt{V-\zeta_{0}^{2}}}{\xi_{1}^{\prime}},
\end{aligned}
$$

and subscripts 0 and 1 refer to values at $\zeta=0$ and $\zeta=V^{1 / 2}$. Solution of Eq. (60) yields the normalized propagation constant $b$ as desired.

As in the previous example, the $M A F$-predicted electromagnetic field for this profile is again extremely accurate and for brevity we have not plotted it here. We will concentrate instead on the accuracy of the normalized propagation constant $b$. This is an interesting example in this respect because the WKB method gives the exact eigenvalue when the parabolic profile is untruncated. The $M A F$ method will therefore give very accurate results for large $V$.

Figure 6.10 shows $\Delta b / b$ for this truncated profile where $\Delta b$ is the difference in the values of $b$ using the exact (as determined by the matrix method) and $M A F$ method or the difference between the $W K B$ and the exact values. We see that the MAF method is more accurate than the WKB method at low values of $V$, but for high $V$ values, where the errors are small for either method, the WKB 


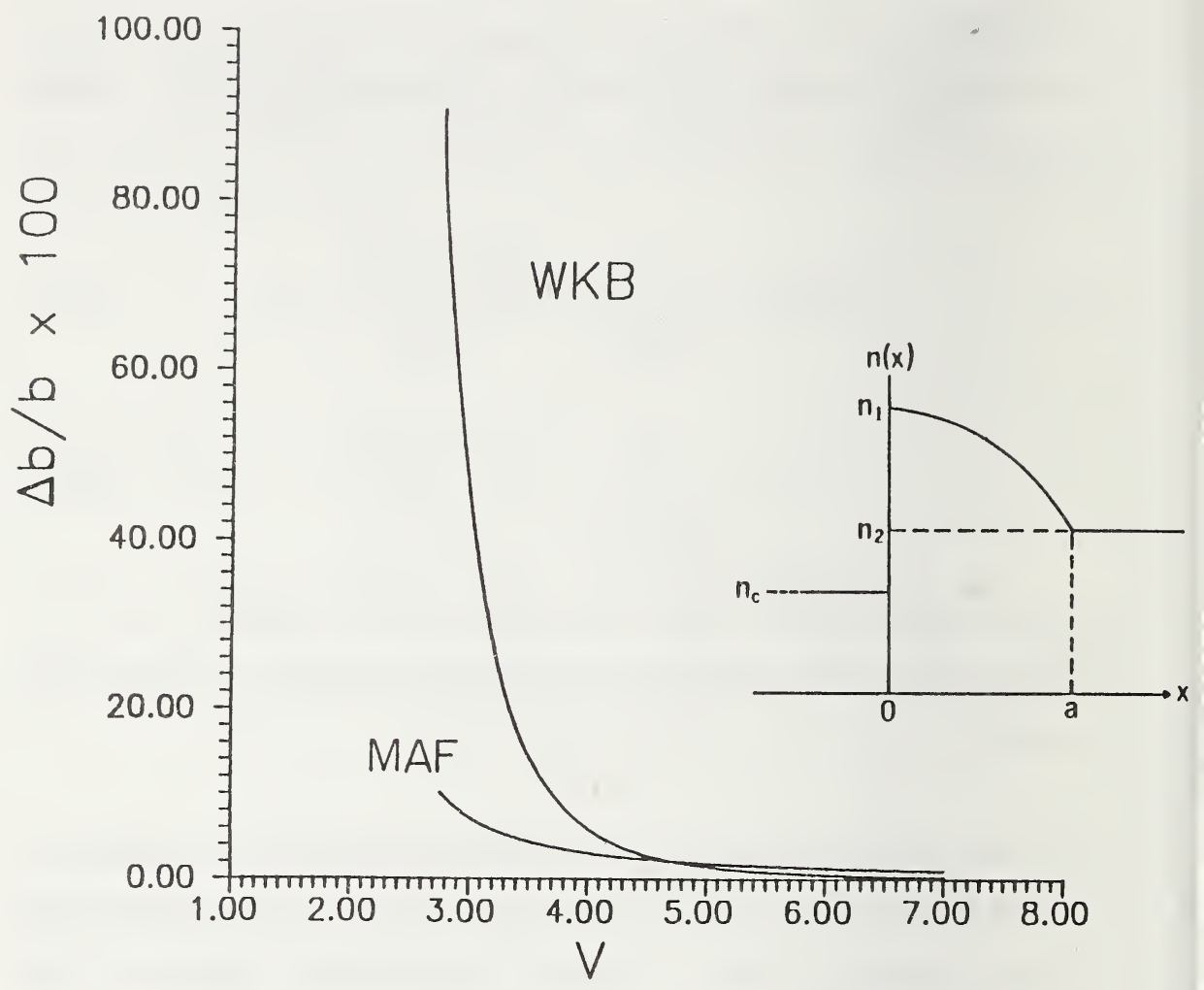

Figure 6.10 Fractional error in MAF normalized propagation constant for the truncated parabolic profile.

results are more accurate. This is undoubtedly related to the fact that $W K B$ yields exact eigenvalues for the infinite (untruncated) parabolic profile.

\section{Example 6.4}

In this example, the $M A F$ technique is extended to profiles which 
are not symmetric in $x$. Without loss of generality, we assume that in every case, $\Gamma^{2}(x)$ will have a maximum which we take to be at $x=0$. Let

$$
\begin{aligned}
& \Gamma^{2}(x)=\Gamma_{1}^{2}(x) \quad \text { for } x>0, \\
& \Gamma^{2}(x)=\Gamma_{2}^{2}(x) \text { for } x<0 .
\end{aligned}
$$

Then

$$
\Psi_{M A F}(x)= \begin{cases}\sqrt{\frac{\xi_{1}^{\prime}(0)}{\xi_{1}^{\prime}(x)} \frac{A i\left(\xi_{1}(x)\right)}{A i\left(\xi_{1}(0)\right)}} & \text { for } x>0 \\ \sqrt{\frac{\xi_{2}^{\prime}(0)}{\xi_{2}^{\prime}(x)}} \frac{A i\left(\xi_{2}(x)\right)}{A i\left(\xi_{2}(0)\right)} & \text { for } x<0,\end{cases}
$$

where

$$
\begin{array}{ll}
\xi_{1}(x)=\left\{\frac{3}{2} \int_{x_{t 1}}^{x} \sqrt{-\Gamma_{1}^{2}} d x\right\}^{2 / 3} \quad 0 \leq x<\infty, \\
\xi_{2}(x)=\left\{\frac{3}{2} \int_{x_{t 2}}^{x} \sqrt{-\Gamma_{2}^{2} d x}\right\}^{2 / 3} \quad-\infty<x \leq 0,
\end{array}
$$

and now $x=x_{\mathrm{t} 1}$ and $x=x_{\mathrm{t} 2}$ are turning points, one in the region $x>0$ and the other in the region $x<0$. Continuity of $\Psi$ and $\Psi^{\prime}$ at $x=0$ will give the following transcendental equation, from which the eigenvalues can be determined: 


$$
\begin{aligned}
& \frac{A i^{\prime}\left(\xi_{1}(0)\right)}{A i\left(\xi_{1}(0)\right)} \xi_{1}^{\prime}(0)-\frac{1}{2} \frac{\xi_{1}^{\prime \prime}(0)}{\xi_{1}^{\prime}(0)} \\
= & \frac{A i^{\prime}\left(\xi_{2}(0)\right)}{A i\left(\xi_{2}(0)\right)} \xi_{2}^{\prime}(0)-\frac{1}{2} \frac{\xi_{2}^{\prime \prime}(0)}{\xi_{2}^{\prime}(0)} .
\end{aligned}
$$

We now consider the asymmetric profile:

$$
\begin{gathered}
\Gamma_{1}^{2}(x)=a+b \exp \left(-\frac{x}{d}\right)-\gamma, \quad x>0, \\
\Gamma_{2}^{2}(x)=a+b-\gamma+\frac{b x}{2 d}, \quad x<0,
\end{gathered}
$$

where $a$ and $b$ are constants and $\gamma$ is the eigenvalue. The exact solution for this profile is

$$
\Psi_{E X A C T}(x)=\left\{\begin{array}{cc}
\frac{A i\left(z_{1}-b_{1}^{1 / 3} x\right)}{A i\left(z_{1}\right)} & x<0 \\
\frac{J_{v}\left[z_{2} \exp \left(-\frac{x}{2 d}\right)\right]}{J_{v}\left(z_{2}\right)} & x>0,
\end{array}\right.
$$

where $a_{1}=a+b-\gamma, b_{1}=b / 2 d, v=2 d(\gamma-a)^{1 / 2}, z_{1}=$ $a_{1} / b_{1}^{2 / 3}, z_{2}=2 d(b)^{1 / 2}$, and the multiplying constant has been chosen to ensure that $\Psi_{\text {EXACT }}(0)=1$. Continuity of $\Psi$ and $\Psi^{\prime}$ at $x=0$ yields 


$$
\frac{A i^{\prime}\left(z_{1}\right)}{A i\left(z_{1}\right)}\left(b_{1}\right)^{1 / 3}=\frac{\sqrt{b}}{2} \frac{J_{v-1}\left(z_{2}\right)-J_{v+1}\left(z_{2}\right)}{J_{v}\left(z_{2}\right)} .
$$

From this we can find the eigenvalues. The corresponding eigenfunctions can be obtained from Eq. (70). The WKB eigenvalues are found from

$$
\begin{aligned}
\int_{x_{t 2}}^{0} \Gamma_{2}(x) d x+\int_{0}^{x_{t 1}} \Gamma_{1}(x) d x & =\left(m+\frac{1}{2}\right) \pi, \\
m & =0,1,2, \ldots .
\end{aligned}
$$

where $x_{\mathrm{t} 1}$ and $x_{\mathrm{t} 2}$ are the turning points. For this profile, Eq. (72) simplifies to

$$
\begin{gathered}
\frac{2}{3 b_{1}} a_{1}^{3 / 2}+2 d\left[\sqrt{a_{1}}-\sqrt{\gamma-a} \tan ^{-1}\left(\frac{a_{1}}{\gamma-a}\right)^{1 / 2}\right] \\
=\left(m+\frac{1}{2}\right) \pi .
\end{gathered}
$$

From Eqs. (65), (66), (68), and (69), we obtain

$$
\begin{gathered}
\xi_{1}(x)=-(3 d)^{2 / 3}\left[\left(b \exp \left(-\frac{x}{d}\right)+a-\gamma\right)^{1 / 2}\right. \\
\left.-\sqrt{\gamma-a} \tan ^{-1}\left(\frac{b \exp (-x / d)}{\gamma-a}-1\right)^{1 / 2}\right]^{2 / 3} ; \\
x<x_{t 1},
\end{gathered}
$$




$$
\begin{gathered}
\xi_{1}(x)=(3 d)^{2 / 3}\left[-\left[\gamma-a-b \exp \left(-\frac{x}{d}\right)\right]^{1 / 2}\right. \\
\left.+\frac{\sqrt{\gamma-a}}{2} \ln \frac{\left(1+\sqrt{1-\frac{b e^{-x / d}}{\gamma-a}}\right)}{\left(1-\sqrt{1-\frac{b e^{x / d}}{\gamma-a}}\right)}\right]^{2 / 3}, x>x_{t 1}, \\
\xi_{2}(x)=-\frac{a_{1}+b_{1} x}{b_{1}^{2 / 3}} .
\end{gathered}
$$

Equations (67) and (64) yield the eigenvalues and eigenfunctions using the $M A F$ method. We used typical values of the parameters in the profile of Eqs. (68) and (69). We used $a=4, b=0.2$, and $d=10$. The values of $\gamma$ for the first two modes are given in Table 6.4.

The MAF method yields values correct to one part in $10^{5}$ and are more accurate than those found by WKB.

Now, define a normalized eigenvalue as 


$$
\Lambda=\frac{\gamma-a}{b}
$$

Since the bound state eigenvalues necessarily lie between $(a+b)$ and $a$, the values of $\Lambda$ are more meaningful to compare than the values of $\gamma$. The values of $\Lambda$ for the first two states are given in Table 6.5. Again, the values calculated by the MAF method are much more accurate than those from the WKB method. Figure 6.11a gives a plot of $\Psi(x)$ for the exact and the $M A F$ solutions corresponding to the first mode. We have plotted the field only for positive $x$.

\begin{tabular}{||c|c|c|c||}
\hline \multicolumn{4}{|c||}{ Table 6.4 } \\
$\gamma$ values for first two modes. \\
\hline & EXACT & MAF & WKB \\
\hline$\gamma_{1}$ & 4.144976 & 4.145002 & 4.139262 \\
\hline$\gamma_{2}$ & 4.079228 & 4.079254 & 4.079617 \\
\hline
\end{tabular}

\begin{tabular}{||c|c|c|c||}
\hline \multicolumn{5}{|c||}{$\Lambda$ Table 6.5} \\
& EXACT & MAF & WKB \\
\hline$\Lambda_{1}$ & 0.72488 & 0.72501 & 0.69631 \\
\hline$\Lambda_{2}$ & 0.39614 & 0.39627 & 0.39808 \\
\hline
\end{tabular}


The $M A F$ and the exact solutions are identical for negative $x$ because the profile $\left(\Gamma^{2}(x)\right.$ is linear there. Since the difference between the two solutions is not apparent in Fig. 6.11a, we have plotted the difference in Fig. 6.11b. $\Psi_{\text {WKB }}$ has not been plotted because it is known to fail near the turning points.

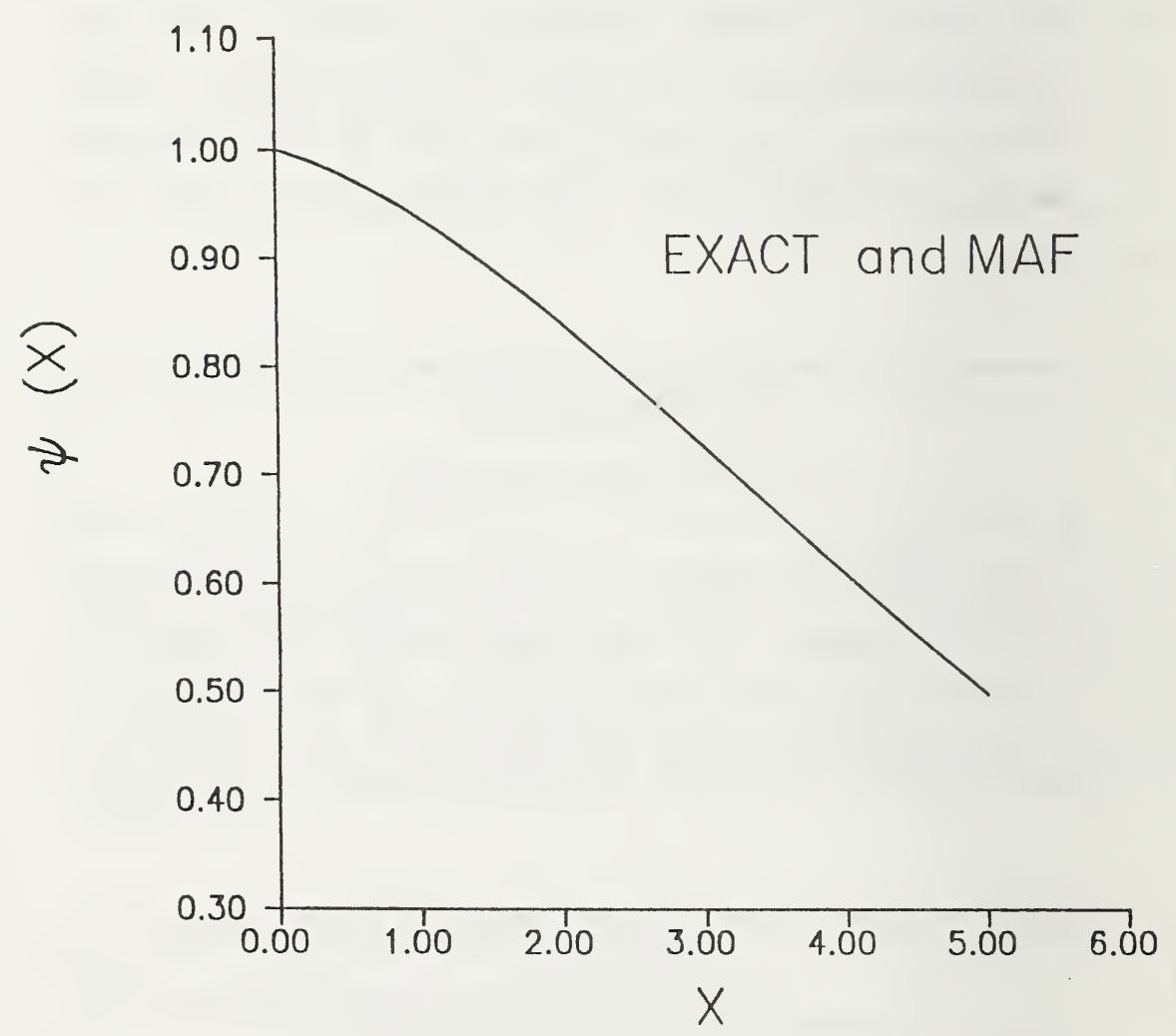

Figure 6.11(a). Exact and MAF wavefunction for the first mode, Example 6.4. 


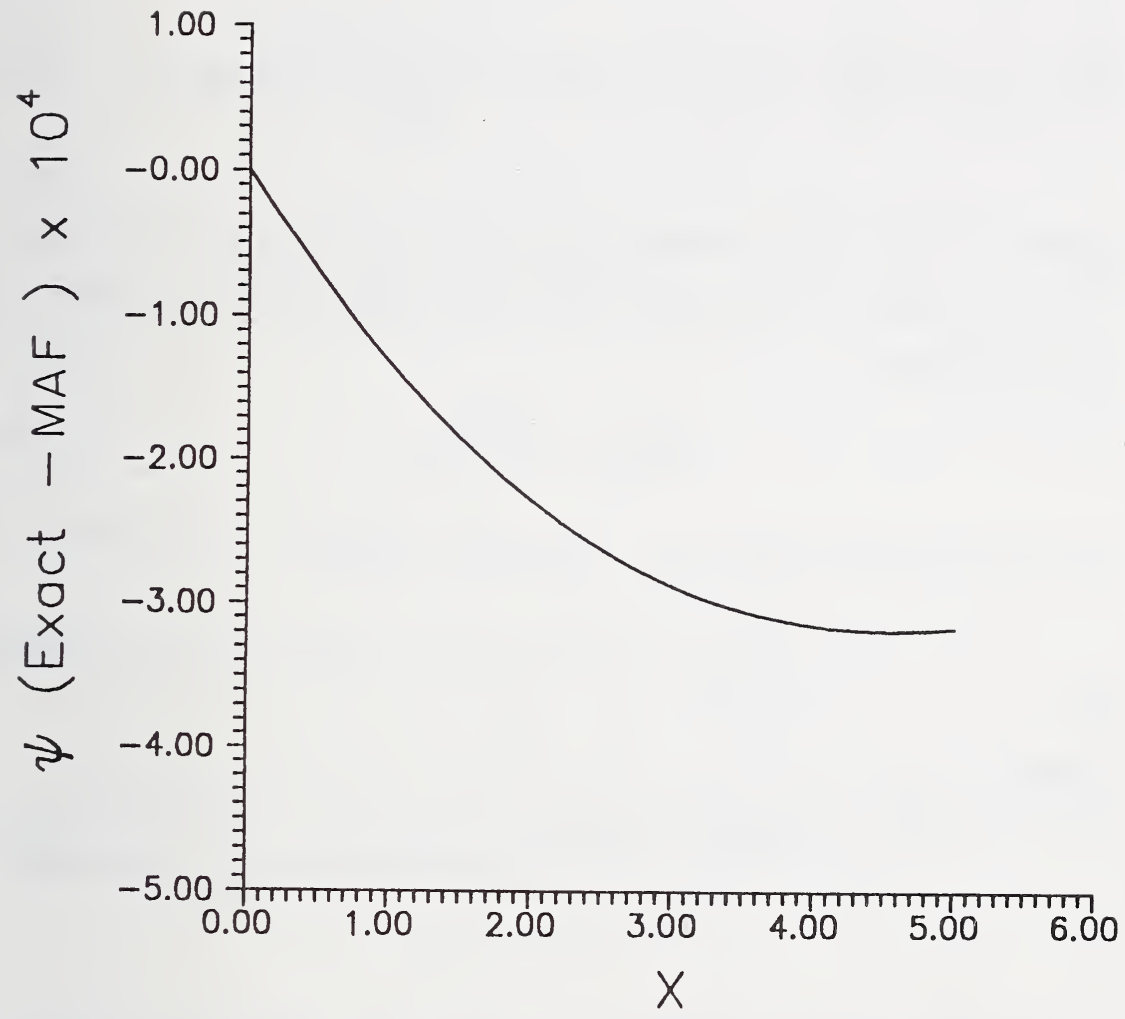

Figure 6.11(b) Difference between the exact and $M A F$ wavefunctions for the first mode, Example 6.4 .

\section{Example 6.5}

For a spherically symmetric potential $V(r)$ the radial part of the wave function $R(r)$ satisfies Schrödinger's wave equation 


$$
\frac{1}{r^{2}} \frac{d}{d r}\left[r^{2} \frac{d R}{d r}\right]+\frac{2 \mu}{\hbar^{2}}\left[E-V(r)-\frac{\ell(\ell+1) \hbar^{2}}{2 \mu r^{2}}\right] R(r)=0,
$$

where $E$ is the energy eigenvalue and the other symbols have their usual meaning [Ghatak and Lokanathan (1984)]. If we make the transformation

$$
R(r)=\frac{\Psi(r)}{r},
$$

and consider the $\ell=0$ case, we readily obtain

$$
\frac{d^{2} \psi}{d r^{2}}+\Gamma^{2}(r) \psi(r)=0,
$$

where

$$
\Gamma^{2}(r)=\frac{2 \mu}{\hbar^{2}}[E-V(r)] .
$$

Following the results of Chapter 4, we obtain the following $M A F$ solution to Eq. (80):

$$
\psi(r)=C_{1} \frac{A i[\xi(r)]}{\sqrt{\xi^{\prime}(r)}},
$$

where

$$
\xi(r) \equiv\left\{\frac{3}{2} \int_{r_{t}}^{r} \sqrt{-\Gamma^{2}(r)} d r\right\}^{\frac{2}{3}},
$$

and $r=r_{\mathrm{t}}$ represents the turning point $\left(\Gamma\left(r_{\mathrm{t}}\right)=0\right)$. We have neglected the $B i$ solution, which diverges at $r=\infty$. The quantity $\xi$ [as defined through Eq. (83)] is negative for $r<r_{\mathrm{t}}$ [where $\Gamma^{2}(\mathrm{r})$ is positive] and positive for $r>r_{\mathrm{t}}$ (where $\Gamma^{2}(\mathrm{r})$ is negative). Thus 


$$
\xi(r)=-\left\{\frac{3}{2} \int_{r}^{r_{t}} \Gamma(r) d r\right\}^{\frac{2}{3}}, \quad 0<r<r_{t},
$$

and

$$
\xi(r)=\left\{\frac{3}{2} \int_{r_{t}}^{r} \kappa(r) d r\right\}^{\frac{2}{3}}, \quad r>r_{t},
$$

where

$$
\kappa^{2}(r)=-\Gamma^{2}(r)
$$

is positive in the region $r>r_{\mathrm{t}}$.

Because of Eq. (79),

$$
\psi(0)=0 .
$$

The eigenvalues are therefore obtained from the solution of the following transcendental equation

$$
A i[\xi(0)]=0 .
$$

Following the discussion of example 6.2 [see Eq. (24)], the MAF eigenvalues are found from the following equations:

$$
\int_{0}^{r_{t}} \Gamma(r) d r=\left(\zeta_{a n}+\frac{1}{2}\right) \frac{\pi}{2},
$$

where $\zeta_{\text {an }}$, given in the third column of Table 6.2, are nearly odd integers. The reason is seen from the following: Recall that for antisymmetric modes corresponding to a symmetric onedimensional potential,

$$
\Psi(0)=0,
$$


and the corresponding WKB quantization condition [see Eq. (26) of Chapter 5]

$$
\int_{-x_{t}}^{+x_{t}} \Gamma(x) d x=\left(n+\frac{1}{2}\right) \pi \quad n=1,3,5, \ldots,
$$

simplifies to

$$
\int_{0}^{x_{t}} \Gamma(x) d x=\left(n+\frac{1}{2}\right) \frac{\pi}{2} .
$$

Thus, Eq. (89) is expected to have approximately the same accuracy as the $W K B$ analysis. In fact, for the potential function considered next, our Eq. (89) gives an error in the eigenvalue of 0.84 percent, whereas the WKB quantization condition gives an error of 3.2 percent.

Consider now the profile

$$
V(r)=-V_{o} \exp \left(-\frac{r}{a}\right) .
$$

The exact eigenvalues are obtained by solving the transcendental equation [Ghatak and Lokanathan (1984)]:

$$
J_{v}(g)=0,
$$

where 


$$
\nu=\left[-\frac{8 \mu E a^{2}}{\hbar^{2}}\right]^{\frac{1}{2}} ; \quad g=\left[\frac{8 \mu V_{0} a^{2}}{\hbar^{2}}\right]^{\frac{1}{2}}
$$

Consider the deuteron problem, for which $V_{\mathrm{o}} \approx 40 \mathrm{MeV} \approx 6.409$ x $10^{-12} \mathrm{~J}, a \approx 1.85 \times 10^{-15} \mathrm{~m}, \mu \approx 8.398 \times 10^{-22} \mathrm{~kg}$ ( $\mu$ represents the reduced mass), so $g \approx 3.64$.

Equation (93) gives $v=0.858628$. The corresponding value of $\checkmark$ obtained from Eq. (89) is 0.865815 . In Chapter 7 we will revisit this problem and show how the well-known perturbation theory, used in conjunction with the $M A F$ solutions, gives results that agree extremely well with the exact result. The corresponding $W K B$ value is given by $v_{\mathrm{WKB}}=0.886341$. Using $v=$ 0.858628 , we get $E=2.223 \mathrm{MeV}$, which represents the correct binding energy of the deuteron.

Figure 6.12 shows the exact wave function along with the WKB and $M A F$ solution. Not surprisingly, the WKB solution fails at the turning point but the $M A F$ solution agrees well with the exact solution throughout the region of interest. Because of the good agreement, we also show, in Fig. 6.13, the difference between the exact and the $M A F$ solutions.

For $g=3.64$, there is only one bound state. If there are more bound states, the $M A F$ solutions are more accurate for the higherorder states.

We have also analyzed the three-dimensional harmonic oscillator 


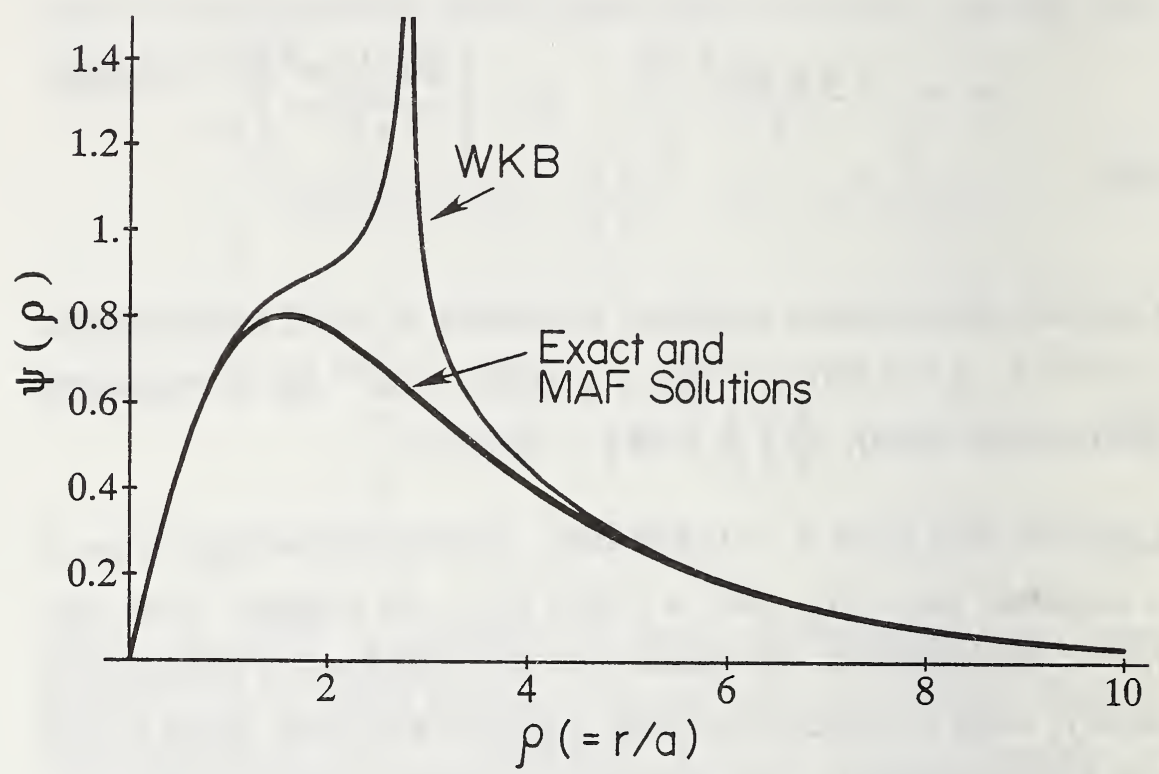

Figure 6.12 Exact, $W K B$, and $M A F$ solutions to Schrödinger's equation $(\ell=0)$ for the potential function of Eq. (92).

and found excellent agreement between the $M A F$ solution and the exact wave functions. Unfortunately, for the Coulomb potential the eigenvalues found from the $M A F$ method for the first two or three modes are in considerable error. This may be due to the singularity of the potential at $r=0$.

\subsection{Conclusions}

In this chapter, we have shown that the $M A F$ solution gives extremely accurate wave functions for problems of practical interest and should be of considerable use in many cases. Unlike the WKB solutions, the MAF solutions hold throughout the region 


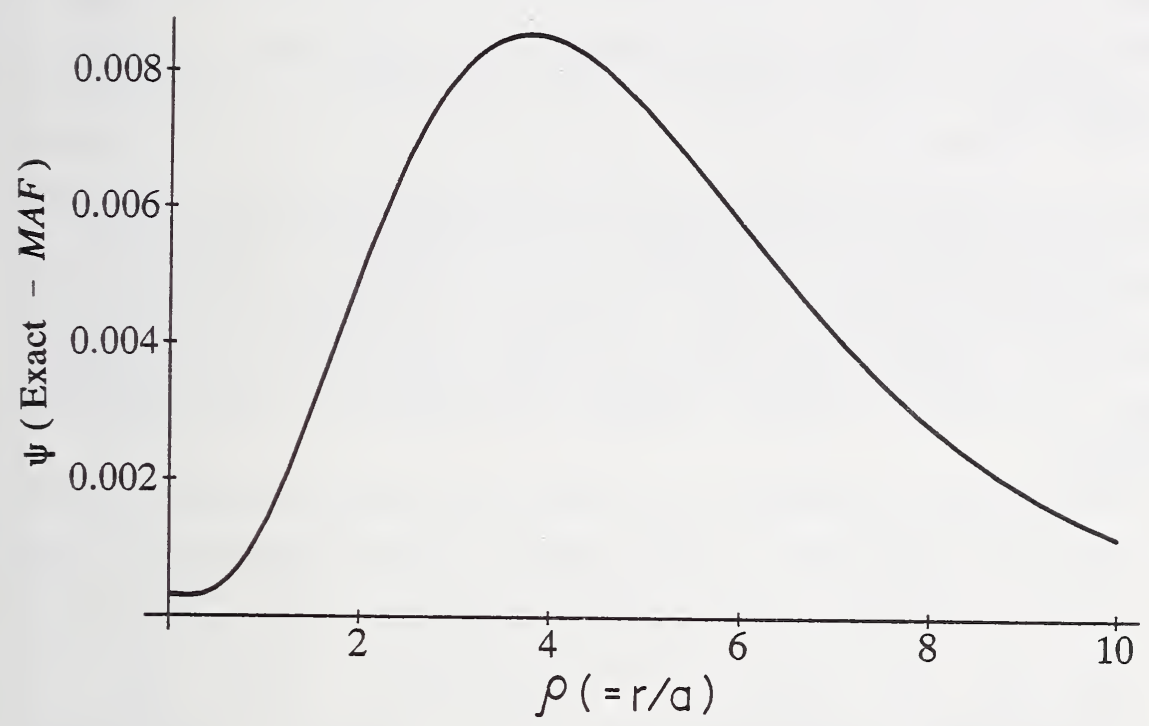

Figure 6.13 Difference between the exact and the MAF wavefunctions corresponding to Fig. 6.12.

of interest, including the turning point.

In Examples 6.2 and 6.5, we gave a simple analogy to the wellknown WKB quantization condition. Our analogy holds provided that the profile is symmetric. In particular, we showed that the eigenvalue equation takes the familiar form

$$
\int_{0}^{r_{t}} \Gamma(r) d r=\left(\zeta_{n}+\frac{1}{2}\right) \frac{\pi}{2},
$$


where $r_{\mathrm{t}}$ is the turning point. The values of $\zeta_{\mathrm{n}}$ are universal constants and are given in Table 6.2, where they are labeled $\zeta_{\text {an }}$ and $\zeta_{\mathrm{sn}}$ to denote antisymmetric and symmetric modes, respectively.

The form of Eq. (95) suggests that the result gives accuracy of about the same order as the WKB method. For Example 6.5, our accuracy is considerably better than that of the WKB method; the error is about 3.2 percent $(W K B)$ as opposed to 0.84 percent (MAF). 


\section{EXTENSION OF MAF METHOD FOR EIGENVALUES}

\subsection{Introduction}

In this chapter we derive and use an improvement to the $M A F$ method; it is based on well-known perturbation techniques. The method dramatically improves the accuracy of the eigenvalues and hence represents a powerful tool for use in those special cases when improved accuracy is required. The formulation follows closely the paper by Goyal, Gallawa, and Ghatak (1991c).

We will first demonstrate the method by examining a nonuniform planar waveguide having a profile that is amenable to exact solution. The profile was used in Example 6.1 of Chapter 6. We will then further demonstrate the concept by applying Schrödinger's equation to a three-dimensional symmetrical potential well. This example was also considered in Chapter 6 . In this chapter, we will discuss only the determination of eigenvalues.

\subsection{Formulation and Example 7.1}

Consider the scalar wave equation appropriate to a twodimensional optical waveguide,

$$
\frac{d^{2} \psi}{d x^{2}}+\Gamma^{2}(x) \psi(x)=0,
$$


where $\Gamma^{2}(x)=k_{0}{ }^{2} n^{2}(x)-\beta^{2}, k_{0}$ is the free-space wavenumber, $n(x)$ is the $x$-dependent refractive index variation, and $\beta$ is the propagation constant. We consider the following index profile:

$$
n^{2}(x)= \begin{cases}n_{2}^{2}+\left(n_{1}^{2}-n_{2}^{2}\right) \exp \left(-\frac{x}{d}\right) & \text { for } x>0 \\ n_{c}^{2} & \text { for } x<0,\end{cases}
$$

where $n_{1}, n_{2}, n_{\mathrm{c}}$ are the refractive indices shown in Fig. 7.1. We can use Eq. (2) to rewrite Eq. (1) in normalized form as follows:

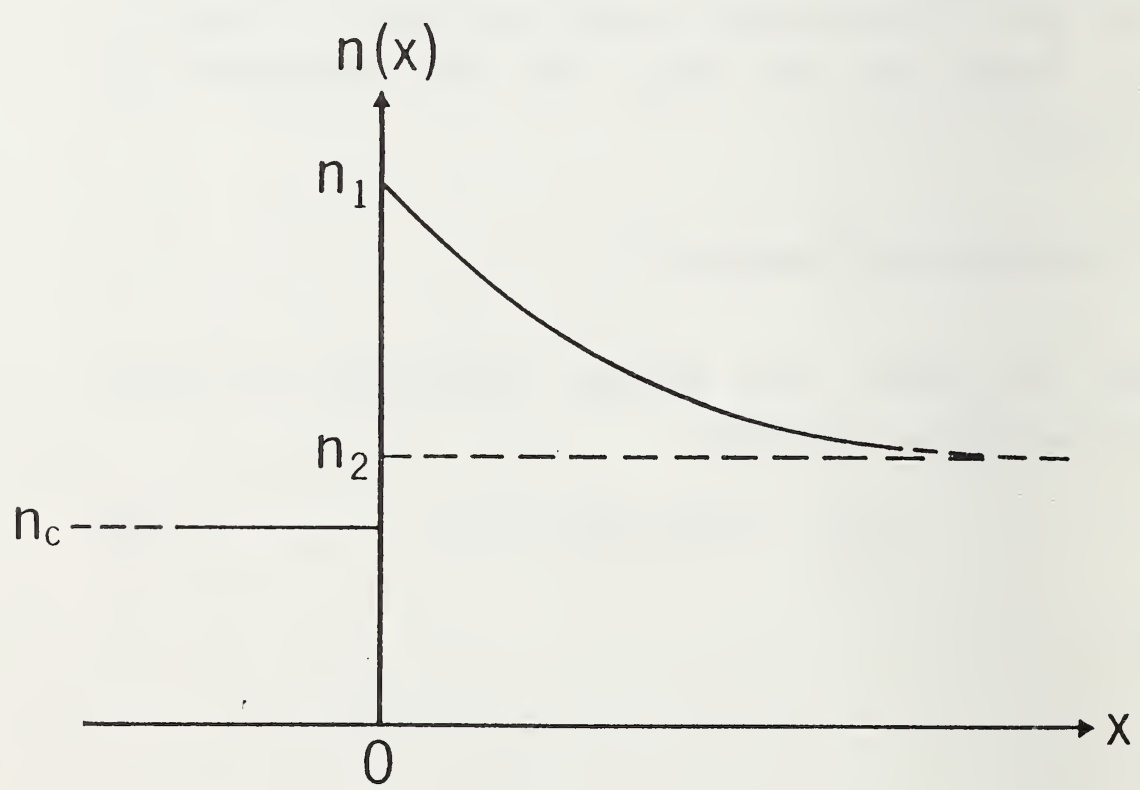

Figure 7.1 Refractive Index Profile for Example 7.1. 


$$
\begin{gathered}
\frac{d^{2} \psi}{d X^{2}}+V^{2}[\exp (-X)-b] \psi=0 \text { for } X>0, \\
\frac{d^{2} \psi}{d X^{2}}-V^{2}(b+B) \psi=0 \text { for } X<0,
\end{gathered}
$$

where

$$
\begin{gathered}
V^{2}=k_{0}^{2} d^{2}\left(n_{1}^{2}-n_{2}^{2}\right), \quad X=x / d, \\
B=\frac{n_{2}^{2}-n_{c}^{2}}{n_{1}^{2}-n_{2}^{2}}, \\
b=\frac{n_{e}^{2}-n_{2}^{2}}{n_{1}^{2}-n_{2}^{2}}, \text { and } n_{e}=\beta / k_{0} .
\end{gathered}
$$

The exact solution of Eqs. (3) was given in Example 6.1. It is repeated here for completeness.

$$
\Psi(X)= \begin{cases}\frac{J_{v}(2 V \exp (-X / 2))}{J_{v}(2 V)} & \text { for } X>0 \\ \exp (V \sqrt{b+B} X) & \text { for } X<0,\end{cases}
$$

where

$$
v=2 V \sqrt{b} .
$$

Equation (4a) satisfies the boundary condition for a guided mode; 
that is, $\Psi(X) \rightarrow 0$ as $X \rightarrow \pm \infty$. The multiplying constants have been chosen so that $\Psi(0)=1$. The continuity of $\Psi^{\prime}(X)$ at $X=0$ will yield the following eigenvalue equation for the normalized propagation constant $b$ :

$$
\frac{J_{v}^{\prime}(2 V)}{J_{v}(2 V)}=-\left(\frac{n_{e}^{2}-n_{c}^{2}}{n_{1}^{2}-n_{2}^{2}}\right)^{1 / 2} \text {. }
$$

The $M A F$ solution to Eq. (3a) is

$$
\Psi(X)=\left(\frac{\xi_{0}^{\prime}}{\xi^{\prime}}\right)^{1 / 2} \frac{A i(\xi)}{A i\left(\xi_{0}\right)} \text { for } X>0 .
$$

For $X<0$, the solution is the same as that given in Eq. (4a). $\xi(X)$ is given by

$$
\begin{aligned}
& \xi(x)=-[3 V(\sqrt{\exp (-X)-b} \\
& \left.\left.-\sqrt{b} \tan ^{-1} \sqrt{\frac{\exp (-X)}{b}-1}\right)\right]^{2 / 3} \text { for } X<X_{t}, \\
& \xi(x)=[3 V(-\sqrt{b-\exp (-X)} \\
& \left.\left.+\frac{\sqrt{b}}{2} \ln \frac{\sqrt{b}+\sqrt{b-\exp (-X)}}{\sqrt{b}-\sqrt{b-\exp (-X)}}\right)\right]^{2 / 3} \text { for } X>X_{t} .
\end{aligned}
$$


$X_{\mathrm{t}}$ is the turning point, that is, $b=\exp \left(-X_{\mathrm{t}}\right)$, and the subscript 0 indicates the value at $X=0$. Equation (6) satisfies the boundary condition $\Psi( \pm \infty) \rightarrow 0$ and the continuity condition $\Psi(0)=1$ at $X=0$.

The continuity of $\Psi^{\prime}(X)$ at $X=0$ yields the $M A F$ eigenvalue equation,

$$
\frac{V(B+b)^{1 / 2}}{\xi_{0}^{\prime}}=\frac{A i^{\prime}\left(\xi_{0}\right)}{A i\left(\xi_{0}\right)}-\frac{1}{2} \frac{\xi_{0}^{\prime \prime}}{\left(\xi_{0}^{\prime}\right)^{2}} .
$$

Equation (6) is an exact solution of the differential equation

$$
\begin{aligned}
& \frac{d^{2} \Psi}{d X^{2}}+V^{2}[\exp (-X)-b] \Psi \\
& +\left[\frac{1}{2} \frac{\xi^{\prime \prime \prime}}{\xi^{\prime}}-\frac{3}{4}\left(\frac{\xi^{\prime \prime}}{\xi^{\prime}}\right)^{2}\right] \Psi=0,
\end{aligned}
$$

as can be seen by substitution.

The $M A F$ solution will thus be a good approximation provided that the last term in Eq. (9) is small. This is so in practical cases, for which $\xi^{\prime \prime \prime}$ and $\xi$ "are small. This will be corroborated by the present example (cf. Eq. (2)). Comparing Eqs. (9) and (3a) and considering the last term in Eq. (9) as a perturbation, we get a first-order correction $\Delta b$ to the normalized propagation constant [Ghatak and Lokanathan (1984)] 


$$
\Delta b \cong \frac{-\int_{0}^{\infty}\left[\frac{1}{2} \frac{\xi^{\prime \prime \prime}}{\xi^{\prime}}-\frac{3}{4}\left(\frac{\xi^{\prime \prime}}{\xi^{\prime}}\right)^{2}\right] \psi^{2} d X}{V^{2} \int_{-\infty}^{\infty} \psi^{2}(X) d X} .
$$

To illustrate the accuracy of the method we have used the following values of the parameters in Eq. (2): $n_{2}=2.177$, $n_{\mathrm{c}}=1.0$, and $n_{1}^{2}-n_{2}^{2}=0.187$ [Ghatak, et. al. (1987), Gedeon (1974)]. The value of $d$ has been varied to obtain results as a function of $V$.

Table 7.1 gives the resulting $M A F$ values of $b$ as well as the exact values obtained from Eq. (5) and values obtained using the WKB method. The $M A F$ values are very good compared to $W K B$ for all values of $V$, but the correction given by Eq. (10) gives extremely accurate eigenvalues. The error is only about 0.03 percent even at $V=1.5$, that is, very near cutoff. The error $\Delta b$ in $b$ is related to error $\Delta \beta$ in $\beta$ by the relation [see Eq. (21) of Chapter 6],

$$
\frac{\Delta b}{b} \cong \frac{\Delta \beta}{\beta} \frac{n_{1}}{n_{1}-n_{2}} \frac{1}{b} \text {. }
$$

Thus, an error of 0.03 percent in $b$ in this case will correspond to an error of only about 0.00002 percent in $\beta$. 
Table 7.1

Normalized propagation constants for different values of $V$.

\begin{tabular}{||c|c|c|c|c||}
\hline \hline$V$ & Exact & $M A F$ & $\begin{array}{c}\text { MAF with } \\
\text { perturbation } \\
\text { Eq. (10) }\end{array}$ & WKB \\
\hline 1.5 & 0.03501 & 0.03609 & 0.03502 & 0.03783 \\
\hline 2.0 & 0.10495 & 0.10590 & 0.10503 & 0.10861 \\
\hline 2.5 & 0.17144 & 0.17216 & 0.17152 & 0.17531 \\
\hline 3.0 & 0.22919 & 0.22974 & 0.22926 & 0.23308 \\
\hline 3.5 & 0.27865 & 0.27908 & 0.27872 & 0.28249 \\
\hline 4.0 & 0.32118 & 0.32152 & 0.32124 & 0.32493 \\
\hline 5.0 & 0.39029 & 0.39052 & 0.39037 & 0.39385 \\
\hline 6.0 & 0.44408 & 0.44424 & 0.44411 & 0.44744 \\
\hline 7.0 & 0.48724 & 0.48737 & 0.48727 & 0.49043 \\
\hline 8.0 & 0.52278 & 0.52287 & 0.52280 & 0.52580 \\
\hline
\end{tabular}

Example 7.2

We next revisit Example 6.5 of Chapter 6. We consider Schrödinger's equation for a spherically symmetric potential corresponding to $\ell=0$. We saw in Example 6.5 that the eigenvalues can be determined from the tabulated zeros of the Airy 
function and, in the process, we noted that we should expect that accuracy of the $M A F$ eigenvalues should be of the same order as the WKB eigenvalues; however, the first-order perturbation theory (discussed in the preceding example) allows us to obtain very good accuracy for the eigenvalues.

For a spherically symmetric potential $V(r)$ the radial part of the wave function $R(r)$ satisfies the equation

$$
\begin{gathered}
\frac{1}{r^{2}} \frac{d}{d r}\left[r^{2} \frac{d R}{d r}\right]+\frac{2 \mu}{\hbar^{2}}[E-V(r)- \\
\left.\frac{\ell(\ell+1) \hbar^{2}}{2 \mu r^{2}}\right] R(r)=0,
\end{gathered}
$$

where $E$ is the energy eigenvalue and the other symbols have their usual meaning. Following the analysis given in Example 6.5, we find that for $\ell=0, \Psi(r)$ given by Eq. (82) of Chapter 6 is an exact solution of the differential equation

$$
\frac{d^{2} \psi}{d r^{2}}+\left[\Gamma^{2}(r)+F(r)\right] \psi(r)=0,
$$

where

$$
F(r)=\frac{1}{2} \frac{\xi^{\prime \prime \prime}}{\xi^{\prime}}-\frac{3}{4}\left(\frac{\xi^{\prime \prime}}{\xi^{\prime}}\right)^{2},
$$

$\Psi(\mathrm{r})=r R(r)$ and $\Gamma^{2}(r)$ is given by Eq. (81) of Chapter 6 . Because of the last term in Eq. (13) is a perturbation term, we get 


$$
\Delta E=-\frac{\hbar^{2}}{2 \mu} \frac{\int_{0}^{\infty} F(r) \Psi^{2}(r) d r}{\int_{0}^{\infty} \Psi^{2}(r) d r}
$$

for the first-order correction $\Delta E$ to the energy eigenvalue.

As in Chapter 6, we use the profile

$$
V(r)=-V_{0} \exp \left(-\frac{r}{a}\right) .
$$

The exact eigenvalues are obtained by solving the transcendental equation

$$
J_{v}(g)=0
$$

[See Eqs. (93) and (94) of Chapter 6.] For the deuteron problem (cf. Example 6.5, Chapter 6),

$$
g \propto 3.64
$$

Equation (17) yields $v=0.858$ 628. The corresponding $M A F$ value of $v$ obtained from Eq. (89) of Chapter 6 is 0.865815 . The perturbation correction [see Eq. (15)] yields $v=0.85873$, which agrees extremely well with the exact result. The corresponding $W K B$ value is 0.886341 .

For this example, then, accuracy of the eigenvalue using the $M A F$ method is considerably higher than that of the WKB method. In this regard, see Table 7.2, where the error is 3.2 percent (WKB) versus 0.84 percent. The value of the eigenvalue obtained when 
using the correction given by Eq. (15) is very impressive. The error is only 0.012 percent for this example.

\begin{tabular}{||c|c|c|c||}
\hline \multicolumn{4}{|c||}{ Table 7.2} \\
\hline$v_{\text {EXACT }}$ & $\frac{v_{\text {MAF }}}{v_{\text {EXACT }}}$ & $\frac{v_{\text {PERTURBATION }}}{v_{\text {EXACT }}}$ & $\frac{v_{\text {WKB }}}{v_{\text {EXACT }}}$ \\
\hline 0.858628 & 1.0084 & 1.00012 & 1.032 \\
\hline
\end{tabular}

For $g=3.64$, there is only one bound state. In general, if there is more than one bound state the MAF solutions will be more accurate for higher-order states.

\section{Example 7.3}

In this example we use the $M A F$ method together with the firstorder perturbations theory to evaluate the energy eigenvalues for an anharmonic oscillator, characterized by the following potential

$$
V(x)=\frac{1}{2} k x^{2}+a x^{4}, k>0 \text { and } a>0 .
$$

This potential is important in many physical problems. Very accurate eigenvalues for this potential have been obtained by Hioe and Montroll (1975). Later, Kesarwani and Varshni (1981) used 
the five-term (eighth order) WKB approximation to calculate the eigenvalues and compared them with those obtained by Hioe and Montrol (HM).

We start with the Schrödinger equation

$$
\frac{d^{2} \psi}{d x^{2}}+\frac{2 m}{\hbar^{2}}[E-V(x)] \Psi=0,
$$

where the symbols have their usual meanings. For the potential given by Eq. (18), the Schrödinger equation can be transformed to the following form,

$$
\frac{d^{2} \psi}{d X^{2}}+\Gamma^{2}(X) \psi(X)=0,
$$

where

$$
\begin{gathered}
\Gamma^{2}(X)=\frac{2}{\lambda^{2}}\left(X^{2}+\beta_{1}\right)\left(\beta_{2}-X^{2}\right), \\
X=\sqrt{\frac{a}{k}} x,
\end{gathered}
$$




$$
\begin{gathered}
\lambda=\frac{a \hbar}{\sqrt{m k^{3}}}, \\
\beta_{1}=\frac{1}{4}\left[1+\frac{1}{1-2 \omega^{2}}\right], \\
\beta_{2}=\frac{1}{4}\left[-1+\frac{1}{1-2 \omega^{2}}\right], \\
\omega=\left[\frac{1}{2}-\frac{1}{2}\left(1+\frac{16 a E}{k^{2}}\right)^{-1 / 2}\right]^{1 / 2} .
\end{gathered}
$$

In the same units as those used by Hioe and Montroll, the eigenvalue for the state with quantum number $n$ is given by

$$
E_{n}=\left[\frac{1}{\left(1-2 \omega^{2}\right)^{2}}-1\right] / 16 \lambda,
$$

where $\omega^{2}$ is determined by solving Eq. (20).

The MAF solution of Eq. (20) for a bound mode is given by 


$$
\Psi(X)=\frac{A i[\xi(X)]}{\sqrt{\xi^{\prime}(X)}}
$$

where

$$
\begin{gathered}
\xi(X)=\left[\frac{3}{2} \int_{X}^{\sqrt{\beta_{2}}} \sqrt{-\Gamma^{2}(X)} d X\right]^{2 / 3} ; X>0 \\
=-\left\{\frac{3}{2} \int_{X}^{\sqrt{\beta_{2}}} \frac{\sqrt{2}}{\lambda}\left[\left(X^{2}+\beta_{1}\right)\left(\beta_{2}-X^{2}\right)\right]^{1 / 2} d X\right\}^{2 / 3} ; \\
0<X<\sqrt{\beta_{2}},
\end{gathered}
$$

or

$$
\begin{gathered}
\xi(X)=\left\{\frac{3}{2} \int_{\sqrt{\beta_{2}}}^{x} \frac{\sqrt{2}}{\lambda}\left[\left(X^{2}+\beta_{1}\right)\left(X^{2}-\beta_{2}\right)\right]^{1 / 2} d X\right\}^{2 / 3} ; \\
X>\sqrt{\beta_{2}},
\end{gathered}
$$

In writing Eq. (22), we used the fact that for a bound mode $\Psi(x) \rightarrow 0$ as $x \rightarrow \pm \infty$, hence the omission of the term proportional to $B i(x)$. The solution given by Eq. (22) is arbitrary to the extent of a multiplicative constant. 
Since the potential given by Eq. (18) is symmetric, we will have symmetric and antisymmetric modes. For brevity, we consider only the antisymmetric modes, for which $\Psi(0)=0$ and, therefore,

$$
\operatorname{Ai}[\xi(0)]=0 .
$$

Following Example 6.2, the above equation readily leads to

$$
\int_{0}^{\sqrt{\beta_{2}}} \Gamma(x) d x=\left(\xi_{a n}+\frac{1}{2}\right) \frac{\pi}{2},
$$

where $\zeta_{\text {an }}$ are given in Table 6.2. The corresponding first-order $W K B$ quantization condition is given by

$$
\int_{0}^{\sqrt{\beta_{2}}} \Gamma(x) d x=\left(m+\frac{1}{2}\right) \frac{\pi}{2} \quad m=1,3,5, \ldots .
$$

It is obvious from Eqs. (26) and (27) that the numerical effort required to calculate the eigenvalues by the $M A F$ method is the same as that required by using the first-order WKB method. Following Examples 7.1 and 7.2, the first-order perturbation correction to $E_{\mathrm{n}}$ is given by 


$$
\Delta E=\frac{\frac{\lambda}{2} \int_{0}^{\infty}\left(\frac{1}{2} \frac{\xi^{\prime \prime \prime}}{\xi^{\prime}}-\frac{3}{4}\left(\frac{\xi^{\prime \prime}}{\xi^{\prime}}\right)^{2}\right) \psi^{2}(X) d X}{\int_{0}^{\infty} \Psi^{2}(X) d X},
$$

where primes denote differentiation with respect to $X$. Examination of the five-term (eighth order) WKB formulas for determining the eigenvalues [see Kesarwani and Varshni (1981)] illustrates the strength of the $M A F$ method. Clearly, the $M A F$ results (with perturbation correction) are much easier to use than the much more complicated five-term WKB expansion.

Using the $M A F$ method, we have calculated the eigenvalues $E_{\mathrm{n}}$ for $n=1, \lambda=0.002,1,2$, and 50. The results are given in Table 7.3, column 4 . We have also given the eigenvalues calculated using the first-order (column 3 of Table 7.3) and the five-term (eighth-order) $W K B$ method, taken from Kesarvani and Varshni (1981) and labeled KV in the table. The values given by Hioe and Montroll (1975) are also reproduced in the table and are labeled HM. It is these results (HM) against which comparison will be made.

Except for $\lambda=0.002$, the $M A F$ results are more accurate than the first-order $W K B$ results. The accuracy of $W K B$ results at $\lambda=0.002$ is due to the fact that $\lambda=0$ corresponds to the case of a simple harmonic oscillator for which the $W K B$ method yields exact results. Though in comparison to the $M A F$ results, the five- 
term $W K B$ results are in better agreement with the $\mathrm{HM}$ results, the numerical effort involved in the latter is much more. We have also found the first-order perturbation correction to the $M A F$ eigenvalues. With this correction, the $M A F$ eigenvalues agree better with HM results than the five-term $W K B$ results.

\begin{tabular}{|c|c|c|c|c|c|}
\hline \multicolumn{6}{|c|}{$\begin{array}{l}\text { Energy eigenvalues for the anharmonic oscillator, } \\
\qquad n=1\end{array}$} \\
\hline$\lambda$ & $\mathrm{E}^{\mathrm{WKB}}$ & $\mathrm{E}^{\mathrm{MAF}}$ & $\mathrm{E}^{\mathrm{KV}}$ & $\mathrm{E}^{\mathrm{HM}}$ & $\mathrm{E}^{\mathrm{MAF}+\mathrm{PERT}}$ \\
\hline 0.002 & 1.5067397 & 1.52419859 & 1.50741939 & 1.50741939 & 1.50743212 \\
\hline 1 & 2.70348341 & 2.74270374 & 2.73974461 & 2.73789227 & 2.73798564 \\
\hline 2 & 3.25095901 & 3.29911586 & 3.29637221 & 3.29286782 & 3.29302536 \\
\hline 50 & 8.80254714 & 8.93782122 & 8.93357114 & 8.91509636 & 8.91574779 \\
\hline
\end{tabular}

\subsection{Conclusions}

In summary, the $M A F$ method not only allows an accurate description of the wave function, but of the eigenvalues as well.

The results given in the tables are extremely good. The $M A F$ method in itself gives good agreement with the exact eigenvalues. The correction introduced in this chapter, while mildly complicated, gives results that are very nearly equal to the exact values. When good accuracy is required, the method given here 
is viable.

We saw in Chapter 6 that, in some cases, there is a clear $M A F$ analog to the $W K B$ quantization condition, suggesting that the accuracy of the $M A F$ method will be about the same as the WKB method. In fact, the $M A F$ eigenvalues, without the perturbation improvement, are considerably more accurate than the WKB method. 



\section{REFERENCES}

Abramowitz, M., and Stegun, I.A. (1970), Handbook of Mathematical Functions with Formulas, Graphs and Mathematical Tables, U.S. Department of Commerce, National Bureau of Standards, Washington, D.C., Applied Mathematical Series 55, Chapter 10.

Bahar, E. (1967), Generalized WKB method with applications to problems of propagation in nonhomogeneous media, J. Math. Phys., Vol. 8, 1735-1746.

Bender, Carl M., and Orszag, S.A. (1978), Advanced Mathematical Methods for Scientists and Engineers, McGraw-Hill Book Company, New York, Chapter 10. See also Felsen, L.B., and Marcuvitz, N. (1973), Radiation and Scattering of Waves, Prentice-Hall, Inc., New York, Section 3.5, or Jones, D.S. (1964), The Theory of Electromagnetism, The Macmillan Company, New York, Section 6.22.

Brillouin, L. (1926), The undulatory mechanics of Schrödinger, Compt. Rend. Acad. Sci. Paris, Vol. 183, 24-26.

Conwell, E.M. (1973), Modes in optical waveguides formed by diffusion, Appl. Phys. Lett., Vol. 23, 328-330. 
Fröman, N., and Fröman, P.O. (1965), JWKB Approximation: Contributions to the Theory, North Holland Publishing Company, Amsterdam.

Gedeon, A. (1974), Comparison between rigorous theory and WKB analysis of modes in graded index waveguides, Opt. Commun., Vol. 12, 329-332.

Ghatak, A.K., and Lokanathan, S. (1984), Quantum Mechanics: Theory and Applications, Macmillan Press, New Delhi.

Ghatak, A. K., and Thyagarajan, K. (1989), Optical Electronics, Cambridge University Press, Cambridge, UK.

Ghatak, A.K., Thyagarajan, K., and Shenoy, M.R. (1987), Numerical analysis of planar optical waveguides using matrix approach, IEEE J. Lightwave Tech., Vol. LT-5, 660-667.

Ghatak, A.K., Gallawa, R.L., and Goyal, I.C. (1991), Accurate solutions to Schrödinger's equation using modified Airy functions, submitted for publication.

Ginzburg, V.L. (1964), The Propagation of Electromagnetic Waves in Plasmas, Pergamon Press, New York.

Goyal, I.C., Gallawa, R.L., and Ghatak, A.K. (1991a), An approximate solution to the wave equation-revisited, J. Electromagnetic Waves and Appl., Vol. 5, 623-636. 
Goyal, I.C., Gallawa, R.L., and Ghatak, A.K. (1991b), Approximate solution to the scalar wave equation for planar optical waveguides, Appl. Opt., Vol. 30, 2985-2990.

Goyal, I.C., Gallawa, R.L., and Ghatak, A.K. (1991c), Methods of analyzing planar optical waveguides, Opt. Lett., Vol. 16, 3032.

Heading, J. (1962), An Introduction to Phase Integral Methods, Methuen and Co., London.

Hecht, C.E., and Mayer, J.E. (1957), Extension of the WKB equation, Phys. Rev., Vol. 106, 1156-1160.

Hioe, F.T., and Montroll, E.W. (1975), Quantum theory of anharmonic oscillators, I. Energy levels of oscillators with positive quartic anharmonicity, J. Math. Physics, Vol. 16, 1945-1955.

Irving, J., and Mullineux, N. (1959), Mathematics in Physics and Engineering, Academic Press, New York.

Jeffreys, H. (1923), On certain approximate solutions of linear differential equations of second order, Proc. London Math. Soc., Vol. 23, No. 2, 428-436.

Kesarwani, R.N., and Varshni, Y.P. (1981), Eigenvalues of an anharmonic oscillator, J. Math. Physics, Vol. 22, 1983-1989. 
Kramers, H.A. (1926), Wellenmechanik und halbzahlige quantisierung, Zeits. f. Physik, Vol. 39, 828-840.

Langer, R.E. (1931), On the asymptotic solutions of ordinary differential equations, with an application to the Bessel functions of large order, Trans. Am. Math. Soc., Vol. 33, 23-64.

Magnus, W., Oberhettinger, F., and Soni, R.P. (1966), Formulas and Theorems for the Special Functions of Mathematical Physics, Springer-Verlag, Berlin.

Pauli, W. (1980) General Principles of Quantum Mechanics, in English, translated from German by P. Achuthan and K. Venkatesan, Allied Publishers Private Ltd., Bombay.

Schiff, L.I. (1968), Quantum Mechanics, McGraw-Hill Book Co., New York.

Wentzel, Gregor (1926), Eine Verallgemeinerung der quantenbedingung für die zweeke der wellenmechanik, Zeits. f. Physik, Vol. 38, 518-529. 


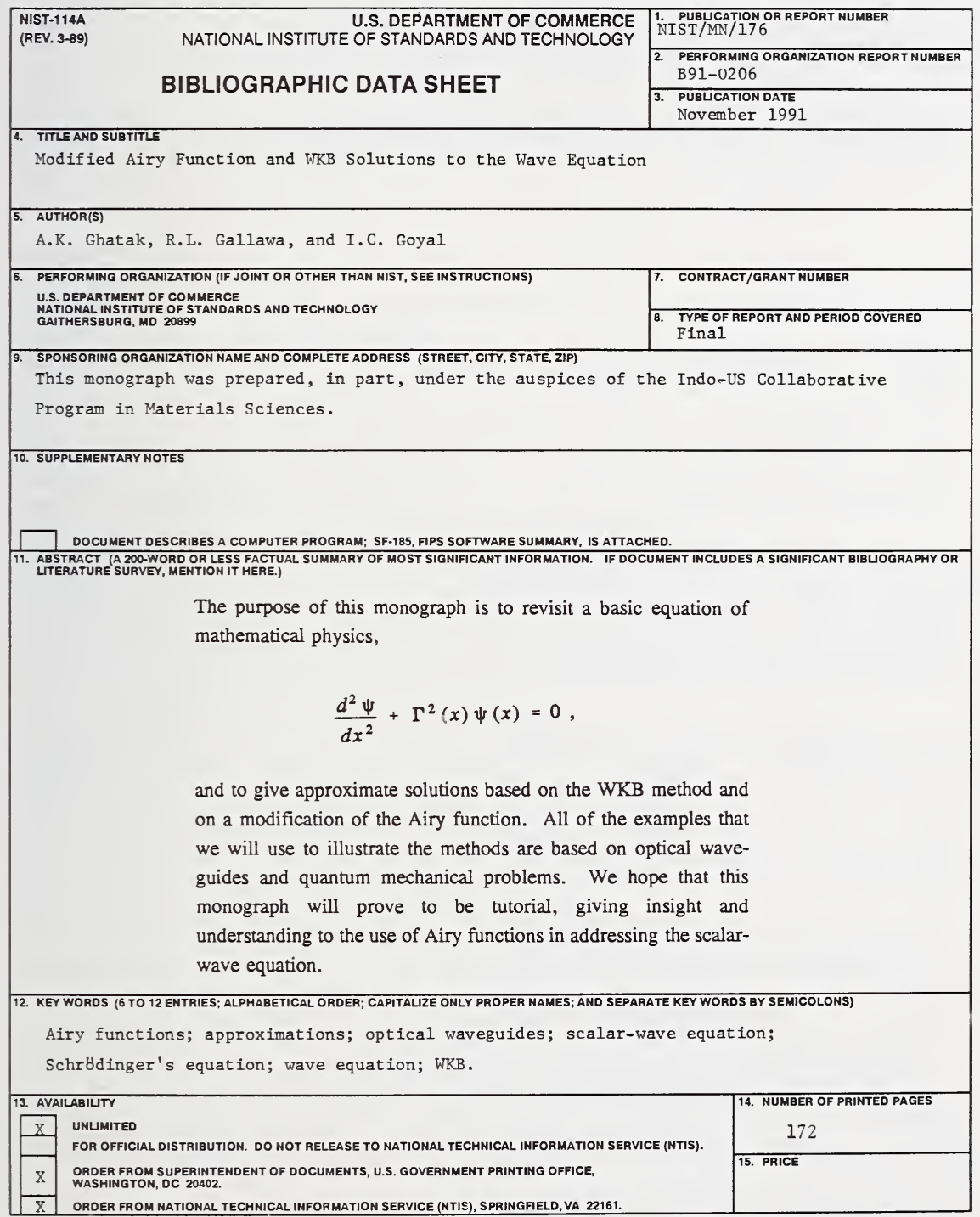

ELECTRONIC FORM 



\section{NTSTTechnical Publications}

\section{Periodical}

Journal of Research of the National Institute of Standards and Technology - Reports NIST research and development in those disciplines of the physical and engineering sciences in which the Institute is active. These include physics, chemistry, engineering, mathematics, and computer sciences.

Papers cover a broad range of subjects, with major emphasis on measurement methodology and the basic technology underlying standardization. Also included from time to time are survey articles on topics closely related to the Institute's technical and scientific programs. Issued six times a year.

\section{Nonperiodicals}

Monographs-Major contributions to the technical literature on various subjects related to the Institute's scientific and technical activities.

Handbooks-Recommended codes of engineering and industrial practice (including safety codes) developed in cooperation with interested industries, professional organizations, and regulatory bodies.

Special Publications - Include proceedings of conferences sponsored by NIST, NIST annual reports, and other special publications appropriate to this grouping such as wall charts, pocket cards, and bibliographies.

Applied Mathematics Series-Mathematical tables, manuals, and studies of special interest to physicists, engineers, chemists, biologists, mathematicians, computer programmers, and others engaged in scientific and technical work.

National Standard Reference Data Series-Provides quantitative data on the physical and chemical properties of materials, compiled from the world's literature and critically evaluated. Developed under a worldwide program coordinated by NIST under the authority of the National Standard Data Act (Public Law 90-396). NOTE: The Journal of Physical and Chemical Reference Data (JPCRD) is published bi-monthly for NIST by the American Chemical Society (ACS) and the American Institute of Physics (AIP). Subscriptions, reprints, and supplements are available from ACS, 1155 Sixteenth St., NW., Washington, DC 20056.

Building Science Series - Disseminates technical information developed at the Institute on building materials, components, systems, and whole structures. The series presents research results, test methods, and performance criteria related to the structural and environmental functions and the durability and safety characteristics of building elements and systems.

Technical Notes-Studies or reports which are complete in themselves but restrictive in their treatment of a subject. Analogous to monographs but not so comprehensive in scope or definitive in treatment of the subject area. Often serve as a vehicle for final reports of work performed at NIST under the sponsorship of other government agencies.

Voluntary Product Standards - Developed under procedures published by the Department of Commerce in Part 10, Title 15, of the Code of Federal Regulations. The standards establish nationally recognized requirements for products, and provide all concerned interests with a basis for common understanding of the characteristics of the products. NIST administers this program as a supplement to the activities of the private sector standardizing organizations.

Consumer Information Series - Practical information, based on NIST research and experience, covering areas of interest to the consumer. Easily understandable language and illustrations provide useful background knowledge for shopping in today's technological marketplace.

Order the above NIST publications from: Superintendent of Documents, Government Printing Office, Washington, DC 20402.

Order the following NIST publications-FIPS and NISTIRs-from the National Technical Information Service, Springfield, VA 22161.

Federal Information Processing Standards Publications (FIPS PUB) - Publications in this series collectively constitute the Federal Information Processing Standards Register. The Register serves as the official source of information in the Federal Government regarding standards issued by NIST pursuant to the Federal Property and Administrative Services Act of 1949 as amended, Public Law 89-306 (79 Stat. 1127), and as implemented by Executive Order 11717 (38 FR 12315, dated May 11, 1973) and Part 6 of Title 15 CFR (Code of Federal Regulations).

NIST Interagency Reports (NISTIR) - A special series of interim or final reports on work performed by NIST for outside sponsors (both government and non-government). In general, initial distribution is handled by the sponsor; public distribution is by the National Technical Information Service, Springfield, VA 22161, in paper copy or microfiche form. 



